\title{
Radially $\pi$-Extended Pyrrole-Fused Azacoronene: A Series of Crystal Structures of HPHAC with Various Oxidation States
}

Yoshiki Sasaki, ${ }^{[a]}$ Masayoshi Takase, ${ }^{*[a]}$ Nagao Kobayashi, ${ }^{*}{ }^{[b]}$ Shigeki Mori, ${ }^{[c]}$ Keishi Ohara, ${ }^{[a]}$ Tetsuo Okujima, ${ }^{[a]}$ and Hidemitsu Uno ${ }^{*}[\mathrm{a}]$

${ }^{[a]}$ Graduate School of Science and Engineering, Ehime University, Matsuyama 790-8577, Japan

${ }^{[b]}$ Faculty of Textile Science and Technology, Shinshu University, Ueda 386-8567, Japan

${ }^{[c]}$ Advanced Research Support Center (ADRES), Ehime University, Matsuyama 790-8577, Japan

E-mail: takase.masayoshi.ry@ehime-u.ac.jp

E-mail: nagaok@shinshu-u.ac.jp

E-mail: uno@ehime-u.ac.jp

\section{Contents}

1. ${ }^{1} \mathrm{H}$ - and ${ }^{13} \mathrm{C}\left\{{ }^{1} \mathrm{H}\right\}$-NMR spectra of $\mathbf{4}$ and $\mathbf{2} \ldots \quad$ p. S2

2. Comparison of ${ }^{1} \mathrm{H}-\mathrm{NMR}$ spectra of $\mathbf{4}, \mathbf{2}$, and $\mathbf{2}^{2+} \ldots \quad$ p. S4

3. X-ray crystal structures of $\mathbf{4}, \mathbf{2}, \mathbf{2}^{++}$, and $\mathbf{2}^{2+} \ldots \quad$ p. S5

$\begin{array}{ll}\text { 4. Comparison of bowl depths of } \mathbf{2}, \mathbf{2}^{\mathbf{}} \text {, and } \mathbf{2}^{2+} \ldots & \text { p. S12 }\end{array}$

5. Intermolecular interaction of $\mathbf{2}$ in the packing structure ... p. S13

$\begin{array}{ll}\text { 6. Molecular orbital diagrams of 1a and 2-H ... } & \text { p. S14 }\end{array}$

$\begin{array}{ll}\text { 7. Cyclic voltammetry of } 2 \ldots & \text { p. S16 }\end{array}$

$\begin{array}{ll}\text { 8. Stepwise oxidation of } \mathbf{2} \text { with } \mathrm{SbCl}_{5} \ldots & \text { p. S17 }\end{array}$

9. ESR spectra of $\mathbf{2}^{++}$and $\mathbf{2}^{2+}$ and spin density map of $\mathbf{2}-\mathbf{H}^{++} \ldots \quad$ p. S18

10. NICS values of $\mathbf{1 b}$ and $\mathbf{2}-\mathbf{H}$, and $\mathbf{2}-\mathbf{H}^{2+} \ldots \quad$ p. S19

11. TD-DFT calculations of $\mathbf{2}-\mathbf{H}, \mathbf{2}-\mathbf{H}^{++}$, and $\mathbf{2}-\mathbf{H}^{2+} \ldots \quad$ p. S21

$\begin{array}{ll}\text { 12. Titration experiments of } \mathbf{2} \text { with } \mathrm{C}_{60} \ldots & \text { p. S28 }\end{array}$

$\begin{array}{ll}\text { 13. MCD spectra of 1a and } 2 \ldots & \text { p. S32 }\end{array}$

14. Atomic coordinates of $\mathbf{2}-\mathbf{H}, \mathbf{2}-\mathbf{H}^{++}$, and $\mathbf{2}-\mathbf{H}^{2+} \ldots \quad$ p. S35

$\begin{array}{ll}\text { 15. References ... } & \text { p. S39 }\end{array}$ 
1. ${ }^{1} \mathrm{H}$ - and ${ }^{13} \mathrm{C}\left\{{ }^{1} \mathrm{H}\right\}$-NMR spectra of 4 and 2

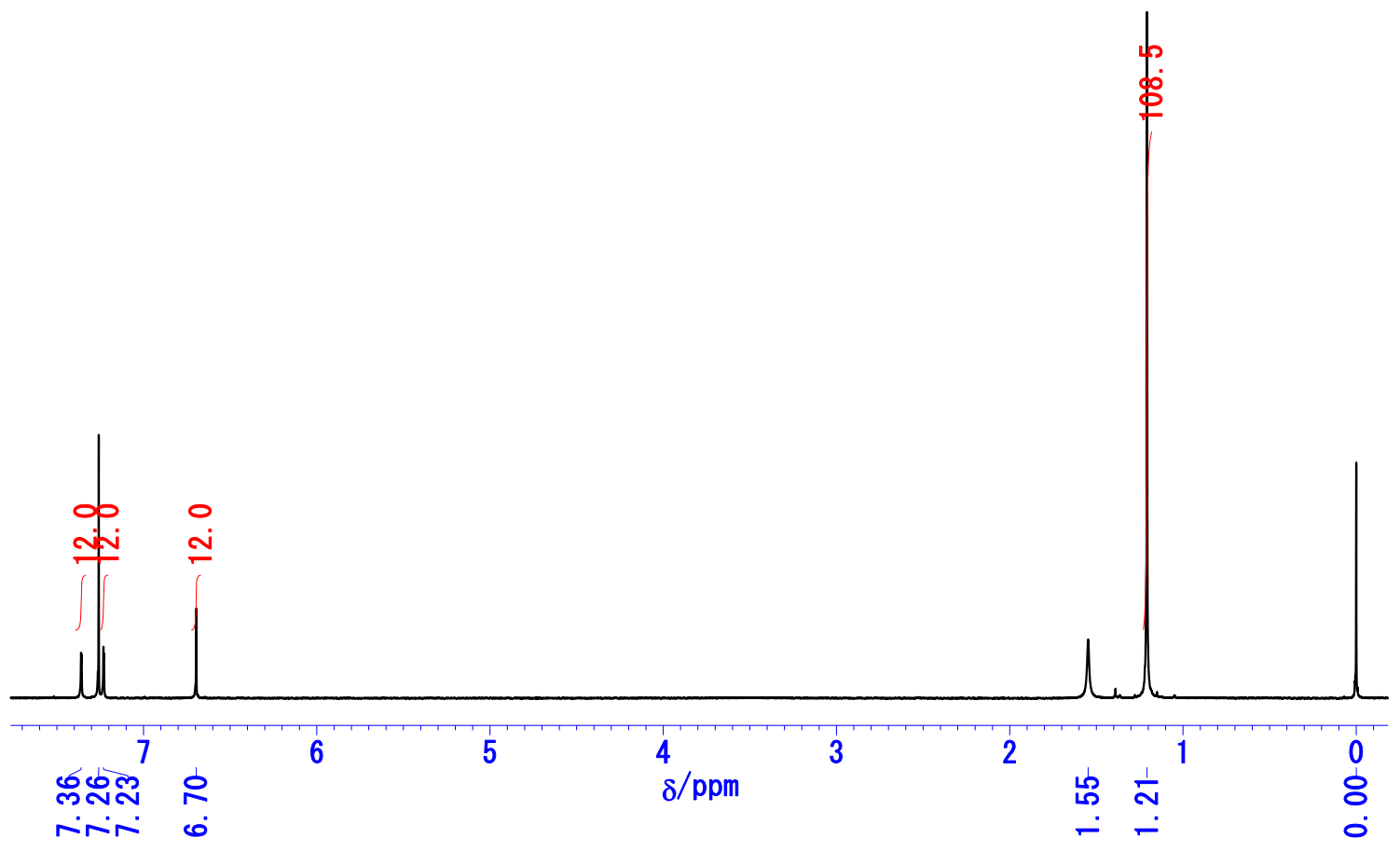

Figure 1a. ${ }^{1} \mathrm{H}-\mathrm{NMR}$ spectrum of 4 in $\mathrm{CDCl}_{3}(400 \mathrm{MHz})$.

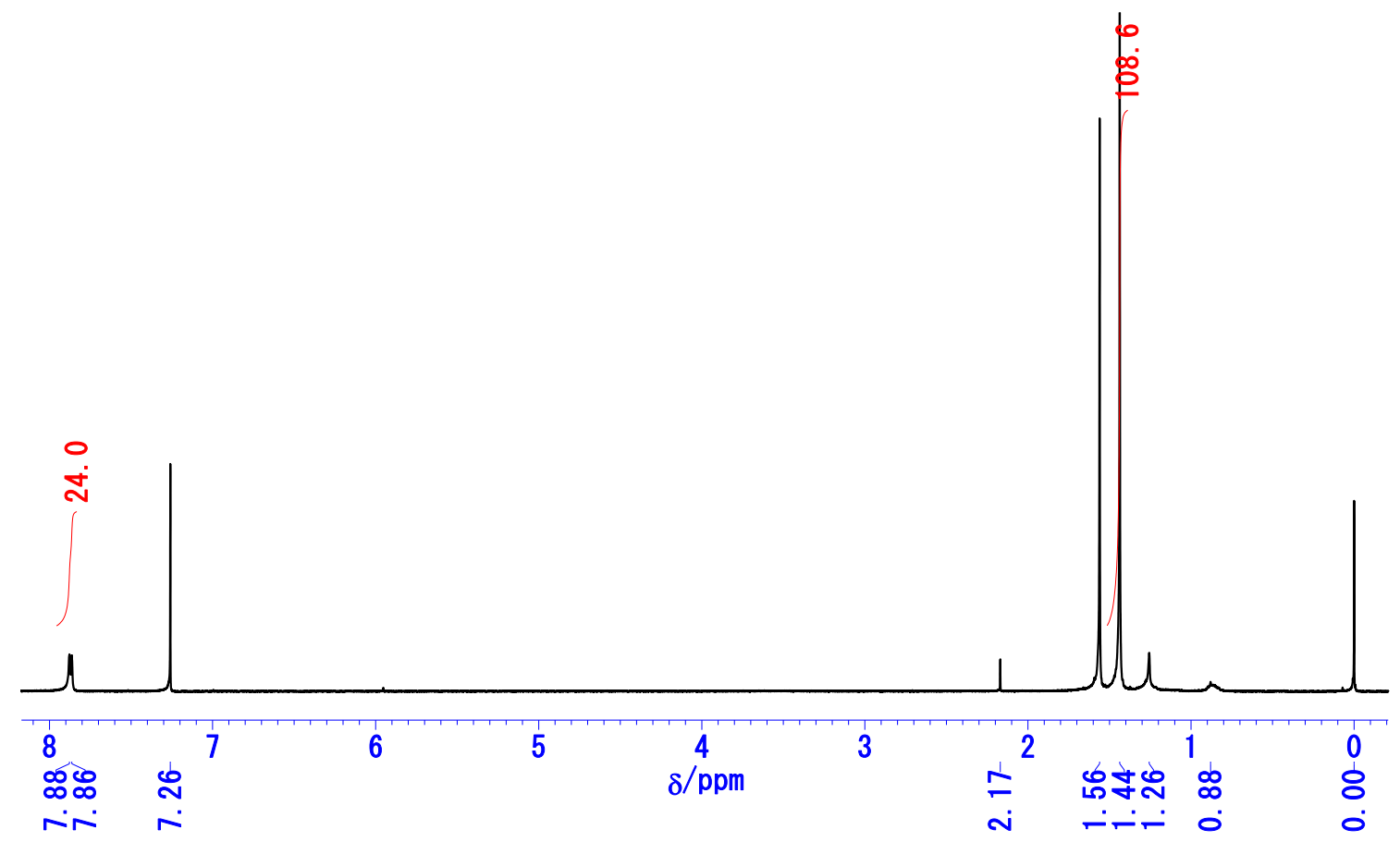

Figure 1b. ${ }^{1} \mathrm{H}-\mathrm{NMR}$ spectrum of $\mathbf{2}$ in $\mathrm{CDCl}_{3}(400 \mathrm{MHz})$. 


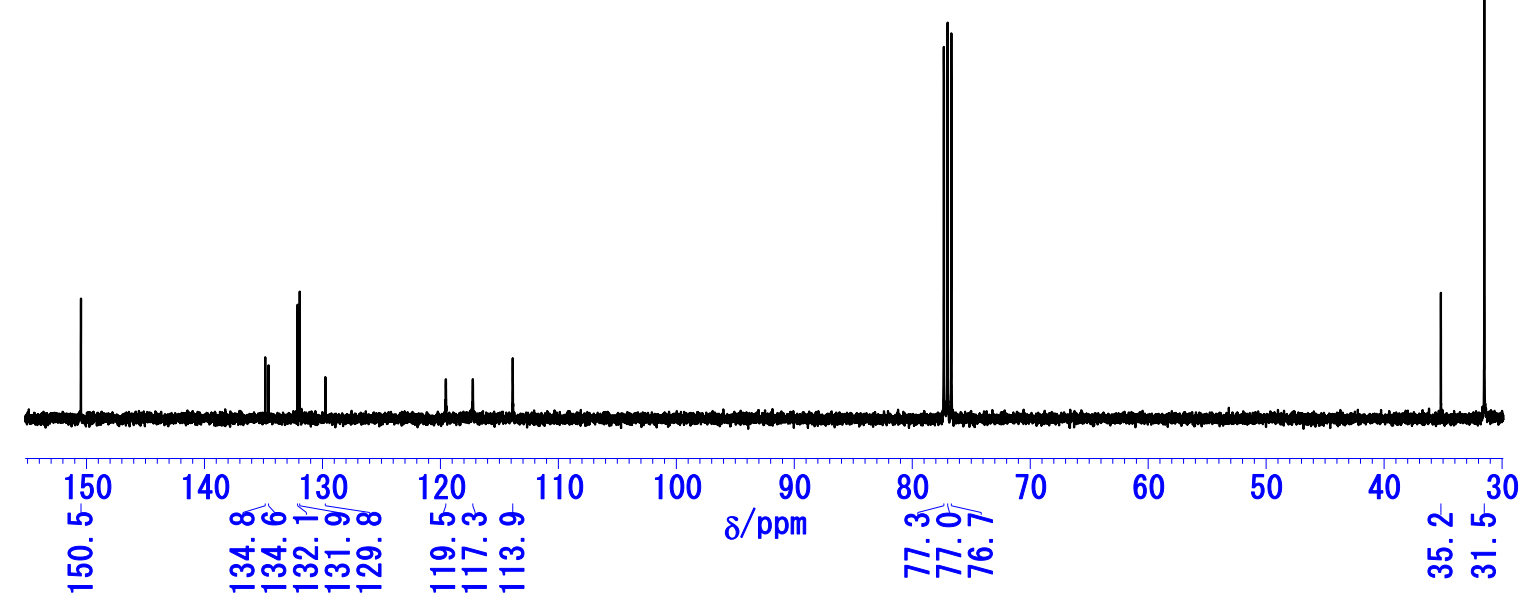

Figure 1c. ${ }^{13} \mathrm{C}\left\{{ }^{1} \mathrm{H}\right\}$-NMR spectrum of 4 in $\mathrm{CDCl}_{3}(100 \mathrm{MHz})$.

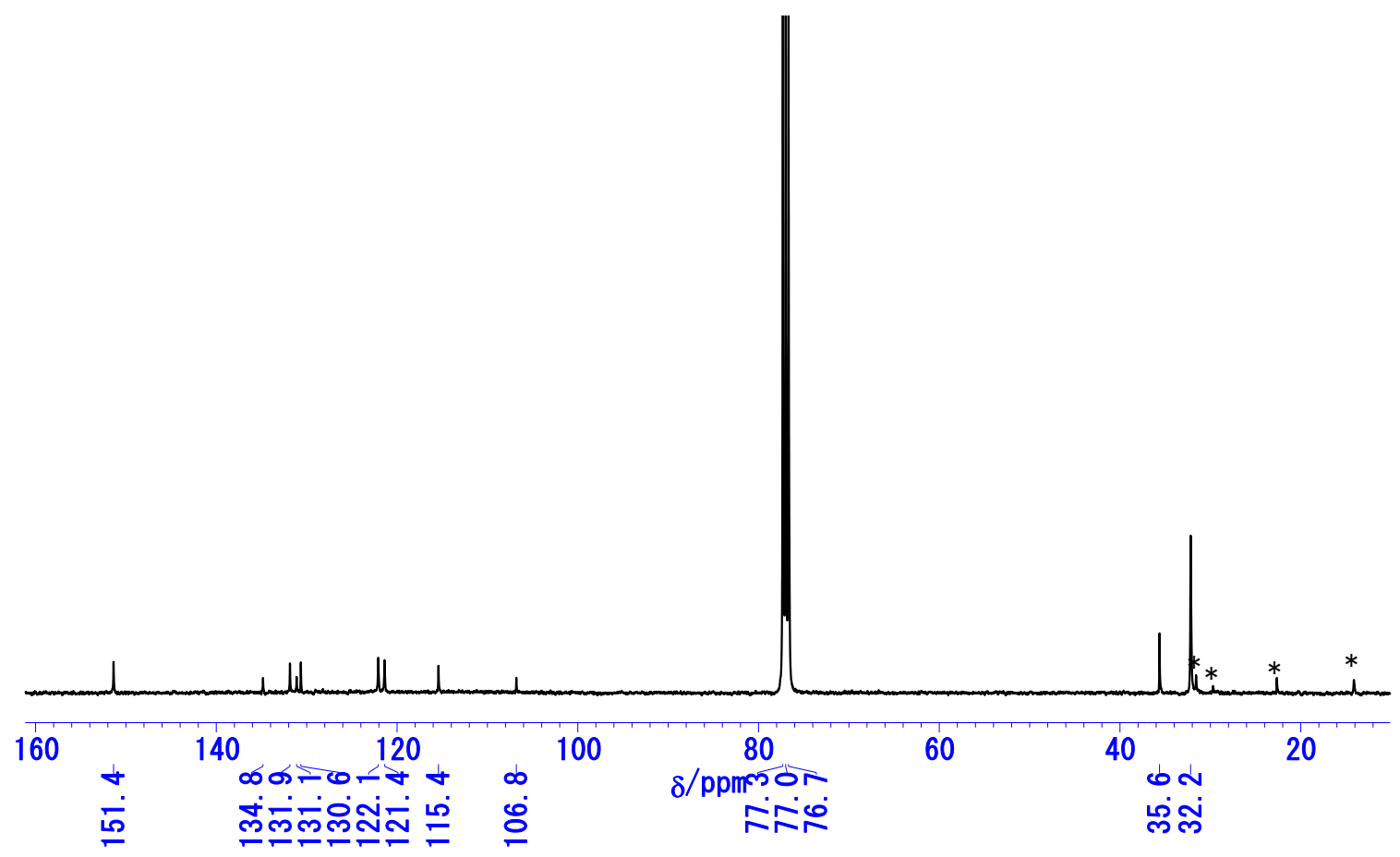

Figure 1d. ${ }^{13} \mathrm{C}\left\{{ }^{1} \mathrm{H}\right\}$-NMR spectrum of $\mathbf{2}$ in $\mathrm{CDCl}_{3}(100 \mathrm{MHz})$ (*: impurity peak). 
2. Comparison of ${ }^{1} \mathrm{H}-\mathrm{NMR}$ spectra of 4,2 , and $2^{2+}$

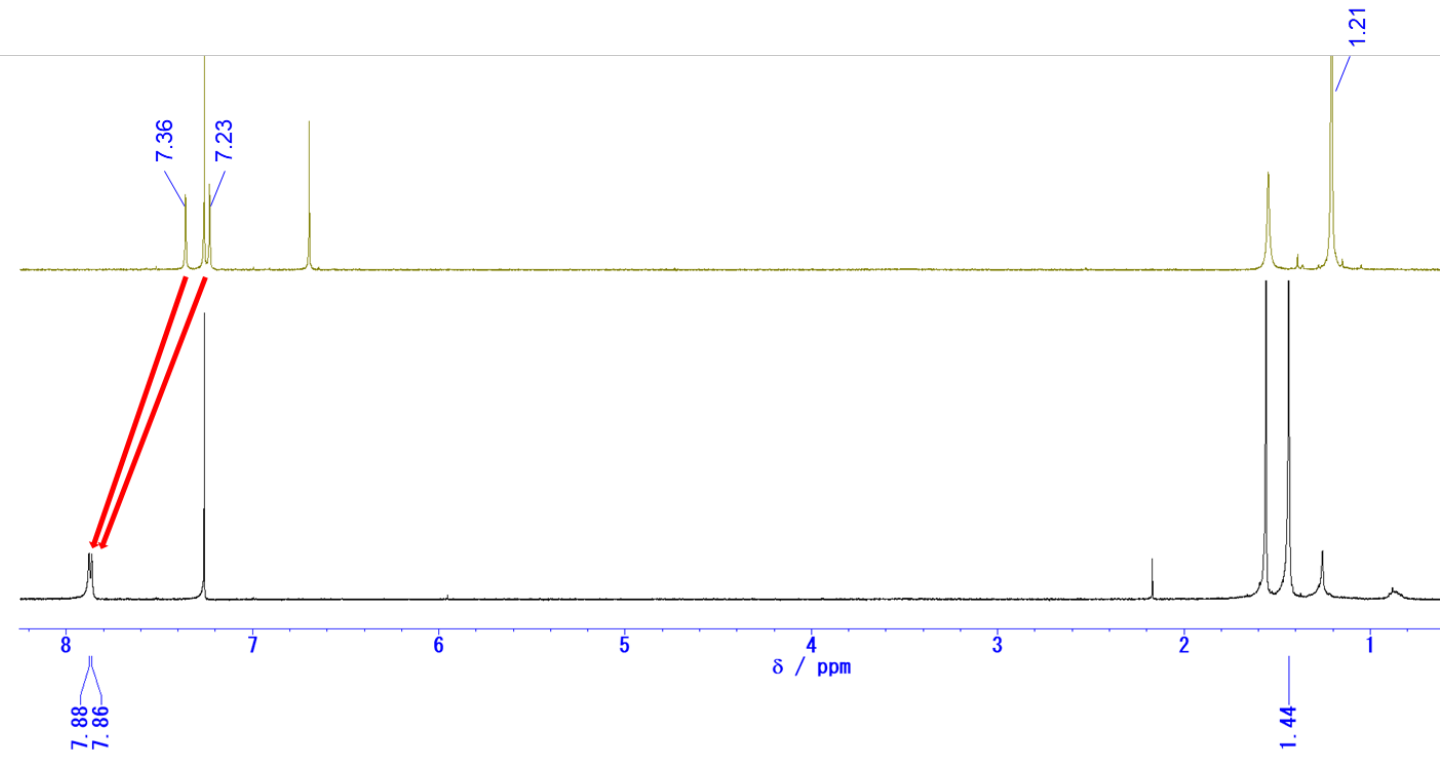

Figure S2a. ${ }^{1} \mathrm{H}-\mathrm{NMR}$ spectra of $\mathbf{4}$ (top) and $\mathbf{2}$ (bottom) in $\mathrm{CDCl}_{3}(400 \mathrm{MHz})$.

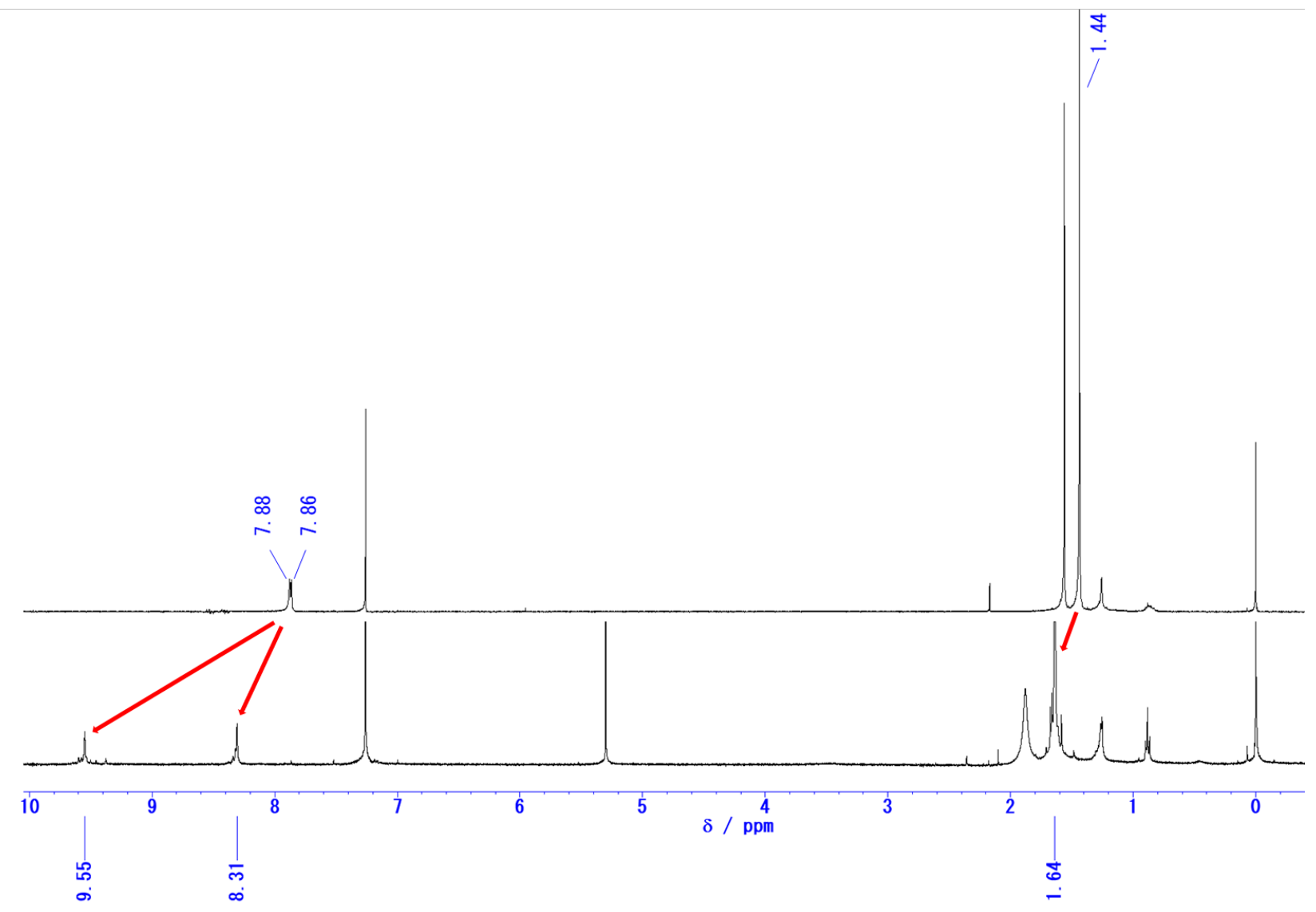

Figure S2b. ${ }^{1} \mathrm{H}-\mathrm{NMR}$ spectra of $\mathbf{2}$ (top) and $\mathbf{2}^{2+}$ (bottom) in $\mathrm{CDCl}_{3}(400 \mathrm{MHz})$. 


\section{X-ray crystal structures of $4,2,2^{++}$, and $2^{2+}$}

X-ray diffraction data were taken on Rigaku Varimax with RAPID diffractometer using multilayer mirror monochromated $\mathrm{Cu}-\mathrm{K} \alpha$ irradiation $(\lambda=1.54187 \AA)$ or Rigaku Varimax with Satturn diffractometer using multilayer mirror monochromated Mo K $\alpha$ radiation $(\lambda=0.71073 \AA)$ at $100 \pm 1 \mathrm{~K}$. The crystals were mounted in cryoloops. Data collection was performed using CrystalClear software. Data reduction was performed using RAPID AUTO or CrystalClear or CrysAlisPro software. The data were corrected for Lorentz polarization and absorption effects. The structures were solved using SHELXT 2014/5 $5^{[\mathrm{S} 1]}$ or SIR2004 ${ }^{[\mathrm{S} 2]}$ or SHELXD97 ${ }^{[\mathrm{S} 3]}$ and expanded using the Fourier technique. All calculations were performed using Rigaku Crystal-Structure crystallographic software package. SHELXL-2016/6 ${ }^{[\mathrm{S} 4]}$ or SHELXL-2017/1 was used for structure refinement. The data were validated using PLATON. Disorderd solvents in the crystal structures of $\mathbf{4 , 2}$ and $\mathbf{2}^{\mathbf{2 +}}$ were removed by SQUEEZE. H atoms of all X-ray structures were omitted for clarity.
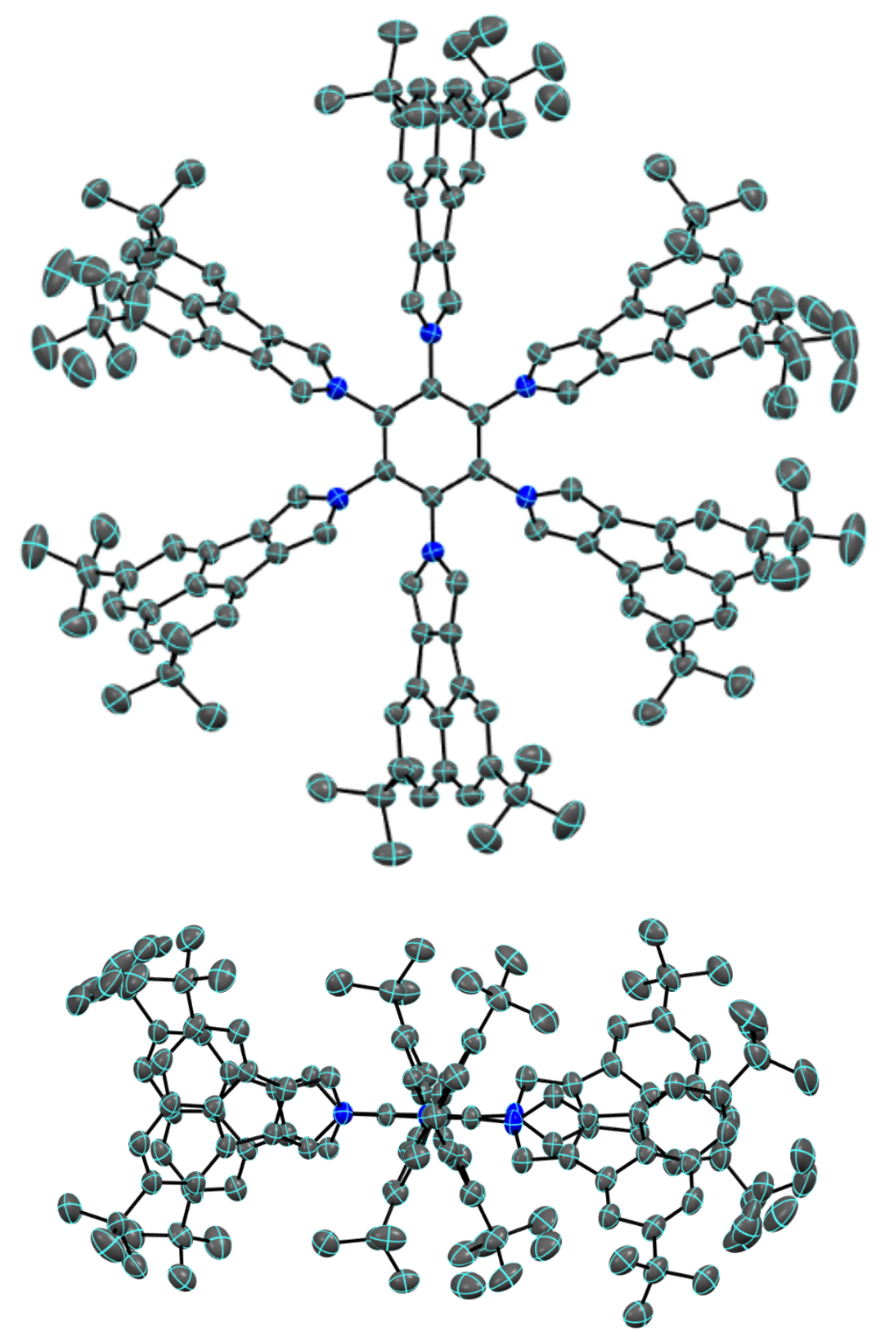

Figure S3a. Crystal structure of $\mathbf{4}$ with thermal ellipsoids drawn at 50\% probability level (top: top view, bottom: side view). 
Table S1. Crystallographic refinement data for 4 .

\section{Formula}

Formula Weight

Size

Temperature

Crystal System

Space Group

Unit cell dimensions

Volume

Z

Density (calculated)

$\mathrm{R}_{\text {int }}$

$\mathrm{R}_{1}$ [I $\left.>2 \operatorname{sigma}(\mathrm{I})\right]$

$\mathrm{wR}_{2}$ (All reflections)

GOF

CCDC No.
$\mathrm{C}_{138} \mathrm{H}_{144} \mathrm{~N}_{6}$

1886.70

$0.250 \times 0.160 \times 0.100 \mathrm{~mm}^{3}$

$100 \mathrm{~K}$

monoclinic

C2/c (\#15)

$\mathrm{a}=35.7965(10) \AA, \mathrm{b}=20.9573(6) \AA, \mathrm{c}=20.7203(6) \AA$

$\alpha=90.0000^{\circ}, \beta=109.467(8)^{\circ}, \gamma=90.0000^{\circ}$

14655.7(10) $\AA^{3}$

4

$0.855 \mathrm{~g} / \mathrm{cm}^{3}$

0.0624

0.0652

0.2055

1.080

1978361 


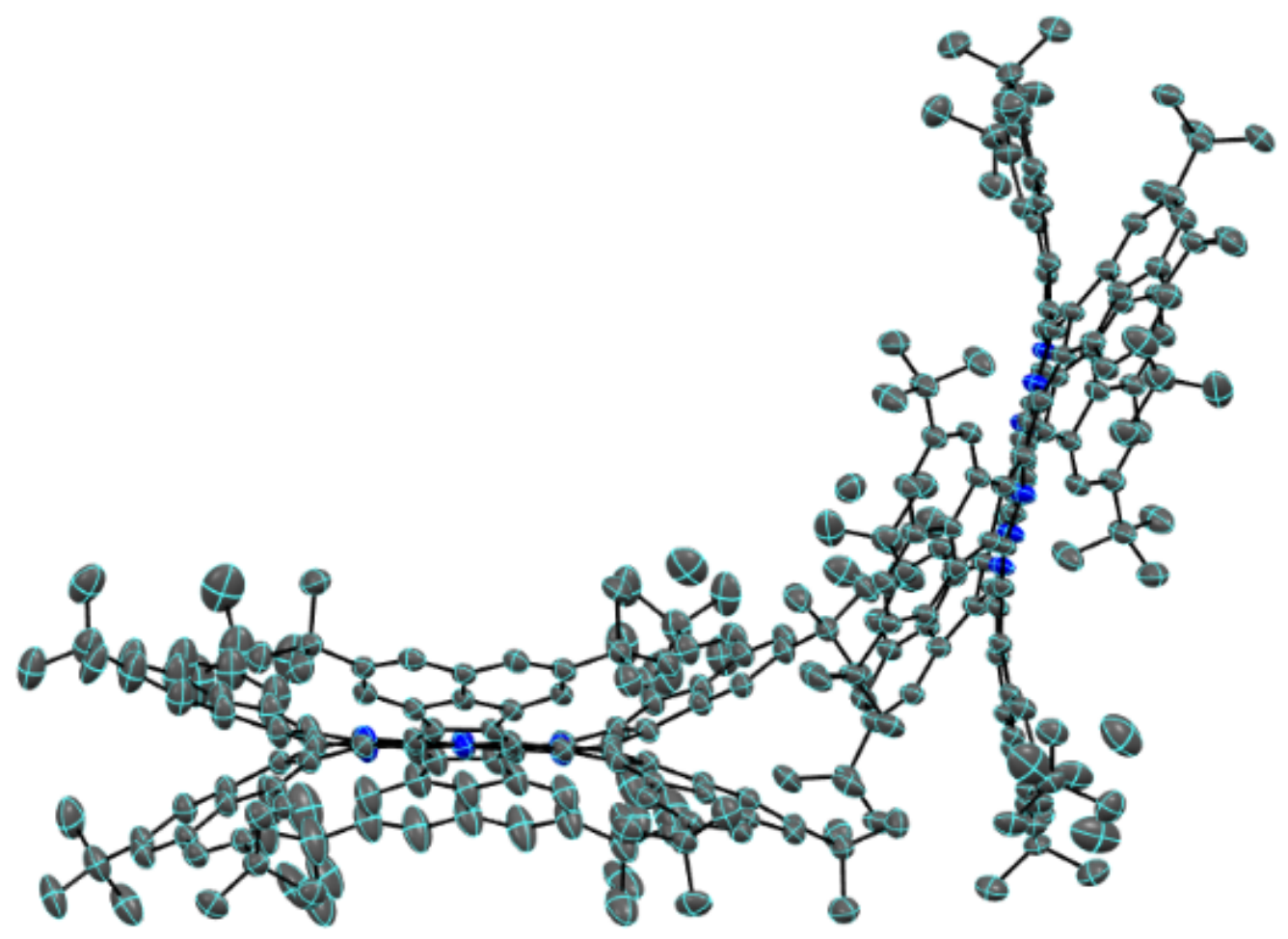

Figure S3b. Crystal structure of $\mathbf{2}$ with thermal ellipsoids drawn at 50\% probability level.

Table S2. Crystallographic refinement data for $\mathbf{2}$.

$\begin{array}{ll}\text { Formula } & \mathrm{C}_{138} \mathrm{H}_{132} \mathrm{~N}_{6} \\ \text { Formula weight } & 1874.60 \\ \text { Size } & 0.280 \times 0.140 \times 0.030 \mathrm{~mm}^{3} \\ \text { Temperature } & 100 \mathrm{~K} \\ \text { Crystal system } & \text { monoclinic } \\ \text { Space group } & \mathrm{C} 2 / \mathrm{c}(\# 15) \\ \text { Unit cell dimensions } & \mathrm{a}=35.9195(7) \AA, \mathrm{b}=47.3361(9) \AA, \mathrm{c}=27.0025(5) \AA \\ & \alpha=90.0000, \beta=131.609(9), \gamma=90.0000 \\ \text { Volume } & 34328(5) \AA^{3} \\ Z & 8 \\ \text { Density (calculated) } & 0.725 \mathrm{~g} \cdot \mathrm{cm}^{-3} \\ \text { Rint }_{\text {R }} & 0.0540 \\ \mathrm{R}_{1}[\mathrm{I}>2 \text { sigma(I)] } & 0.0524 \\ \text { wR } 2 \text { (All reflections) } & 0.1592 \\ \text { GOF } & 1.002 \\ \text { CCDC No. } & 1911124\end{array}$




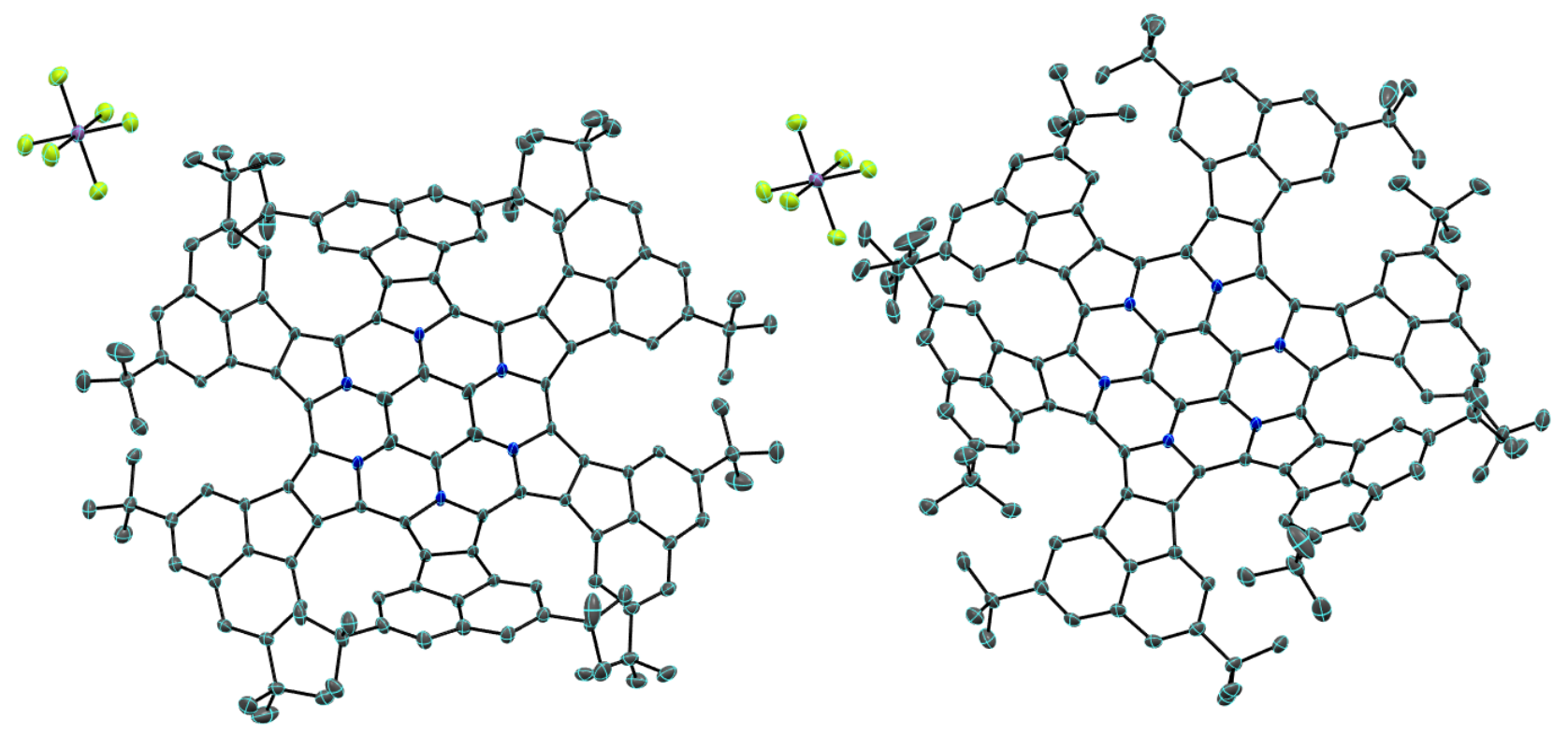

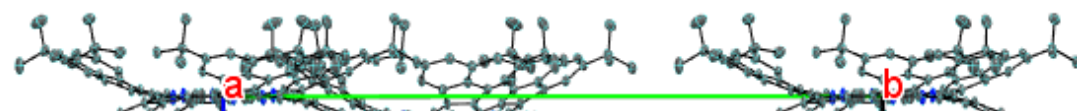
Y

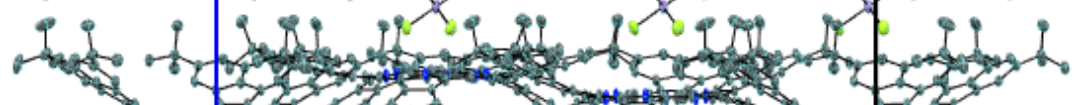
Y

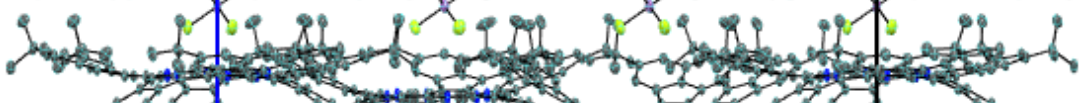

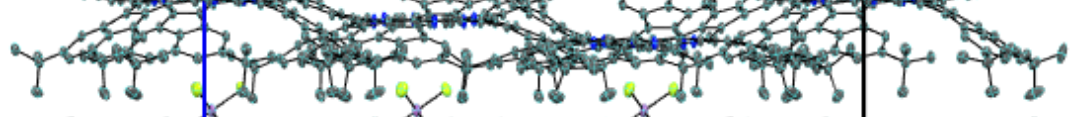

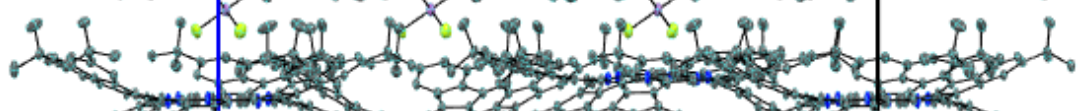
Y

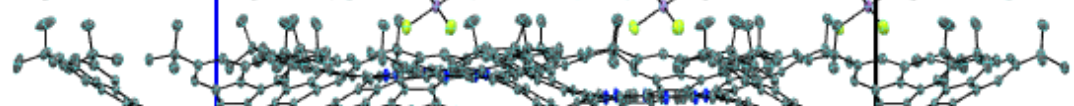
Y ins Y

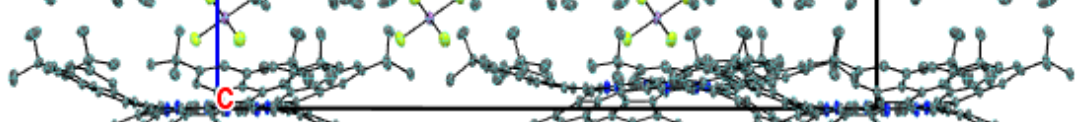
Y 


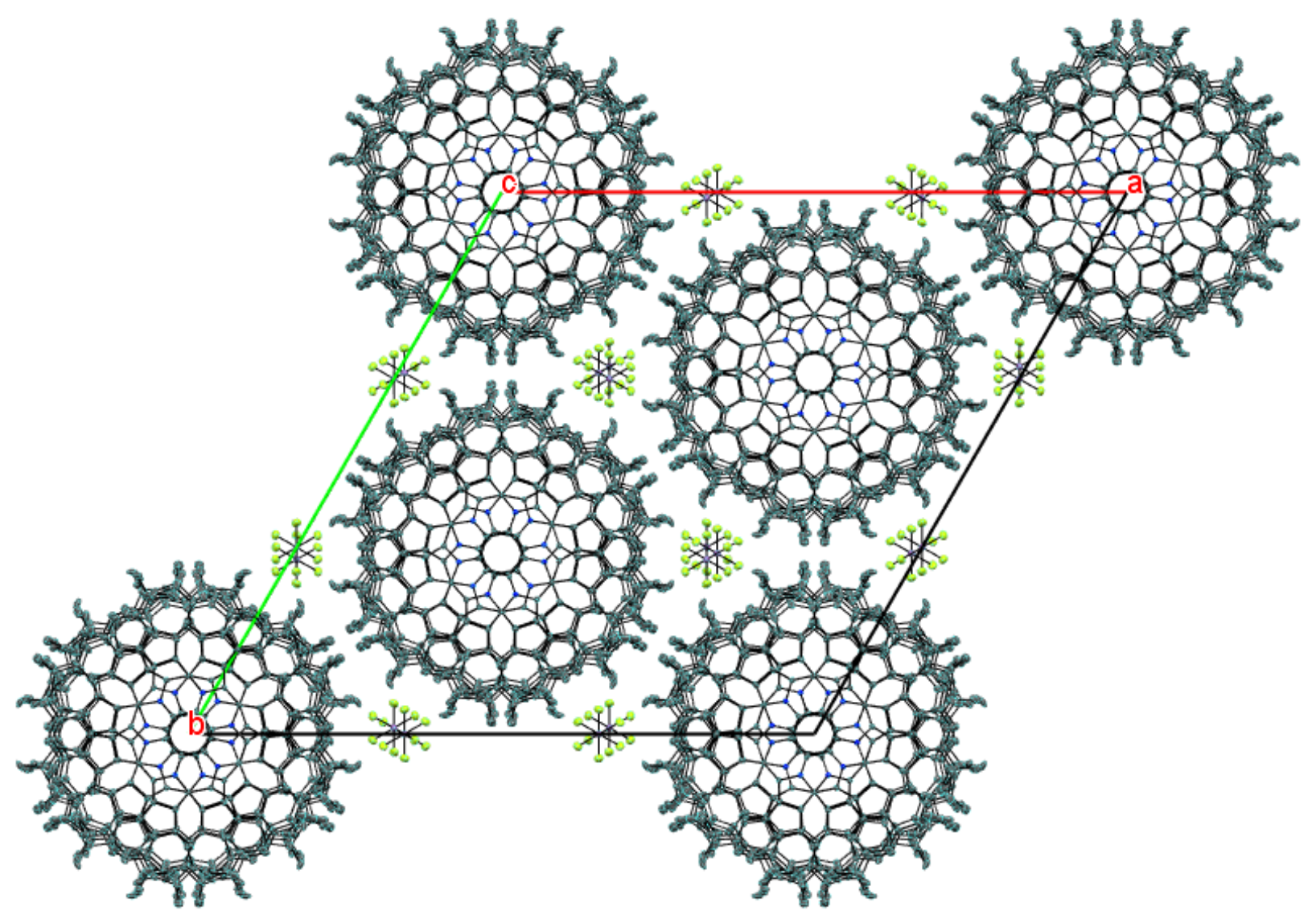

Figure S3c. Crystal structure of $\mathbf{2}^{\circ+}$ with thermal ellipsoids drawn at 50\% probability level (Top: crystallographically independent two molecules, middle: $a$ axis in the packing structure, bottom: $c$ axis in the packing structure). Solvent molecules were omitted for clarify.

Table S3. Crystallographic refinement data for $\mathbf{2}^{\cdot+}$.

$\begin{array}{ll}\text { Formula } & \mathrm{C}_{138} \mathrm{H}_{132} \mathrm{~N}_{6}, 10\left(\mathrm{C}_{6} \mathrm{H}_{5} \mathrm{Cl}\right), \mathrm{F}_{6} \mathrm{Sb} \\ \text { Formula weight } & 3235.93 \\ \text { Size } & 0.150 \times 0.140 \times 0.040 \mathrm{~mm}^{3} \\ \text { Temperature } & 100 \mathrm{~K} \\ \text { Crystal system } & \text { trigonal } \\ \text { Space group } & R \overline{3} \mathrm{c}(\# 167) \\ \text { Unit cell dimensions } & \mathrm{a}=39.3597(2) \AA, \mathrm{b}=39.3597(2) \AA, \mathrm{c}=55.9666(6) \AA \\ & \alpha=90.0000, \beta=90.0000, \gamma=120.0000 \\ \text { Volume } & 75086.7(10) \AA^{3} \\ Z & 18 \\ \text { Density (calculated) } & 1.288 \mathrm{~g} \cdot \mathrm{cm}^{-3} \\ \mathrm{R}_{\text {int }} & 0.0797 \\ \mathrm{R}_{I}[\mathrm{I}>2 \text { sigma(I)] } & 0.0915 \\ \text { wR } & \text { (All reflections) } \\ \text { GOF } & 0.2420 \\ \text { CCDC No. } & 1.332 \\ & 1911125\end{array}$



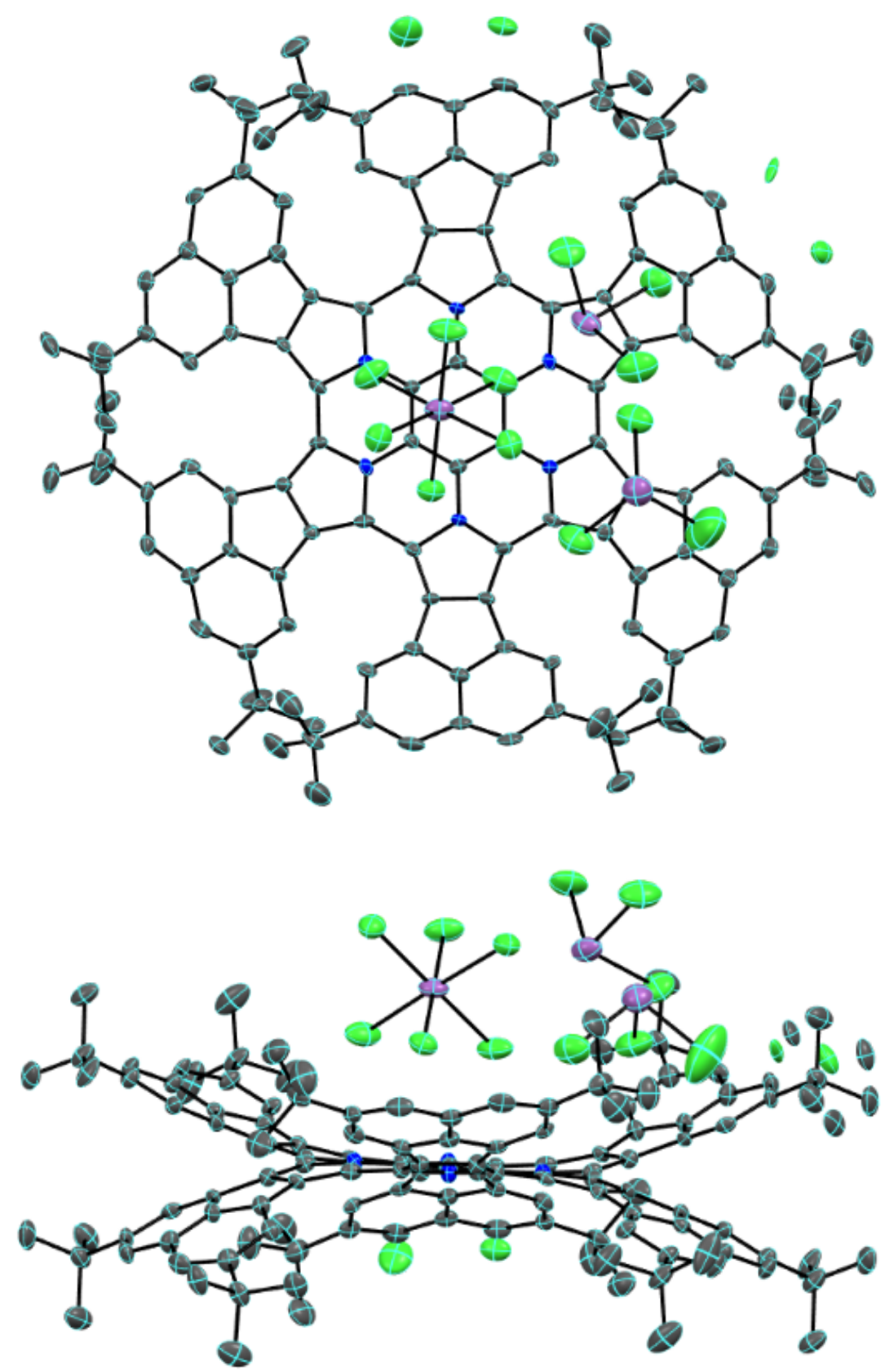

Figure S3d. Crystal structure of $\mathbf{2}^{2+}$ with thermal ellipsoids drawn at 50\% probability level (top: top view, bottom: side view). 
Table S4. Crystallographic refinement data for $\mathbf{2}^{2+}$.

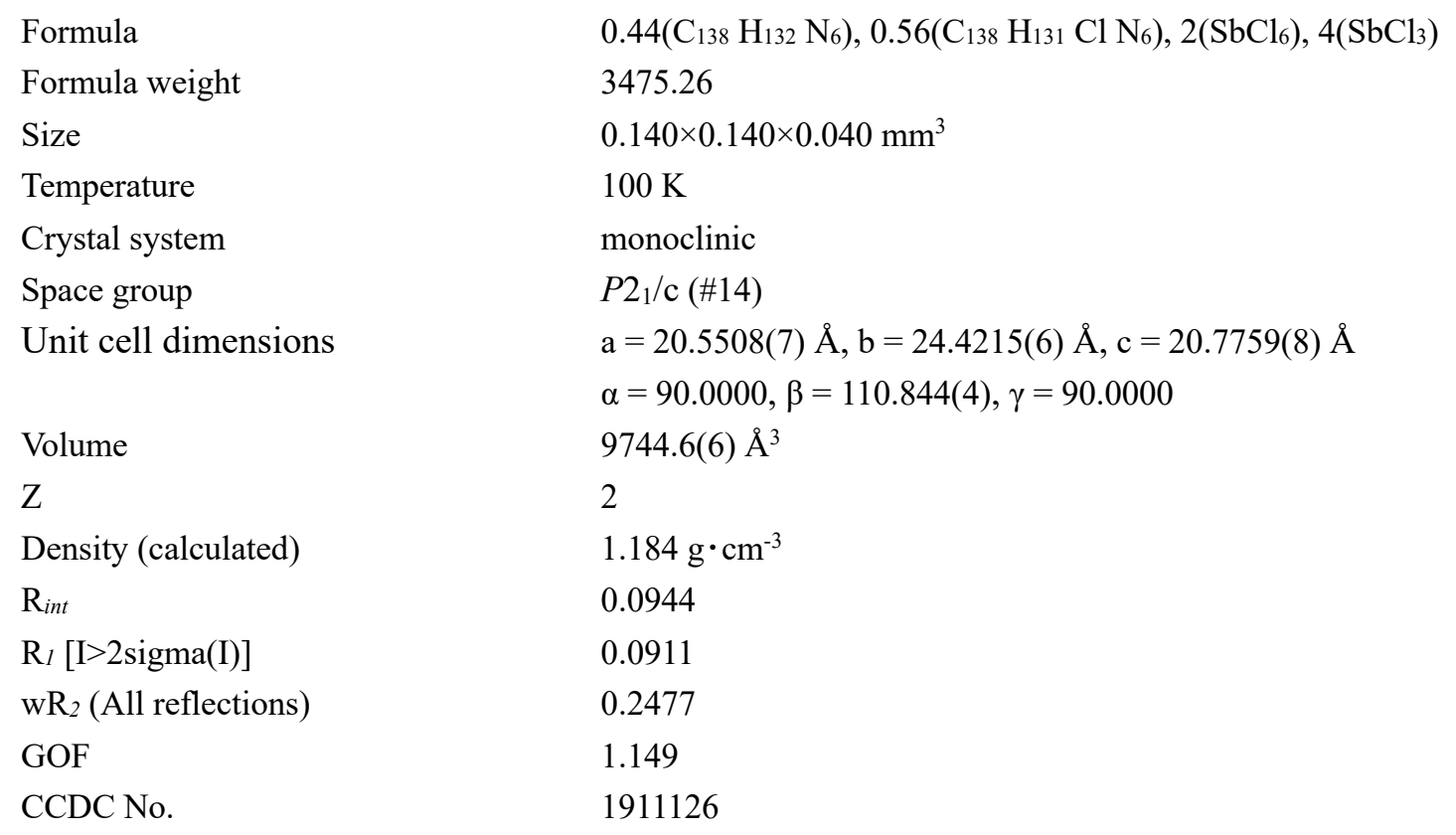


4. Comparison of bowl depths of $2,2^{\cdot+}$, and $2^{2+}$
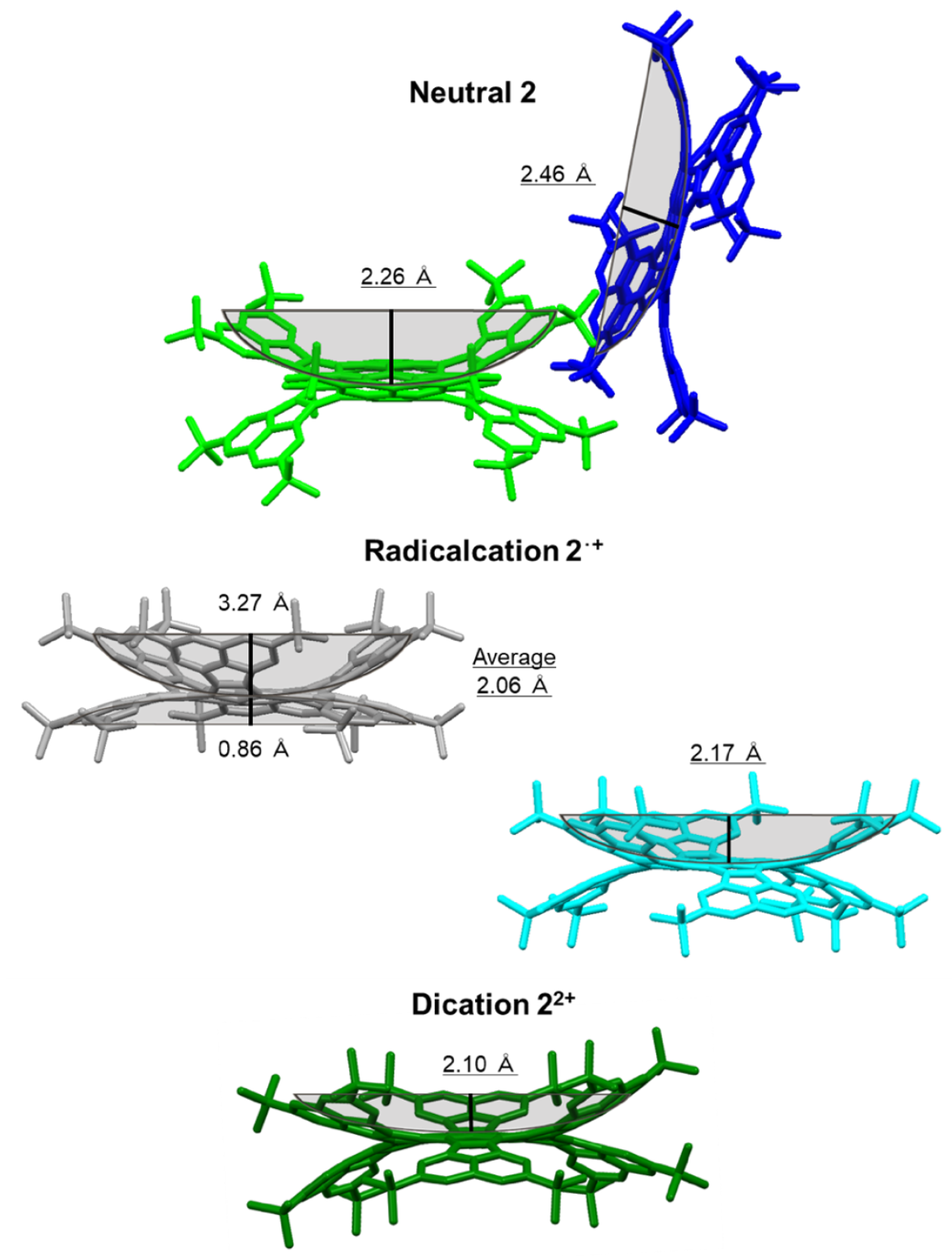

Figure S4. Bowl depths of $\mathbf{2 ,} \mathbf{2}^{\mathbf{}}$, and $\mathbf{2}^{2+}$.

Table S5. Bowl depths of $\mathbf{2}$ in the X-ray and DFT-optimized structures.

\begin{tabular}{|c|c|c|}
\hline & $\begin{array}{c}2 \\
(\mathrm{X}-\mathrm{ray}, \AA)\end{array}$ & $\begin{array}{c}\mathbf{2 - H} \\
(\mathrm{DFT} \text { calculation, } \AA \text { ) }\end{array}$ \\
\hline Neutral & $2.26,2.46$ & 1.902 \\
\hline $\begin{array}{c}\text { Radical } \\
\text { cation }\end{array}$ & $2.06,2.17$ & 1.901 \\
\hline Dication & 2.10 & 1.906 \\
\hline
\end{tabular}




\section{Intermolecular interaction of 2 in the packing structure}

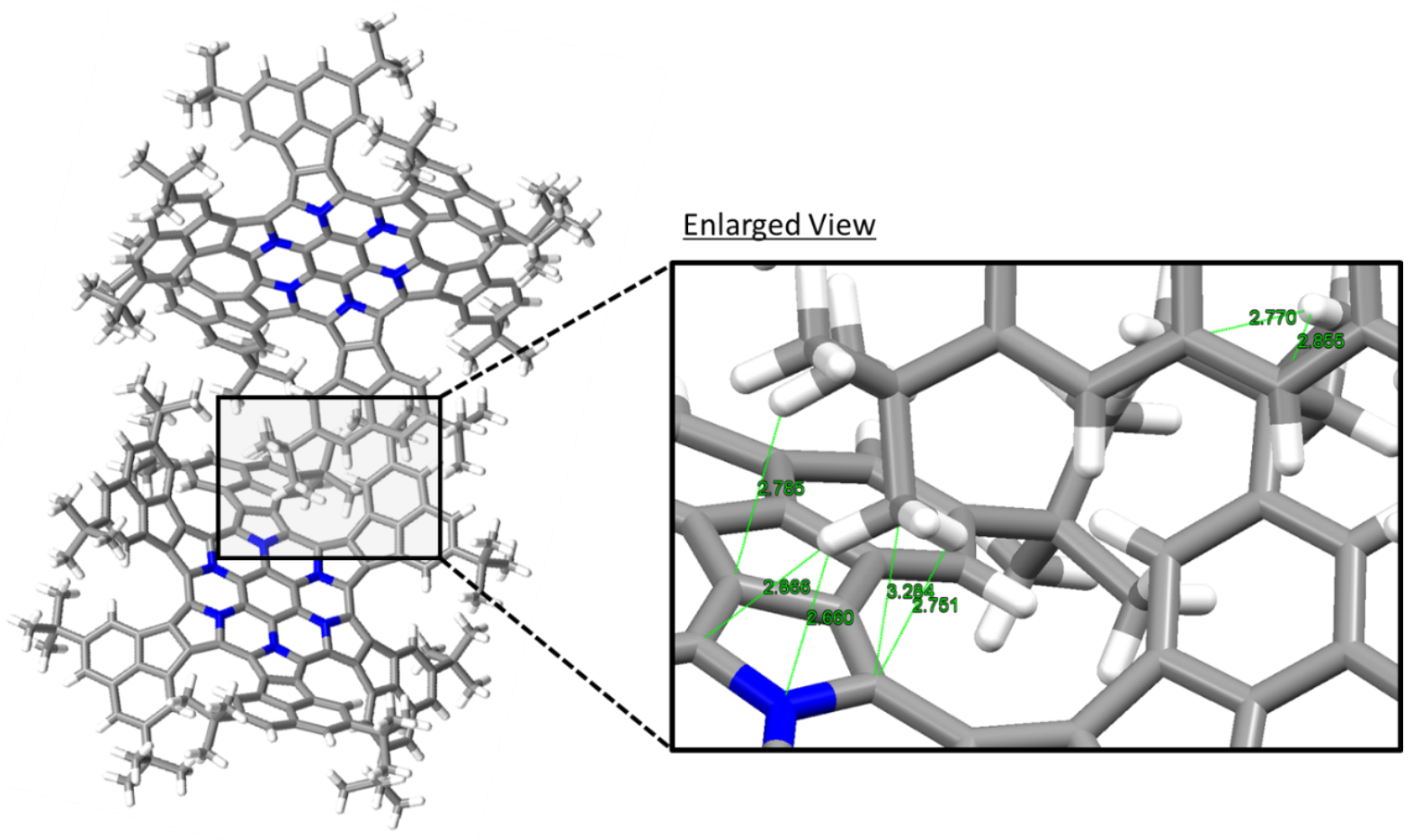

Figure S5. Intermolecular interaction of $\mathbf{2}$ in the packing structure. 


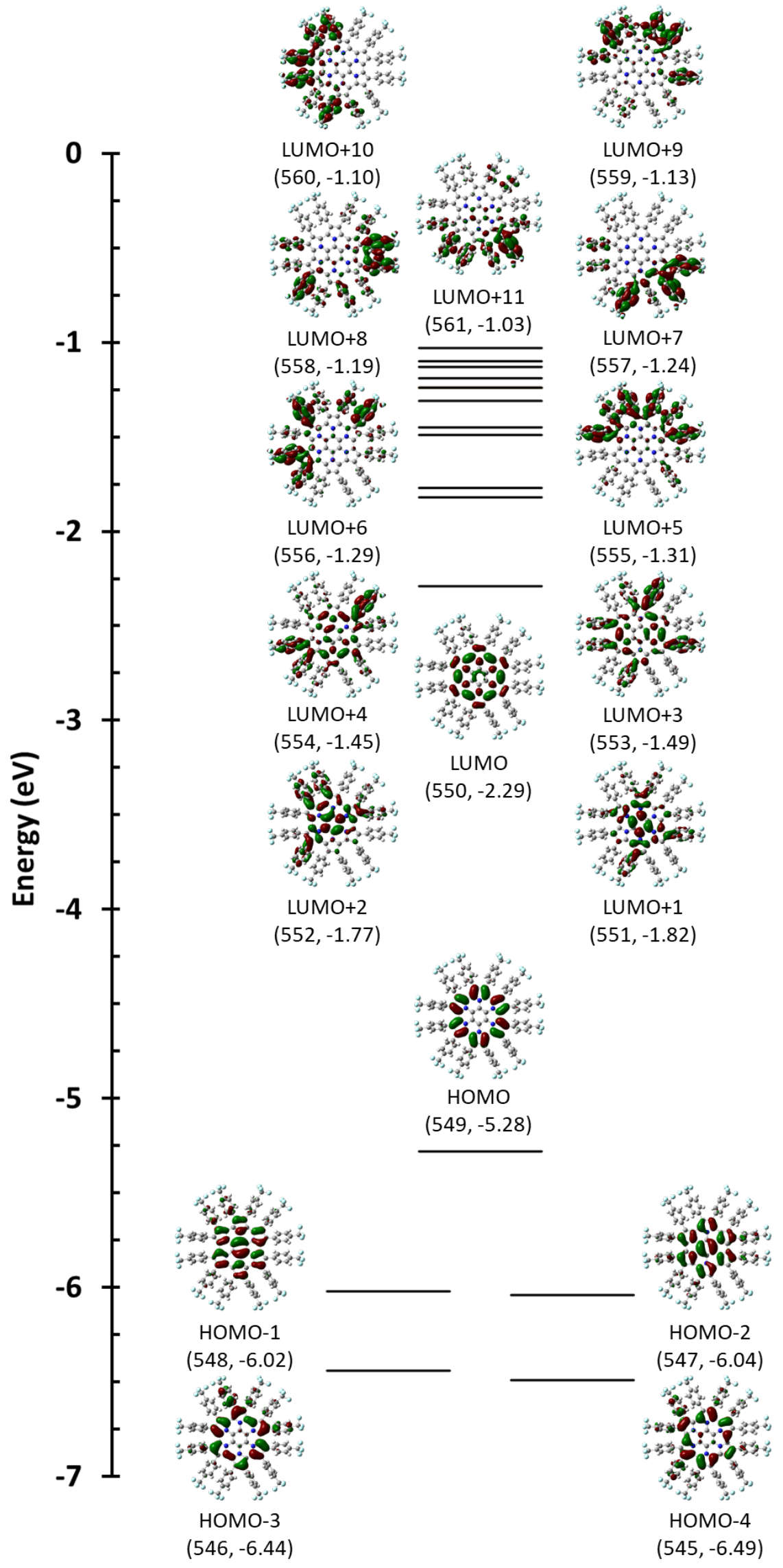

Figure S6a. Molecular orbital diagram of 1a (Symmetry: $C_{1}$ (crystal structure), Basis set: B3LYP/6-31G(d)). 


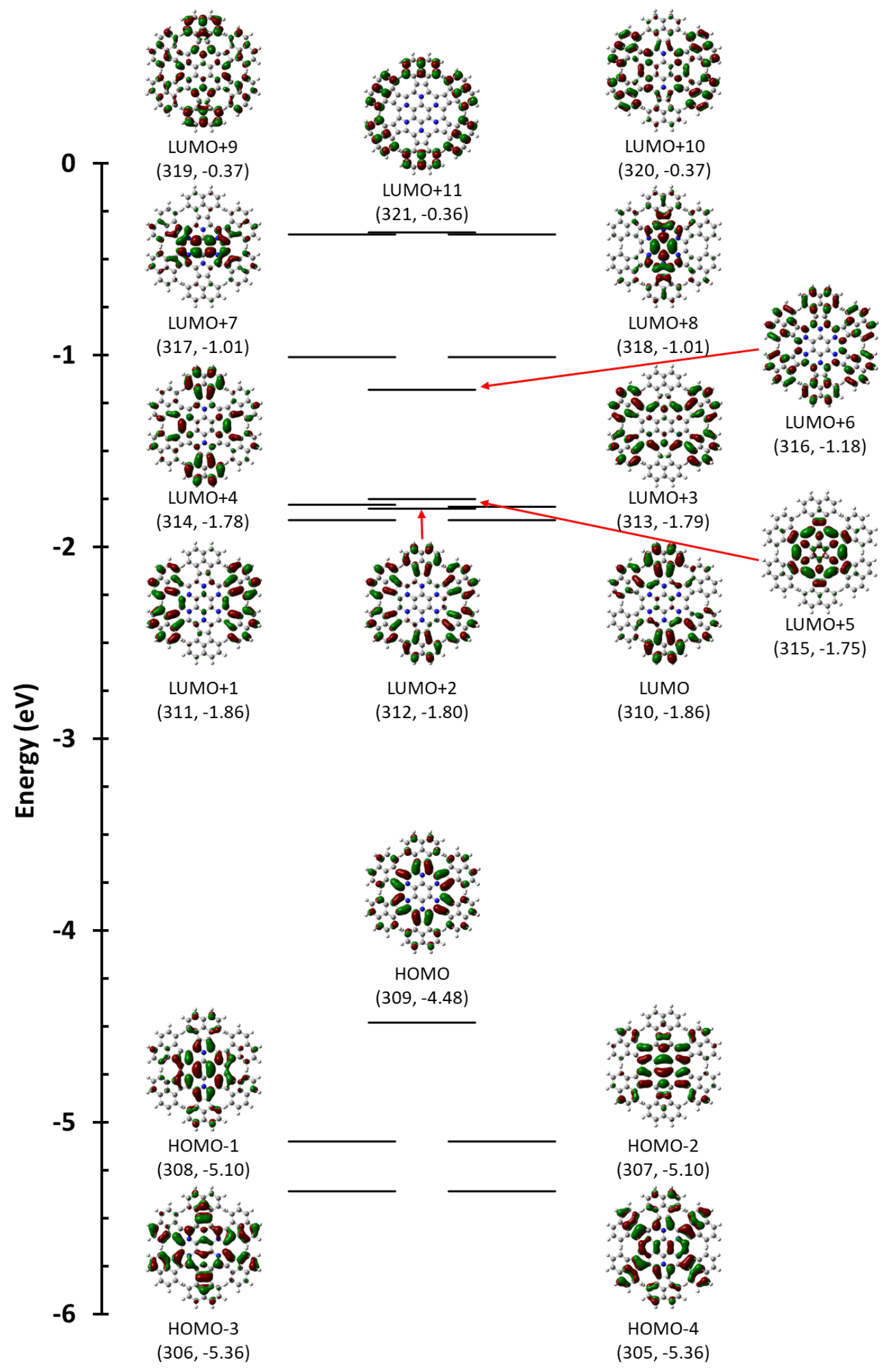

Figure S6b. Molecular orbital diagram of 2-H (Symmetry: $C_{\mathrm{i}}$, Basis set: B3LYP/6-31G(d)). 


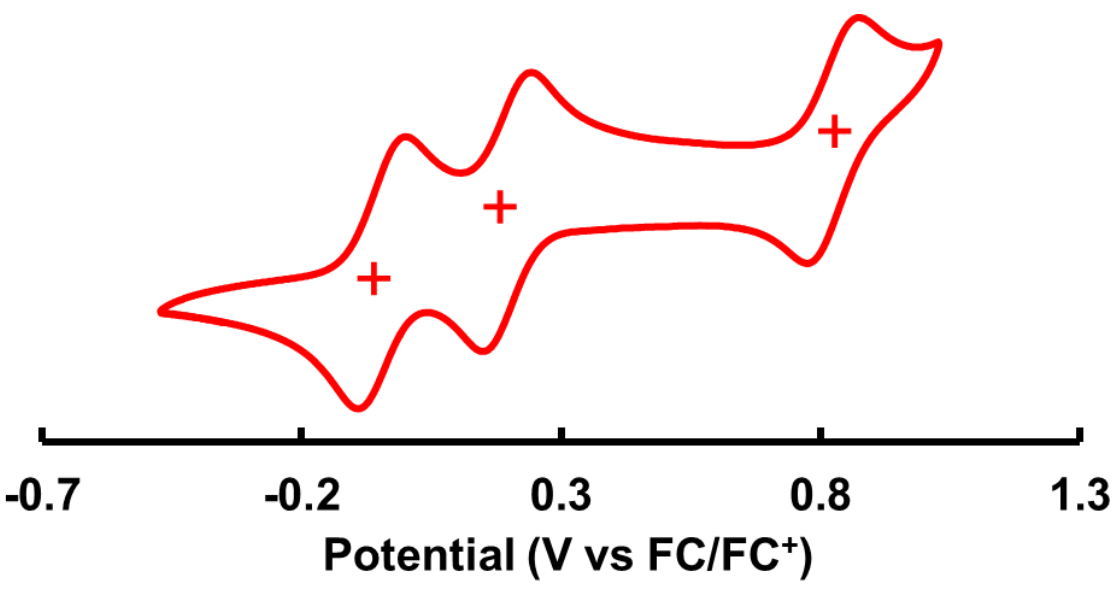

Figure S7. Cyclic voltammogram of 2 in $\mathrm{CH}_{2} \mathrm{Cl}_{2}$. Conditions: solvent, $\mathrm{CH}_{2} \mathrm{Cl}_{2}$, supporting electrolyte, $0.1 \mathrm{M}$ $\mathrm{TBAPF}_{6}$; working electrode, $\mathrm{Pt}$; reference electrode, $\mathrm{Ag} / \mathrm{AgNO}_{3}$; counter electrode, Pt wire; scan rate, $100 \mathrm{mV} / \mathrm{s}$, potentials, vs $\mathrm{Fc} / \mathrm{Fc}^{+}$.

Table S6. Potential data of 2.

\begin{tabular}{|c|c|c|c|c|}
\hline & $E^{0 \times 1}{ }_{1 / 2}(\mathrm{~V})$ & $E^{0 \times 2}{ }_{1 / 2}(\mathrm{~V})$ & $E^{0 \times 3}{ }_{1 / 2}(\mathrm{~V})$ & $E^{0 \times 3}{ }_{1 / 2}-E^{0 \times 3}{ }_{1 / 2}(\mathrm{~V})$ \\
\hline 2 & -0.08 & 0.16 & 0.79 & 0.63 \\
\hline
\end{tabular}




\section{Stepwise oxidation of 2 with $\mathrm{SbCl}_{5}$}

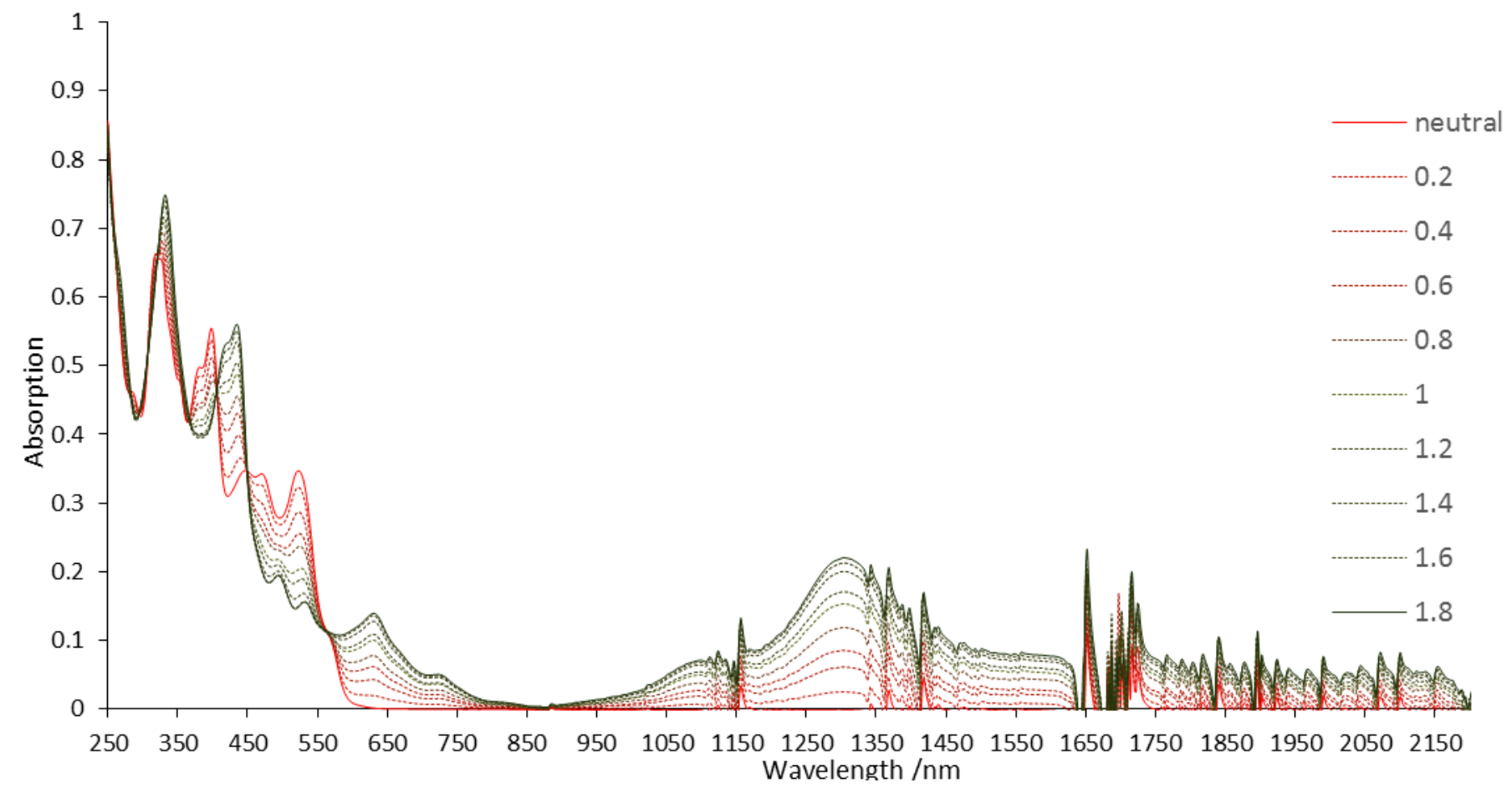

Figure S8a. UV-Vis-NIR spectra of 2 in $\mathrm{CH}_{2} \mathrm{Cl}_{2}\left(5.44 \times 10^{-6} \mathrm{M}\right)$ from neutral to radical cation states.

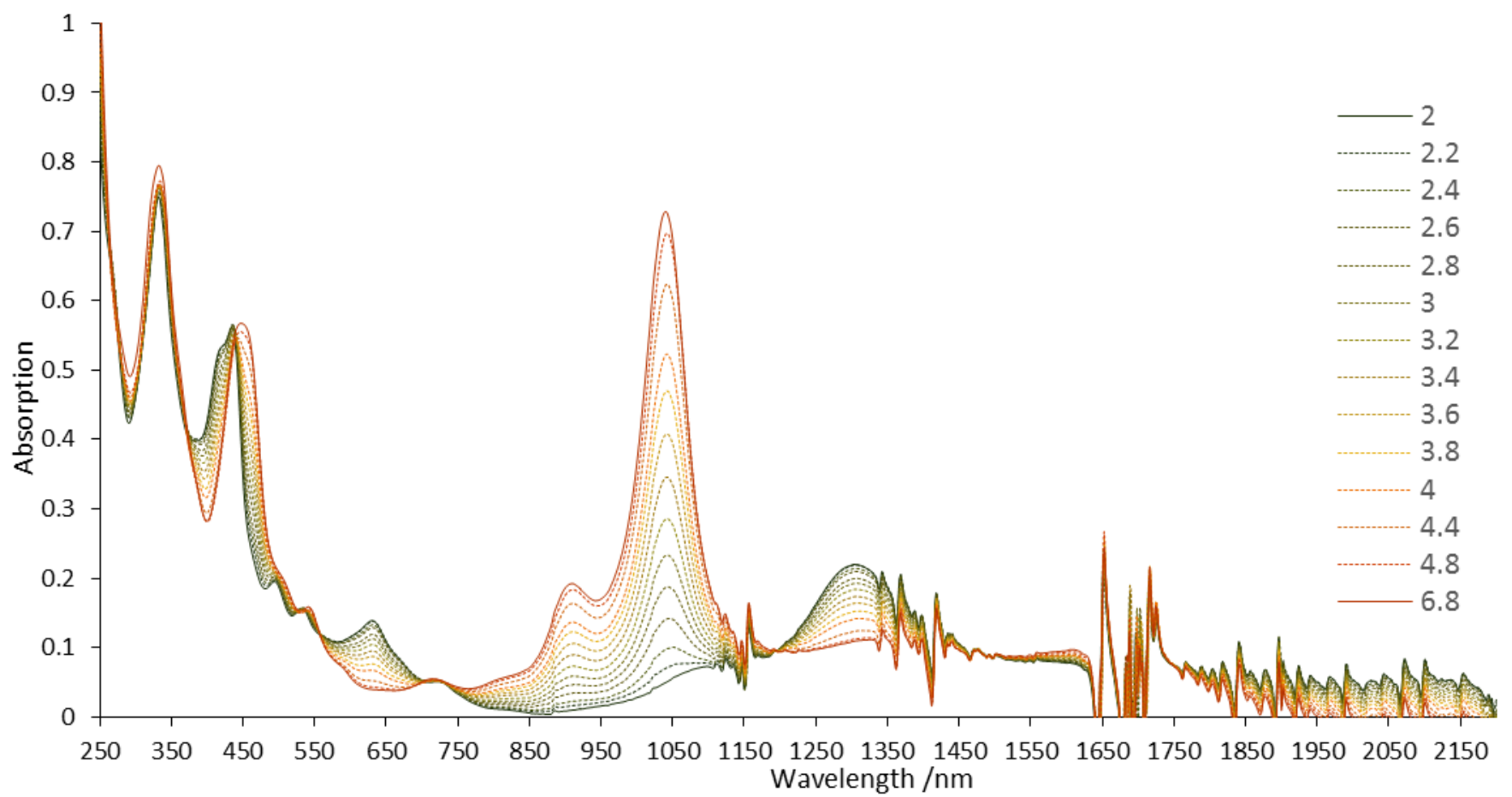

Figure S8b. UV-Vis-NIR spectra of 2 in $\mathrm{CH}_{2} \mathrm{Cl}_{2}\left(5.44 \times 10^{-6} \mathrm{M}\right)$ from radical cation to dication states. 
9. ESR spectra of $2^{\cdot+}$ and $2^{2+}$, and spin density map of $2-\mathrm{H}^{\cdot+}$

- Radical cation (203 K)

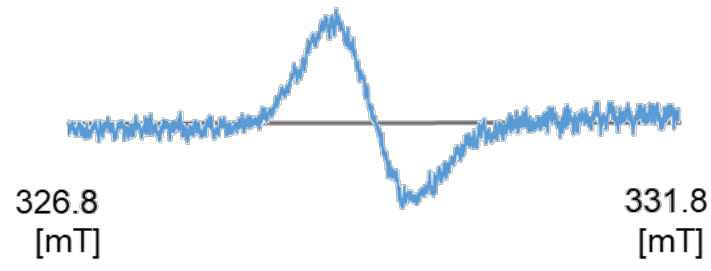

- Dication (273 K)

326.8

[mT]

331.8

$[\mathrm{mT}]$

$g=2.0020, \Delta H=0.45 \mathrm{mT}$

Figure S9a. ESR spectra of $\mathbf{2}^{\cdot+}$ (left) and $\mathbf{2}^{2+}$ (right) in $\mathrm{CH}_{2} \mathrm{Cl}_{2}$.

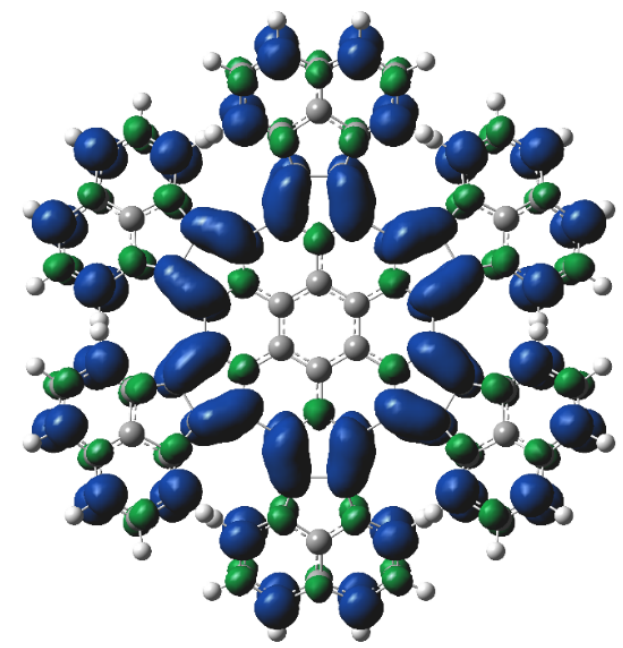

Figure S9b. Spin density of $\mathbf{2 - \mathbf { H } ^ { + + }}$ (Isovalue $=0.0004$, Basis set: UBL3YP/6-31G(d)). 


\section{NICS values of $1 \mathrm{~b}, 2-\mathrm{H}$, and $2-\mathrm{H}^{2+}$}

The calculations were performed at $\mathrm{HF} / 6-311+\mathrm{G}(\mathrm{d}, \mathrm{p}) / / \mathrm{B} 3 \mathrm{LYP} / 6-31 \mathrm{G}(\mathrm{d})$.
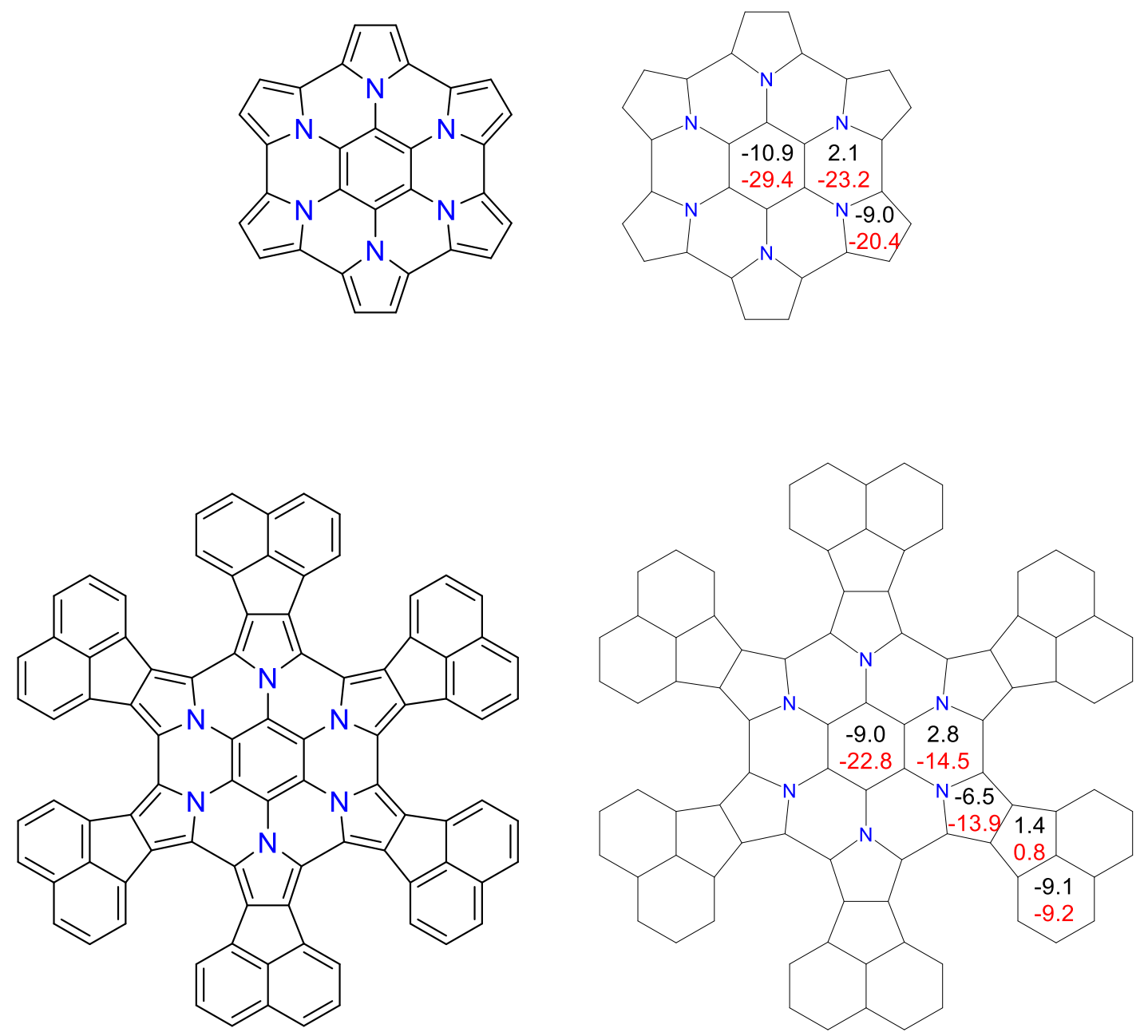

Figure S10. NICS(1) iso of HPHAC 1b and 2-H (Black: Neutral, Red: Dication). 
Table S7. NICS values

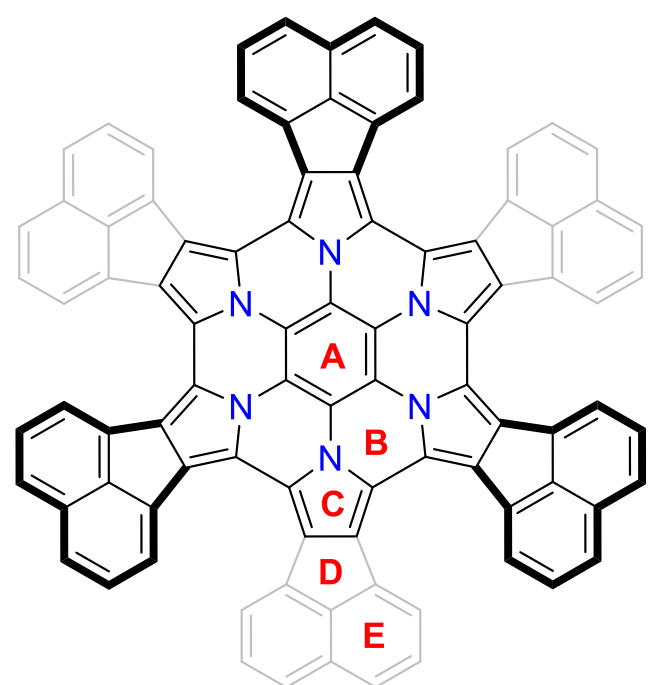

\begin{tabular}{|c|c|c|c|c|c|}
\hline Neutral 2-H & A & B & C & D & E \\
\hline NICS $(+1)_{\text {iso }}$ & -9.00 & 2.77 & -6.52 & 1.37 & -9.10 \\
\hline NICS $(0)_{\text {iso }}$ & -11.85 & 4.44 & -9.55 & 3.87 & -8.25 \\
\hline NICS(-1) iso & -9.00 & 2.77 & -6.56 & 0.12 & -10.57 \\
\hline
\end{tabular}

\begin{tabular}{|c|c|c|c|c|c|}
\hline Dication 2-H & A & B & C & D & E \\
\hline NICS $(+1)_{\text {iso }}$ & -22.75 & -14.53 & -9.37 & 2.99 & -7.17 \\
\hline NICS $(0)_{\text {iso }}$ & -27.05 & -16.03 & -13.44 & 4.22 & -6.22 \\
\hline NICS $(-1)_{\text {iso }}$ & -22.75 & -14.53 & -13.89 & 0.80 & -9.18 \\
\hline
\end{tabular}




\section{TD-DFT calculations of $2-\mathrm{H}, 2-\mathrm{H}^{++}$, and $2-\mathrm{H}^{2+}$}

The calculations were performed at the R(U)B3LYP/6-31G(d)//R(U)B3LYP/6-31G(d) level of theory.

Neutral 2-H

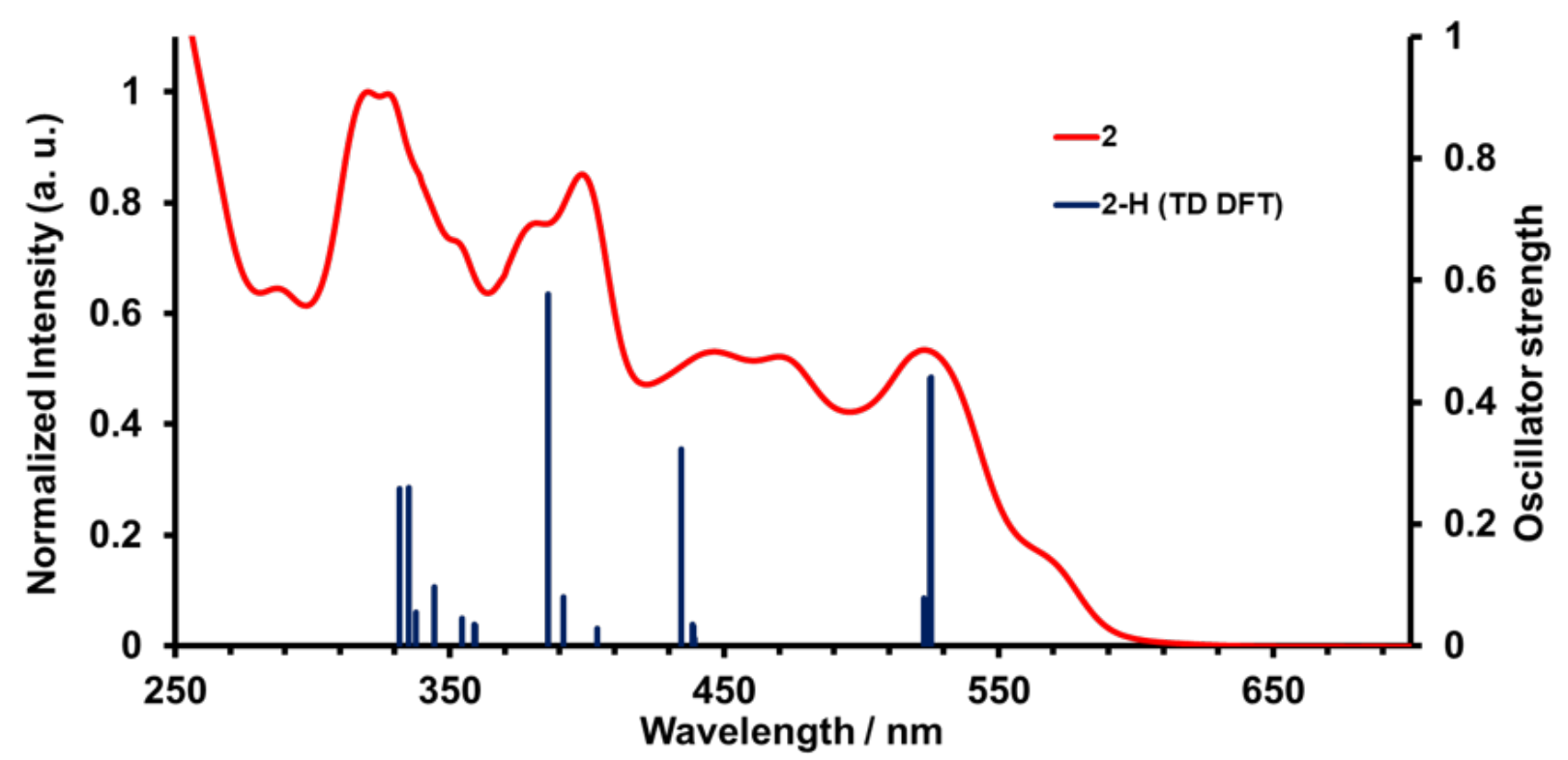

Figure S11a. UV-Vis-NIR spectra of $\mathbf{2}$ (red line) in $\mathrm{CH}_{2} \mathrm{Cl}_{2}$ and TD-DFT calculation of $\mathbf{2 - H}$ (blue line).

Table S8. TD-DFT calculation result of 2-H.

$\mathrm{HOMO}=309, \mathrm{LUMO}=310$

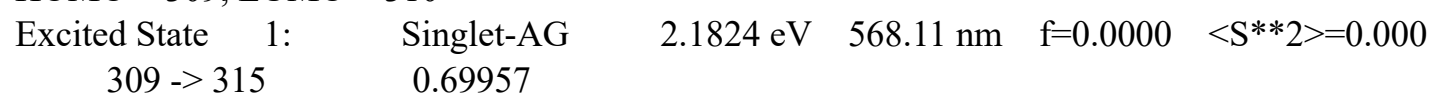

This state for optimization and/or second-order correction.

Total Energy, E(TD-HF/TD-KS) = -3779.80214981

Copying the excited state density for this state as the 1-particle RhoCI density.

\begin{tabular}{|c|c|c|c|c|c|c|}
\hline $\begin{array}{l}\text { Excited State } \\
\qquad 309->310\end{array}$ & $2:$ & $\begin{array}{l}\text { Singlet-AG } \\
0.70199\end{array}$ & $2.3137 \mathrm{eV}$ & $535.88 \mathrm{~nm}$ & $\mathrm{f}=0.0000$ & $<\mathrm{S}^{* * 2}>=0.000$ \\
\hline $\begin{array}{l}\text { Excited State } \\
\qquad 309->311\end{array}$ & 3: & $\begin{array}{l}\text { Singlet-AG } \\
0.70198\end{array}$ & $2.3143 \mathrm{eV}$ & $535.74 \mathrm{~nm}$ & $\mathrm{f}=0.0000$ & $<\mathrm{S} * * 2>=0.000$ \\
\hline $\begin{array}{l}\text { Excited State } \\
\quad 308->315\end{array}$ & 4: & $\begin{array}{l}\text { Singlet-AU } \\
-0.11806\end{array}$ & $2.3605 \mathrm{eV}$ & $525.26 \mathrm{~nm}$ & $\mathrm{f}=0.4402$ & $<\mathrm{S} * * 2>=0.000$ \\
\hline $309->313$ & & 0.68561 & & & & \\
\hline $\begin{array}{l}\text { Excited State } \\
\qquad 307->315\end{array}$ & 5: & $\begin{array}{l}\text { Singlet-AU } \\
0.11792\end{array}$ & $2.3613 \mathrm{eV}$ & $525.07 \mathrm{~nm}$ & $\mathrm{f}=0.4391$ & $<\mathrm{S} * * 2>=0.000$ \\
\hline $309->314$ & & 0.68562 & & & & \\
\hline $\begin{array}{l}\text { Excited State } \\
\qquad 309->312\end{array}$ & 6: & $\begin{array}{l}\text { Singlet-AU } \\
0.70070\end{array}$ & $2.3713 \mathrm{eV}$ & $522.85 \mathrm{~nm}$ & $f=0.0782$ & $<\mathrm{S}^{* * 2}>=0.000$ \\
\hline $\begin{array}{l}\text { Excited State } \\
\quad 305->311\end{array}$ & 7: & $\begin{array}{l}\text { Singlet-AU } \\
-0.10239\end{array}$ & $2.7717 \mathrm{eV}$ & $447.32 \mathrm{~nm}$ & $\mathrm{f}=0.0000$ & $<\mathrm{S} * * 2>=0.000$ \\
\hline $306->310$ & & 0.10278 & & & & \\
\hline $307->310$ & & 0.47572 & & & & \\
\hline $308->311$ & & 0.47679 & & & & \\
\hline $\begin{array}{l}\text { Excited State } \\
\qquad 307->311\end{array}$ & 8: & $\begin{array}{l}\text { Singlet-AU } \\
-0.47453\end{array}$ & $2.8225 \mathrm{eV}$ & $439.27 \mathrm{~nm}$ & $f=0.0127$ & $<\mathrm{S}^{* * 2}>=0.000$ \\
\hline
\end{tabular}




\begin{tabular}{|c|c|c|c|c|c|}
\hline $308->310$ & 0.49870 & & & & \\
\hline Excited State 9: & Singlet-AU & $2.8264 \mathrm{eV}$ & $438.66 \mathrm{~nm}$ & $\mathrm{f}=0.0327$ & $<\mathrm{S} * * 2>=0.000$ \\
\hline $308->315$ & 0.67111 & & & & \\
\hline $309->313$ & 0.12929 & & & & \\
\hline Excited State $10:$ & Singlet-AU & $2.8273 \mathrm{eV}$ & $438.53 \mathrm{~nm}$ & $\mathrm{f}=0.0346$ & $<\mathrm{S} * * 2>=0.000$ \\
\hline $307->315$ & 0.67156 & & & & \\
\hline $309->314$ & -0.12985 & & & & \\
\hline Excited State 11: & Singlet-AG & $2.8322 \mathrm{eV}$ & $437.77 \mathrm{~nm}$ & $\mathrm{f}=0.0000$ & $<\mathrm{S} * * 2>=0.000$ \\
\hline $304->310$ & 0.10527 & & & & \\
\hline $307->312$ & 0.29807 & & & & \\
\hline $307->313$ & -0.16205 & & & & \\
\hline $307->314$ & 0.22203 & & & & \\
\hline $308->312$ & -0.10100 & & & & \\
\hline $308->313$ & 0.49827 & & & & \\
\hline $308->314$ & 0.14322 & & & & \\
\hline $309->318$ & 0.12665 & & & & \\
\hline Excited State $12:$ & Singlet-AG & $2.8323 \mathrm{eV}$ & $437.74 \mathrm{~nm}$ & $\mathrm{f}=0.0000$ & $<\mathrm{S} * * 2>=0.000$ \\
\hline $304->311$ & -0.10979 & & & & \\
\hline $305->312$ & 0.10007 & & & & \\
\hline $307->313$ & -0.37507 & & & & \\
\hline $308->312$ & -0.31036 & & & & \\
\hline $308->313$ & -0.20118 & & & & \\
\hline $308->314$ & 0.38268 & & & & \\
\hline $309->317$ & -0.13359 & & & & \\
\hline Excited State $13:$ & Singlet-AG & $2.8344 \mathrm{eV}$ & $437.42 \mathrm{~nm}$ & $\mathrm{f}=0.0000$ & $<\mathrm{S} * * 2>=0.000$ \\
\hline $307->312$ & 0.10358 & & & & \\
\hline $307->314$ & 0.58462 & & & & \\
\hline $308->313$ & -0.33994 & & & & \\
\hline Excited State $14:$ & Singlet-AU & $2.8540 \mathrm{eV}$ & $434.42 \mathrm{~nm}$ & $\mathrm{f}=0.3206$ & $<\mathrm{S} * * 2>=0.000$ \\
\hline $306->315$ & 0.12365 & & & & \\
\hline $307->310$ & -0.13502 & & & & \\
\hline $307->311$ & 0.46393 & & & & \\
\hline $308->310$ & 0.44530 & & & & \\
\hline $308->311$ & 0.13326 & & & & \\
\hline Excited State $15:$ & Singlet-AU & $2.8541 \mathrm{eV}$ & $434.41 \mathrm{~nm}$ & $\mathrm{f}=0.3225$ & $<\mathrm{S} * * 2>=0.000$ \\
\hline $305->315$ & -0.12277 & & & & \\
\hline $307->310$ & 0.45424 & & & & \\
\hline $307->311$ & 0.13620 & & & & \\
\hline $308->310$ & 0.13142 & & & & \\
\hline $308->311$ & -0.45388 & & & & \\
\hline Excited State $16:$ & Singlet-AG & $2.9147 \mathrm{eV}$ & $425.38 \mathrm{~nm}$ & $\mathrm{f}=0.0000$ & $<\mathrm{S} * * 2>=0.000$ \\
\hline $307->313$ & -0.23253 & & & & \\
\hline $308->312$ & 0.60458 & & & & \\
\hline $308->314$ & 0.23024 & & & & \\
\hline $309->317$ & -0.10136 & & & & \\
\hline Excited State $17:$ & Singlet-AG & $2.9154 \mathrm{eV}$ & $425.28 \mathrm{~nm}$ & $\mathrm{f}=0.0000$ & $<\mathrm{S} * * 2>=0.000$ \\
\hline $307->312$ & 0.60342 & & & & \\
\hline $307->314$ & -0.23579 & & & & \\
\hline $308->313$ & -0.22912 & & & & \\
\hline $309->318$ & -0.10116 & & & & \\
\hline Excited State $18:$ & Singlet-AG & $2.9486 \mathrm{eV}$ & $420.48 \mathrm{~nm}$ & $\mathrm{f}=0.0000$ & $<\mathrm{S} * * 2>=0.000$ \\
\hline $307->313$ & -0.23505 & & & & \\
\hline $308->314$ & -0.23633 & & & & \\
\hline $309->316$ & 0.61078 & & & & \\
\hline
\end{tabular}




$\begin{array}{cccccc}\text { Excited State 19: } & \text { Singlet-AG } & 2.9724 \mathrm{eV} & 417.12 \mathrm{~nm} & \mathrm{f}=0.0000 & <\mathrm{S} * * 2>=0.000 \\ 307->313 & -0.10482 & & & & \\ 308->314 & 0.10391 & & & & \\ 309->317 & 0.66575 & & & & \\ \text { Excited State 20: } & \text { Singlet-AG } & 2.9727 \mathrm{eV} & 417.08 \mathrm{~nm} & \mathrm{f}=0.0000 & <\mathrm{S} * * 2>=0.000 \\ 307->314 & -0.10486 & & & & \\ 308->313 & -0.10338 & & & & \\ 309->318 & 0.66581 & & & & \end{array}$

$\underline{\text { Ridical cation } \mathbf{2 - \mathbf { H } ^ { + }}}$

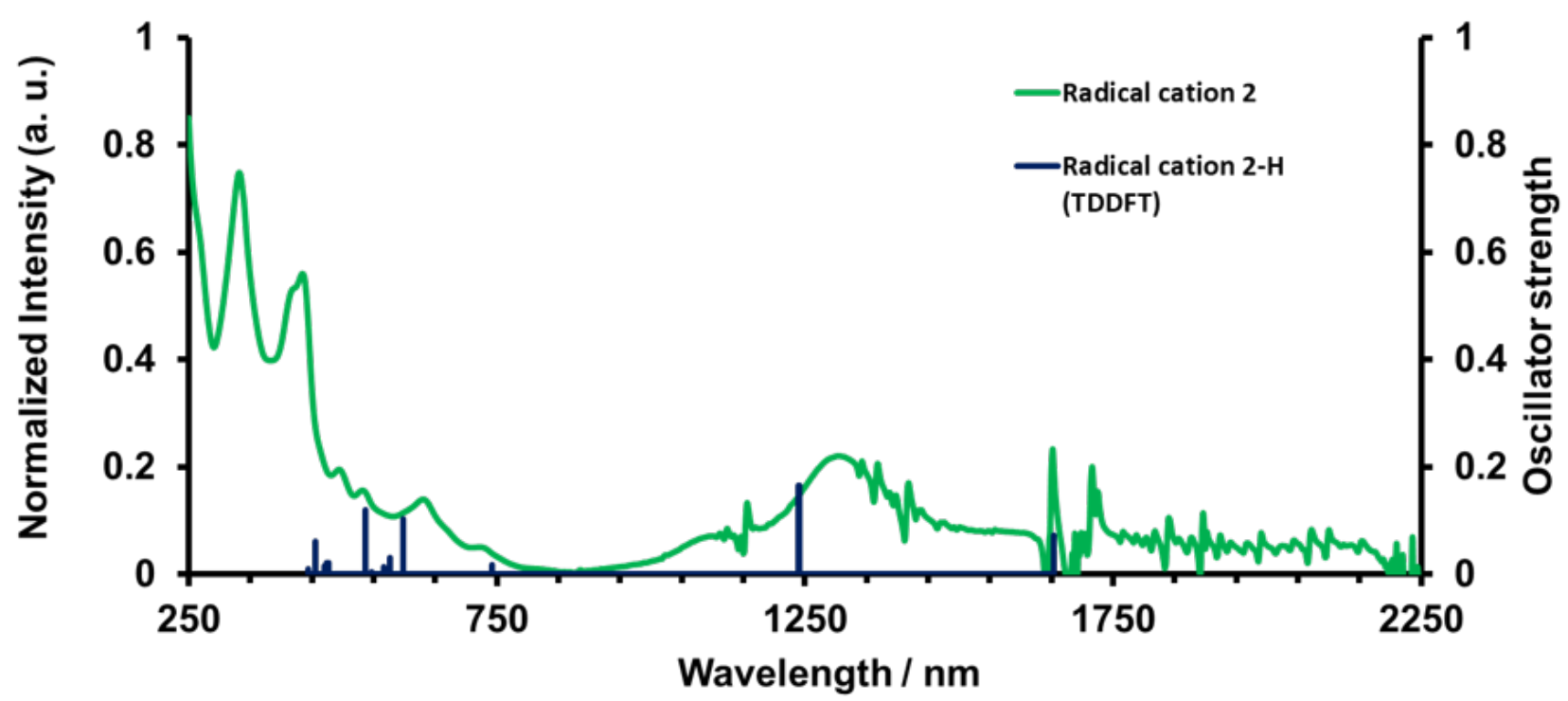

Figure S11b. UV-Vis-NIR spectra of $\mathbf{2}^{\cdot+}$ (green line) in $\mathrm{CH}_{2} \mathrm{Cl}_{2}$ and TD-DFT calculation of $\mathbf{2}-\mathbf{H}^{++}$(blue line).

Table S9. TD-DFT calculation result of $\mathbf{2}-\mathbf{H}^{++}$.

$\mathrm{HOMO}=308, \mathrm{SOMO}=309, \mathrm{LUMO}=310$

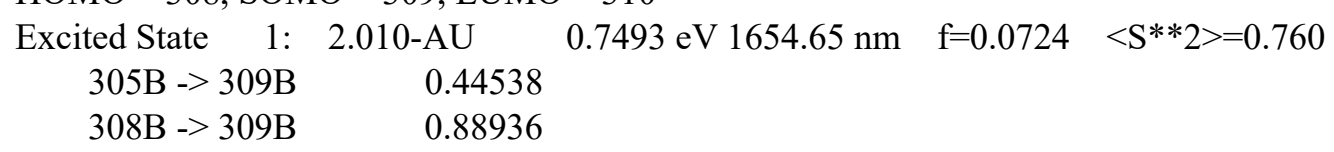

This state for optimization and/or second-order correction.

Total Energy, E(TD-HF/TD-KS) $=-3779.65539910$

Copying the excited state density for this state as the 1-particle RhoCI density.

$\begin{array}{crrrrr}\text { Excited State 2: } & 2.010-\mathrm{AU} & 0.7499 \mathrm{eV} 1653.28 \mathrm{~nm} & \mathrm{f}=0.0723 & <\mathrm{S}^{* *} 2>=0.760 \\ \text { 306B }->\text { 309B } & 0.44713 & & & \\ \text { 307B -> 309B } & 0.88847 & & & & \\ \text { Excited State 3: } & 2.022-\mathrm{AU} & 0.9993 \mathrm{eV} 1240.72 \mathrm{~nm} & \mathrm{f}=0.1660 & <\mathrm{S}^{* *} 2>=0.772 \\ \text { 306B -> 309B } & 0.88817 & & & & \\ \text { 307B -> 309B } & -0.43945 & & & & \\ \text { Excited State 4: } & 2.022-\mathrm{AU} & 0.9996 \mathrm{eV} 1240.30 \mathrm{~nm} & \mathrm{f}=0.1655 & <\mathrm{S}^{* *} 2>=0.772 \\ \text { 305B -> 309B } & 0.88904 & & & & \\ \text { 308B -> 309B } & -0.43771 & & & & \\ \text { Excited State 5: } & 2.032-\mathrm{AG} & 1.4824 \mathrm{eV} & 836.35 \mathrm{~nm} & \mathrm{f}=0.0000 & <\mathrm{S}^{* *} 2>=0.782 \\ \text { 302B -> 309B } & 0.98997 & & & & \\ \text { Excited State 6: } & 2.056-\mathrm{AG} & 1.5586 \mathrm{eV} & 795.46 \mathrm{~nm} & \mathrm{f}=0.0000 & <\mathrm{S}^{* *} 2>=0.807 \\ \text { 309A -> 311A } & -0.14728 & & & & \end{array}$




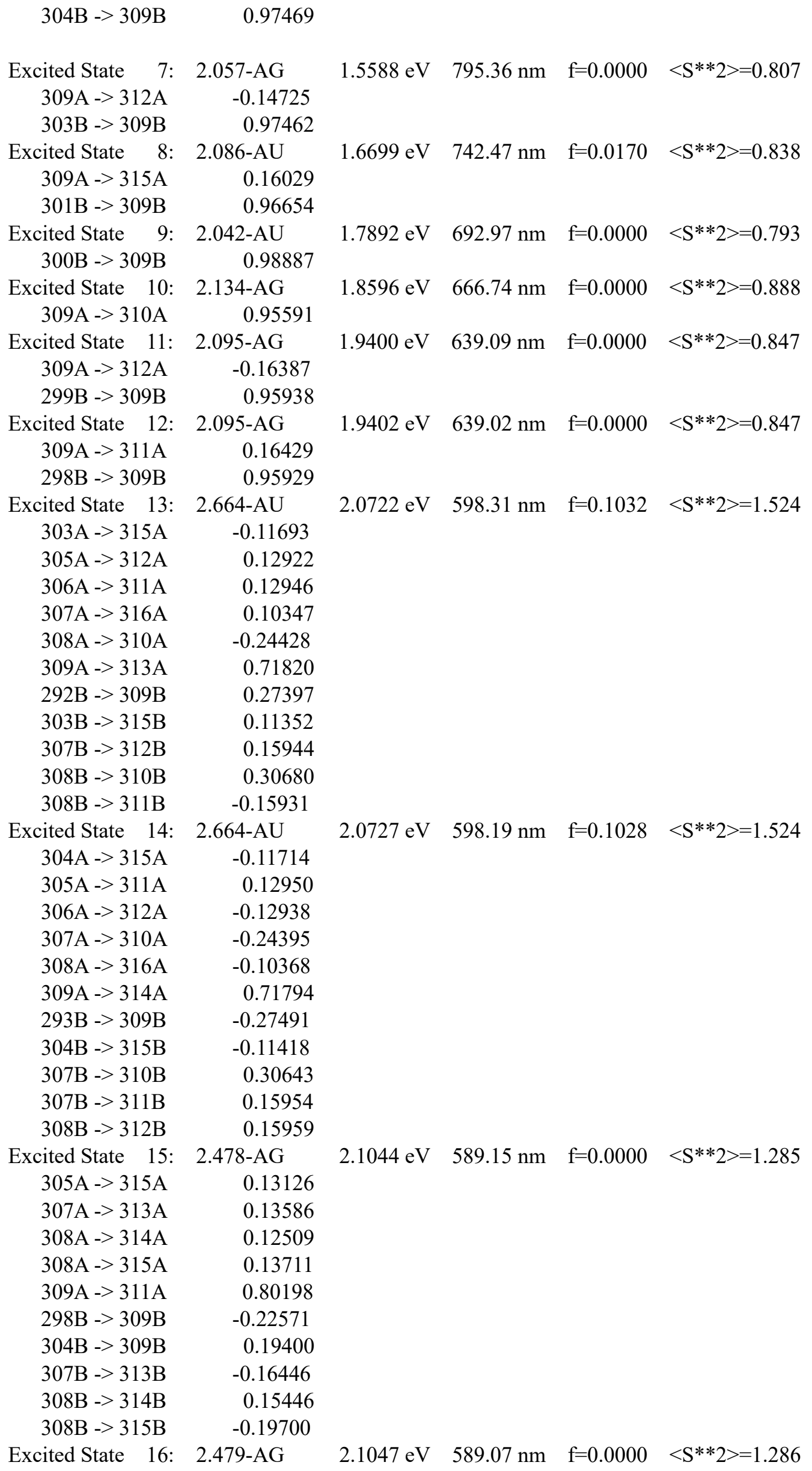

Excited State 16: $2.479-\mathrm{AG} \quad 2.1047 \mathrm{eV} \quad 589.07 \mathrm{~nm} \quad \mathrm{f}=0.0000 \quad<\mathrm{S} * * 2>=1.286$ 


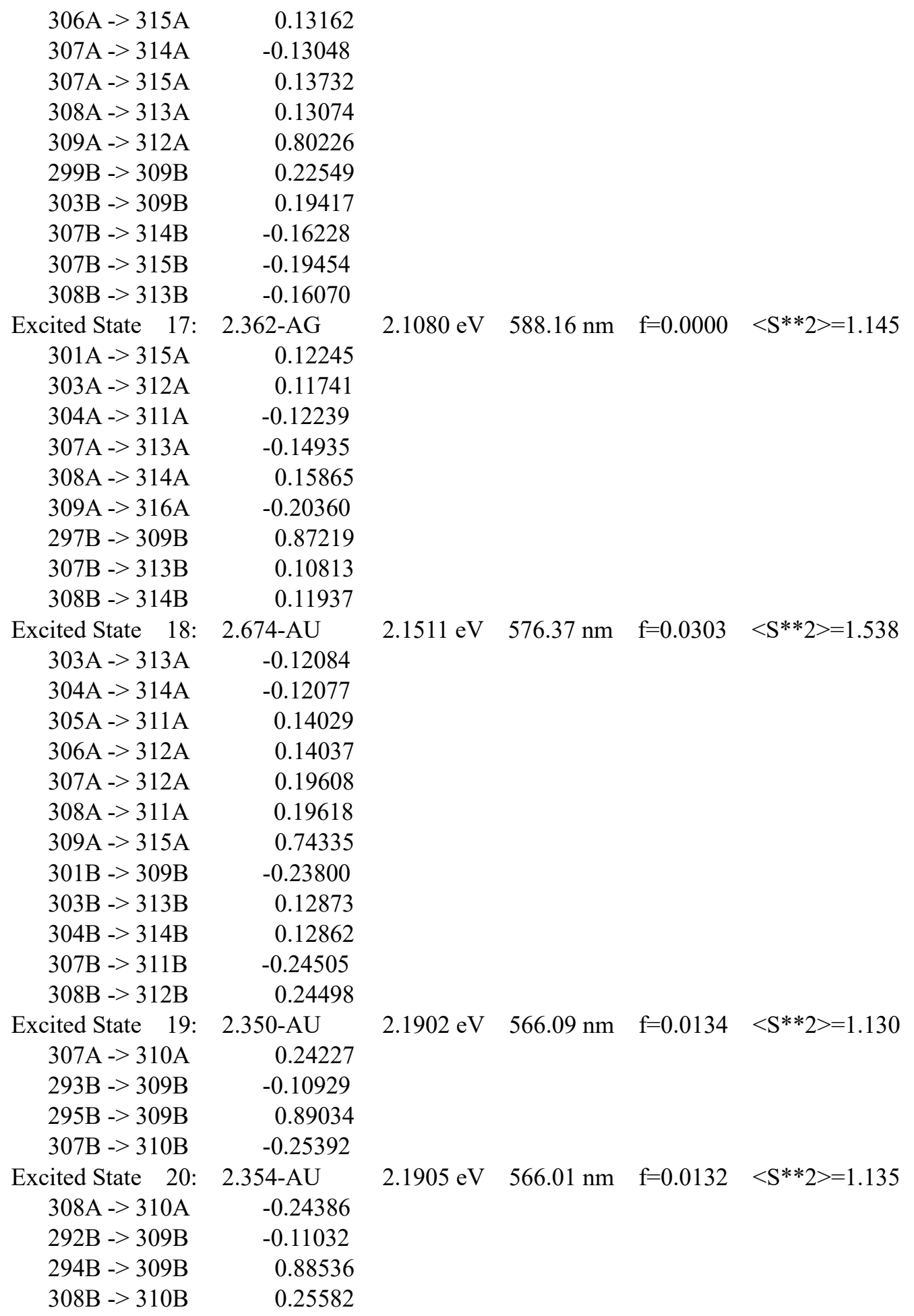




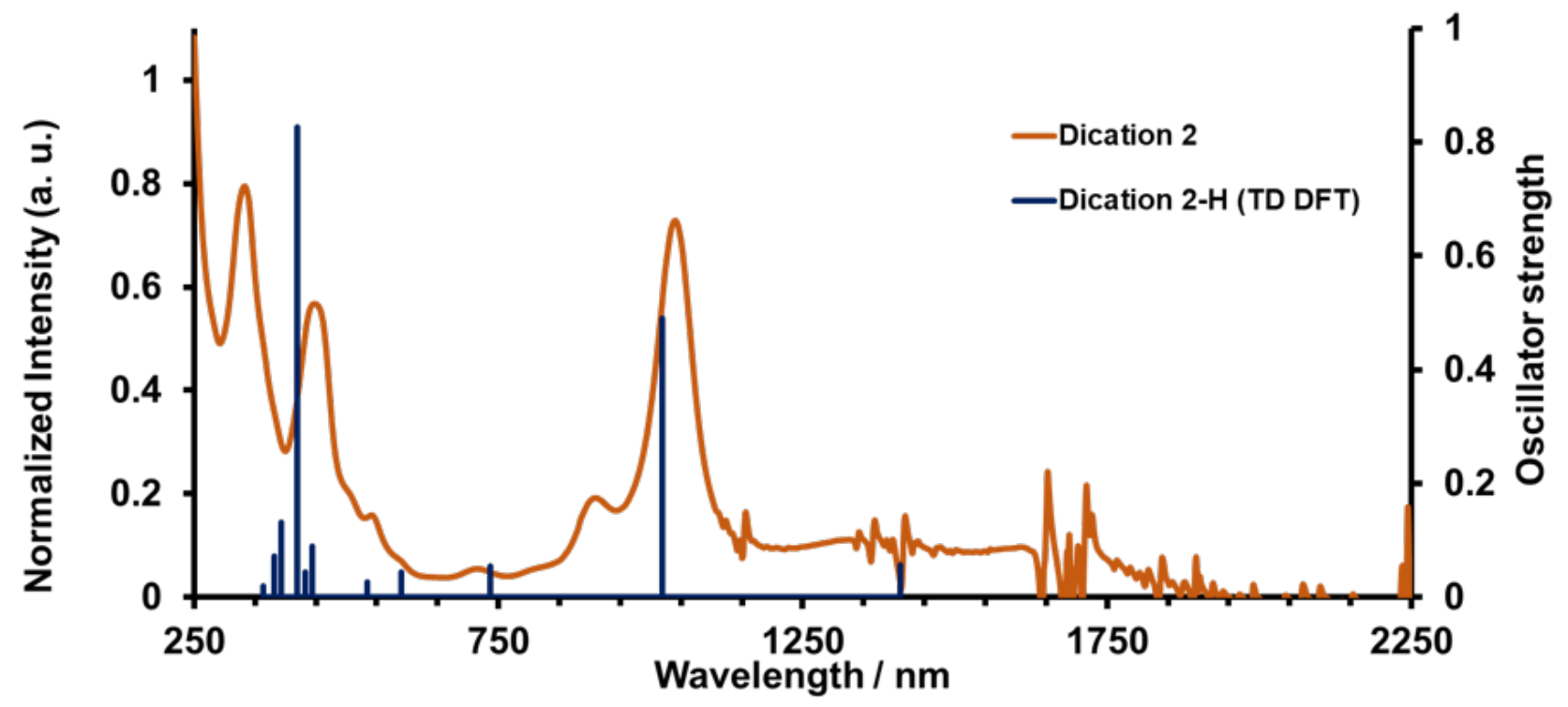

Figure S11c. UV-Vis-NIR spectra of $\mathbf{2}^{\mathbf{2}}$ (orange line) in $\mathrm{CH}_{2} \mathrm{Cl}_{2}$ and TD-DFT calculation of $\mathbf{2}-\mathbf{H}^{2+}$ (blue line).

Table S10. TD-DFT calculation result of 2-H ${ }^{2+}$.

$\mathrm{HOMO}=308, \mathrm{LUMO}=309$

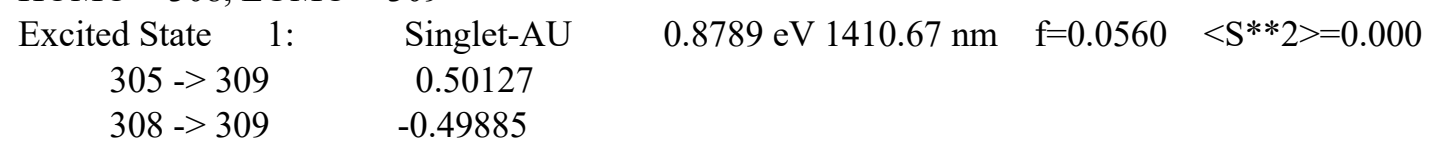

This state for optimization and/or second-order correction.

Total Energy, E(TD-HF/TD-KS) $=-3779.38698188$

Copying the excited state density for this state as the 1-particle RhoCI density.

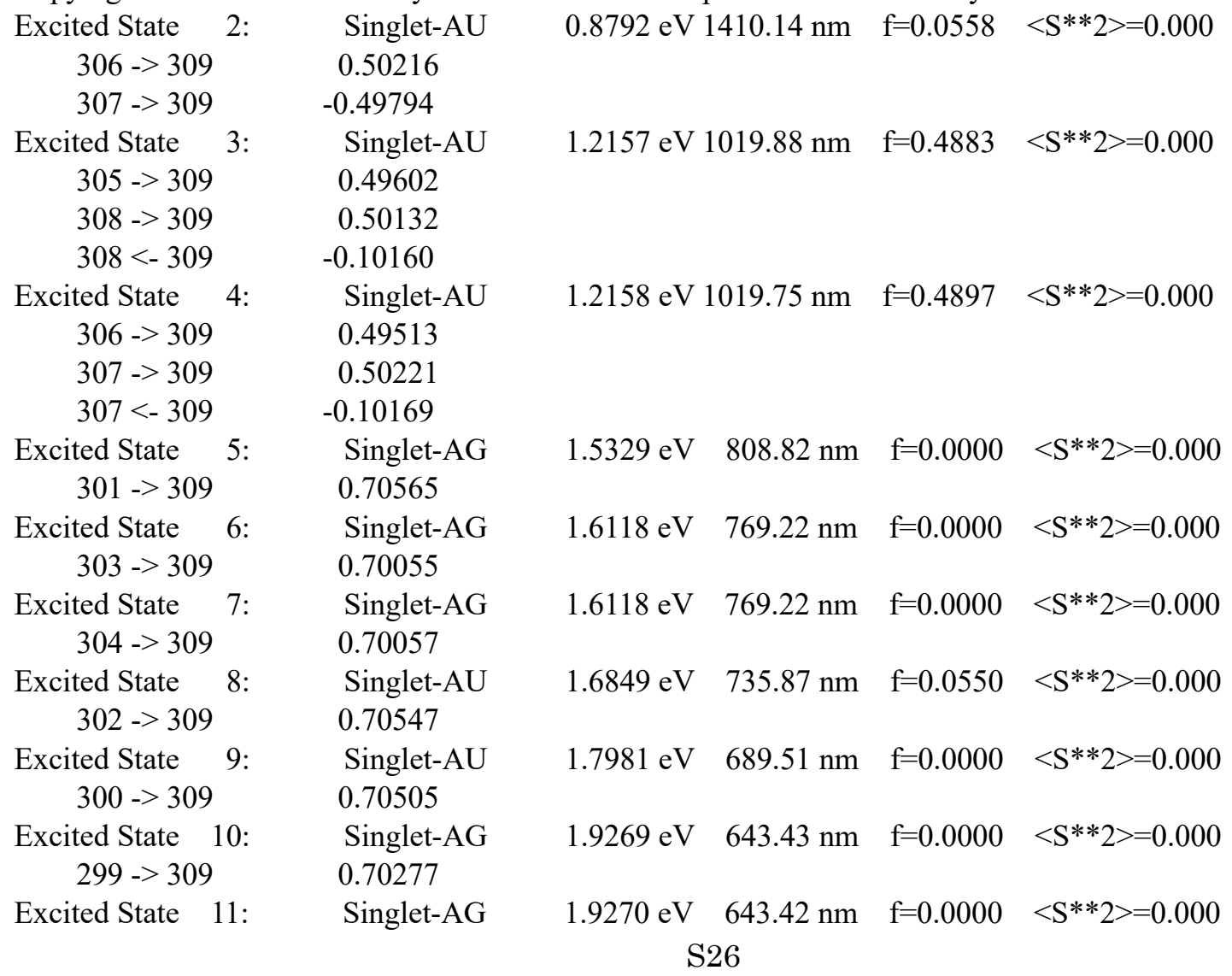


298 -> $309 \quad 0.70278$

\begin{tabular}{|c|c|c|c|c|c|}
\hline $\begin{array}{c}\text { Excited State 12: } \\
297-309\end{array}$ & Singlet-AG & $2.0906 \mathrm{eV}$ & $593.06 \mathrm{~nm}$ & $f=0.0000$ & $<\mathrm{S} * * 2>=0.000$ \\
\hline $\begin{array}{c}\text { Excited State 13: } \\
\quad 295->309\end{array}$ & $\begin{array}{l}\text { Singlet-AU } \\
0.70409\end{array}$ & $2.1007 \mathrm{eV}$ & $590.20 \mathrm{~nm}$ & $\mathrm{f}=0.0450$ & $<\mathrm{S} * * 2>=0.000$ \\
\hline $\begin{array}{c}\text { Excited State 14: } \\
294->309\end{array}$ & $\begin{array}{l}\text { Singlet-AU } \\
0.70409\end{array}$ & $2.1013 \mathrm{eV}$ & $590.03 \mathrm{~nm}$ & $\mathrm{f}=0.0450$ & $<\mathrm{S}^{* * 2}>=0.000$ \\
\hline $\begin{array}{l}\text { Excited State 15: } \\
\qquad 296->309\end{array}$ & $\begin{array}{l}\text { Singlet-AG } \\
0.69482\end{array}$ & $2.1859 \mathrm{eV}$ & $567.20 \mathrm{~nm}$ & $f=0.0000$ & $<S * * 2>=0.000$ \\
\hline $\begin{array}{c}\text { Excited State 16: } \\
\qquad 293->309\end{array}$ & $\begin{array}{l}\text { Singlet-AG } \\
0.70401\end{array}$ & $2.2822 \mathrm{eV}$ & $543.27 \mathrm{~nm}$ & $\mathrm{f}=0.0000$ & $<\mathrm{S}^{* * 2}>=0.000$ \\
\hline $\begin{array}{c}\text { Excited State 17: } \\
292->309\end{array}$ & $\begin{array}{l}\text { Singlet-AG } \\
0.70402\end{array}$ & $2.2832 \mathrm{eV}$ & $543.03 \mathrm{~nm}$ & $f=0.0000$ & $<\mathrm{S} * * 2>=0.000$ \\
\hline $\begin{array}{c}\text { Excited State 18: } \\
291->309\end{array}$ & $\begin{array}{l}\text { Singlet-AU } \\
0.70453\end{array}$ & $2.2872 \mathrm{eV}$ & $542.09 \mathrm{~nm}$ & $\mathrm{f}=0.0000$ & $<\mathrm{S}^{* * 2}>=0.000$ \\
\hline $\begin{array}{c}\text { Excited State 19: } \\
290->309 \\
307->310\end{array}$ & $\begin{array}{l}\text { Singlet-AU } \\
0.63124 \\
0.30848\end{array}$ & $2.3209 \mathrm{eV}$ & $534.20 \mathrm{~nm}$ & $f=0.0274$ & $<\mathrm{S}^{* * 2}>=0.000$ \\
\hline $\begin{array}{c}\text { Excited State 20: } \\
289->309 \\
308->310\end{array}$ & $\begin{array}{l}\text { Singlet-AU } \\
0.63072 \\
-0.30957\end{array}$ & $2.3211 \mathrm{eV}$ & $534.17 \mathrm{~nm}$ & $\mathrm{f}=0.0275$ & $<\mathrm{S}^{* * 2}>=0.000$ \\
\hline
\end{tabular}




\section{Titration experiments of 2 with fullerene, $C_{60}$}

\section{${ }^{1}$ H-NMR measurement:}

A mixture of HPHAC $2\left(5.76 \times 10^{-4} \mathrm{M}\right)$ and $\mathrm{C}_{60}\left(2.31 \times 10^{-3} \mathrm{M}\right)$ in toluene- $d_{8}$ was added stepwisely to the solution of HPHAC $2\left(5.76 \times 10^{-4} \mathrm{M}\right)$. The $1: 1$ complex formation was revealed by the continuous variation method as shown in Figure 4a.

\section{UV-vis absorption measurement:}

A mixture of HPHAC $2\left(1.28 \times 10^{-5} \mathrm{M}\right)$ and $\mathrm{C}_{60}\left(1.25 \times 10^{-3} \mathrm{M}\right)$ in toluene was added stepwisely to the solution of HPHAC $2\left(1.28 \times 10^{-5} \mathrm{M}\right)$. The stoichiometry and the association constant $\left(K_{\mathrm{a}}\right)$ were evaluated from the absorption spectra at $546 \mathrm{~nm}$ (Figure S12a). Chlorobenzene solution of $\mathrm{C}_{60}\left(5.83 \times 10^{-3} \mathrm{M}\right)$ was added stepwisely to solutions of HPHAC $\mathbf{2}, \mathbf{2}^{\cdot+}$ and $\mathbf{2}^{2+}$, respectively. The $K_{\mathrm{a}}$ were evaluated from the absorption spectra at 472, 1316 and 1042 $\mathrm{nm}$, respectively. (Figure $\mathrm{S} 12 \mathrm{~b}, \mathrm{c}$ ). The $K_{\mathrm{a}}$ was estimated by using a nonlinear curve-fitting method with the equation of $1: 1$ model as follows:

$\Delta \mathrm{A}=\left[\Delta \mathrm{A}_{\max }\left(1+K[\mathrm{G}]_{0}+K[\mathrm{H}]_{0}\right)-\left\{\Delta \mathrm{A}_{\max }^{2}\left(1+K[\mathrm{G}]_{0}+K[\mathrm{H}]_{0}\right)^{2}-4 K^{2}[\mathrm{G}]_{0}[\mathrm{H}]_{0} \Delta \mathrm{A}_{\max }^{2}\right\}^{1 / 2}\right] / 2 K[\mathrm{H}]_{0}$ $\Delta \mathrm{A}_{\max }=\left(\mathrm{A}_{\text {complex }}-\mathrm{A}_{\text {free }}\right)$

Table S11. Concentrations of $\mathrm{C}_{60}$ and chemical shift variations

\begin{tabular}{|c|c|c|}
\hline $\begin{array}{c}\mathrm{C}_{60}\left(10^{-4} \mathrm{M}\right) \\
\left(\text { Equivalent of } \mathrm{C}_{60}\right)\end{array}$ & $\Delta \delta\left(\mathrm{H}^{\mathrm{A}}, \mathrm{ppm}\right)$ & $\Delta \delta\left(\mathrm{H}^{\mathrm{B}}, \mathrm{ppm}\right)$ \\
\hline $0(0)$ & $0(8.225)$ & $0(7.879)$ \\
$1.61(0.28)$ & 0.072 & 0.033 \\
$3.01(0.52)$ & 0.127 & 0.059 \\
$4.24(0.74)$ & 0.165 & 0.076 \\
$5.98(1.04)$ & 0.199 & 0.091 \\
$7.16(1.24)$ & 0.209 & 0.096 \\
$8.66(1.50)$ & 0.216 & 0.099 \\
$10.08(1.75)$ & 0.22 & 0.101 \\
$11.54(2.00)$ & 0.223 & 0.102 \\
$12.94(2.25)$ & 0.225 & 0.103 \\
$14.37(2.54)$ & 0.226 & 0.103 \\
$15.87(2.75)$ & 0.226 & 0.104 \\
$17.31(3.00)$ & 0.227 & 0.104 \\
\hline
\end{tabular}



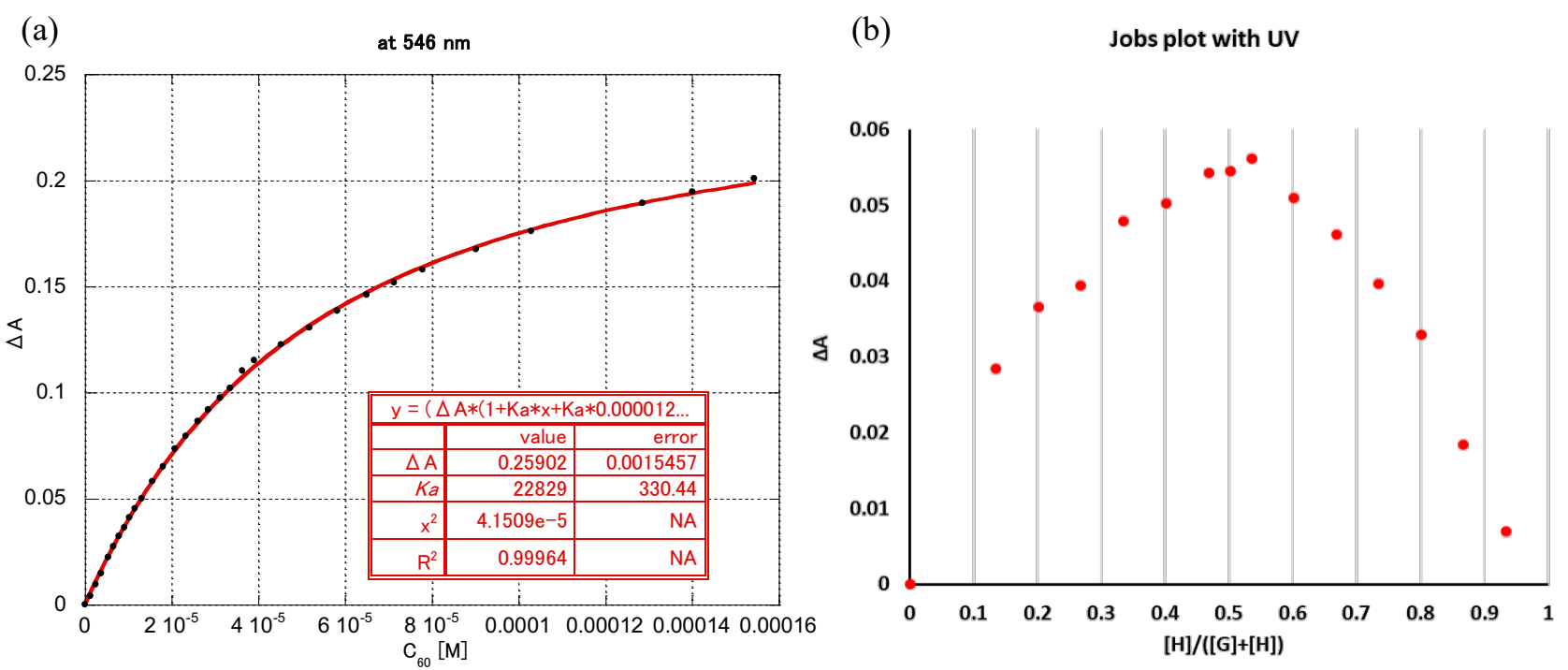

(c)

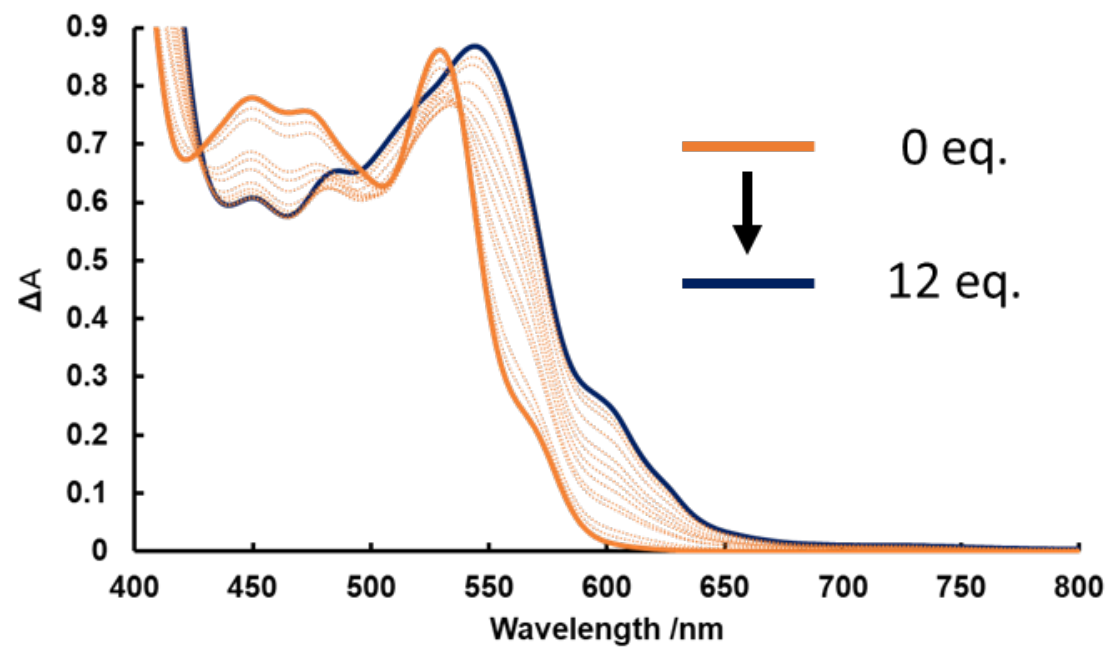

Figure S12a. (a) Nonlinear curve regression analysis of 2 with the titration of $\mathrm{C}_{60}(0-12$ eq.), (b) Job's plot with monitoring the absorption at $546 \mathrm{~nm}\left([\mathrm{H}, 2]=1.3 \times 10^{-4} \mathrm{M}\right.$ and $\left.\left[\mathrm{G}, \mathrm{C}_{60}\right]=1.3 \times 10^{-4} \mathrm{M},[\mathrm{H}]+[\mathrm{G}]=1.3 \times 10^{-4} \mathrm{M}\right)$, and (c) spectral changes in the absorption spectra of 2 upon addition of $\mathrm{C}_{60}\left([2]=1.28 \times 10^{-5} \mathrm{M}\right.$ in toluene). 
(a)

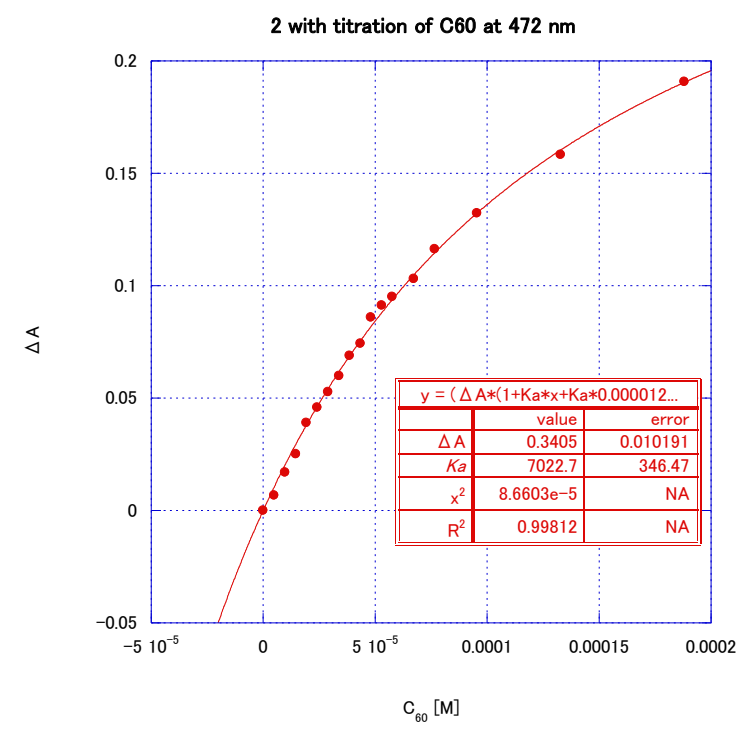

(b)

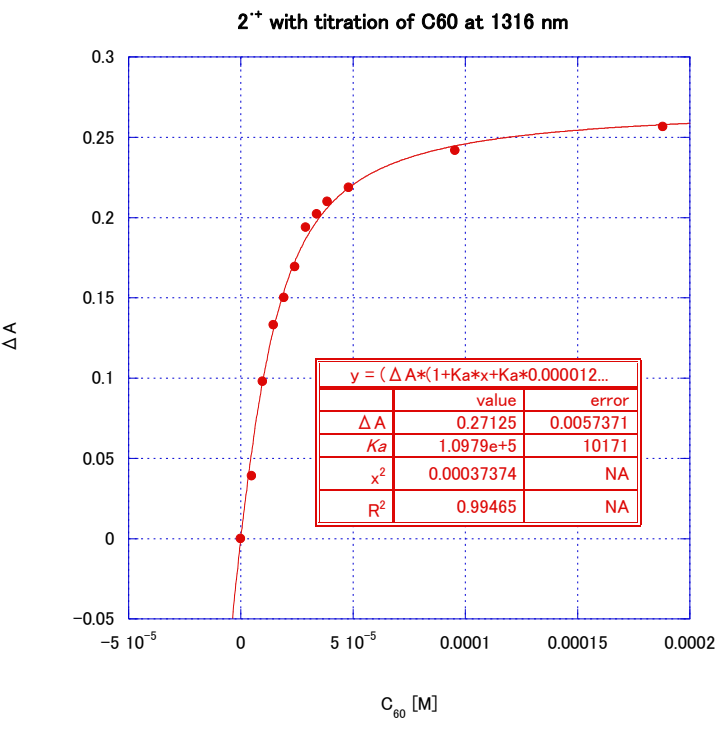

(c)

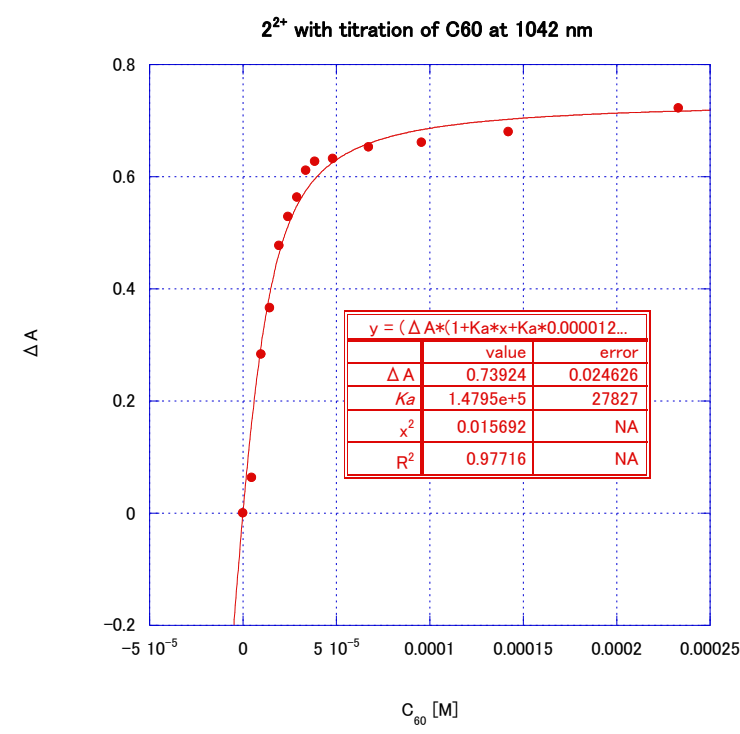

Figure S12b. Nonlinear curve regression analysis of (a) $\mathbf{2}$, (b) $\mathbf{2}^{\cdot+}$ and (c) $\mathbf{2}^{2+}$ with the titration of $\mathrm{C}_{60}\left(\left[\mathbf{2}, \mathbf{2}^{\mathbf{*}}\right.\right.$, and $\left.\mathbf{2}^{2+}\right]=1.28 \times 10^{-5} \mathrm{M}$ in chlorobenzene). 
(a)

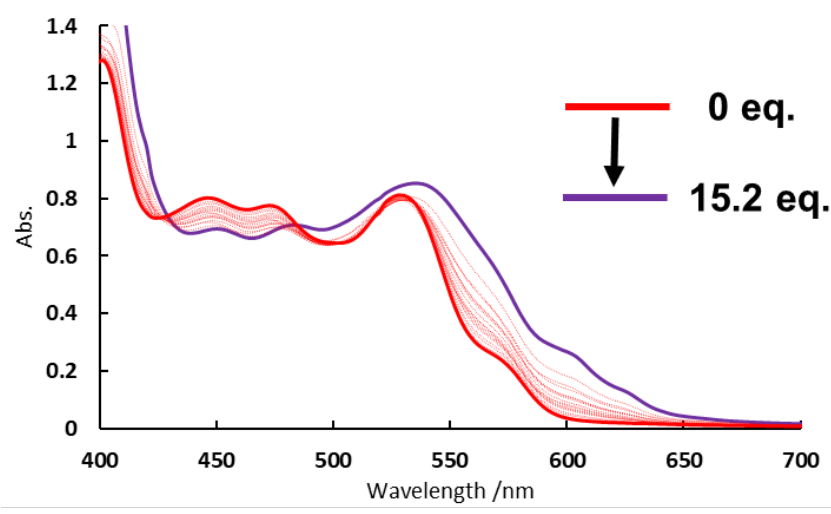

(b)

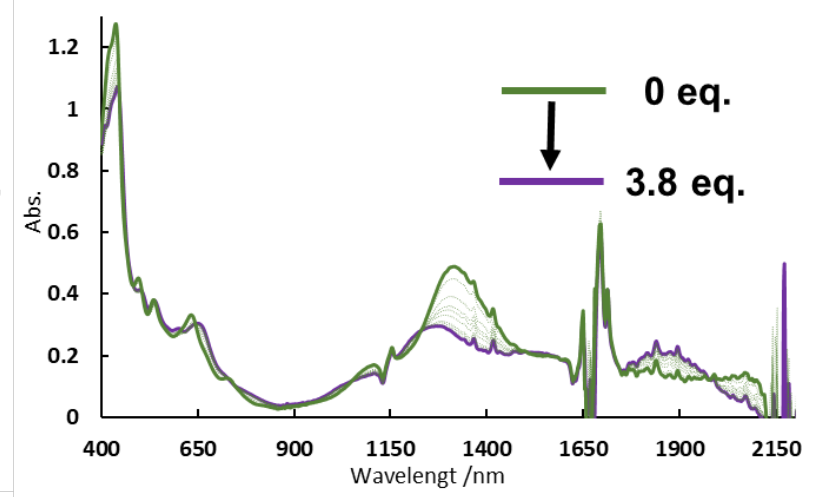

(c)

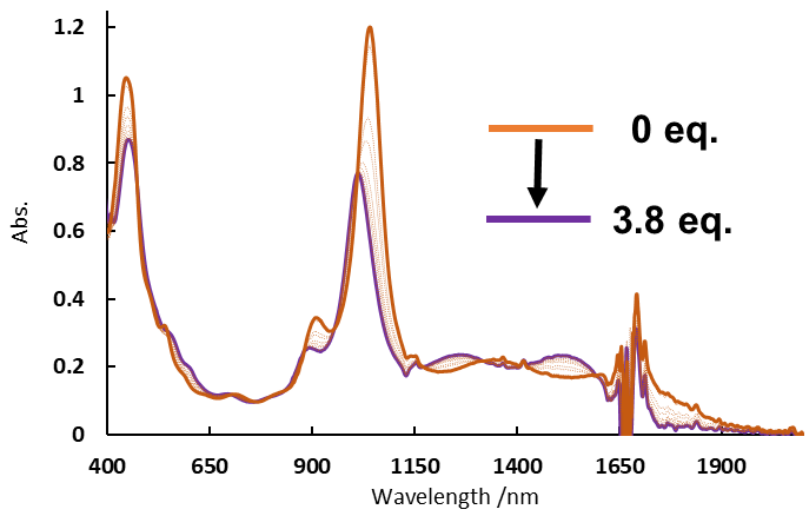

Figure S12c. Spectral changes in the absorption spectra of (a) 2, (b) $\mathbf{2}^{\cdot+}$ and (c) $\mathbf{2}^{2+}$ upon addition of $\mathrm{C}_{60}\left(\left[\mathbf{2}, \mathbf{2}^{\cdot+}\right.\right.$, and $\left.\mathbf{2}^{2+}\right]=1.28 \times 10^{-5} \mathrm{M}$ in chlorobenzene). 
13. MCD spectra of 1a and 2

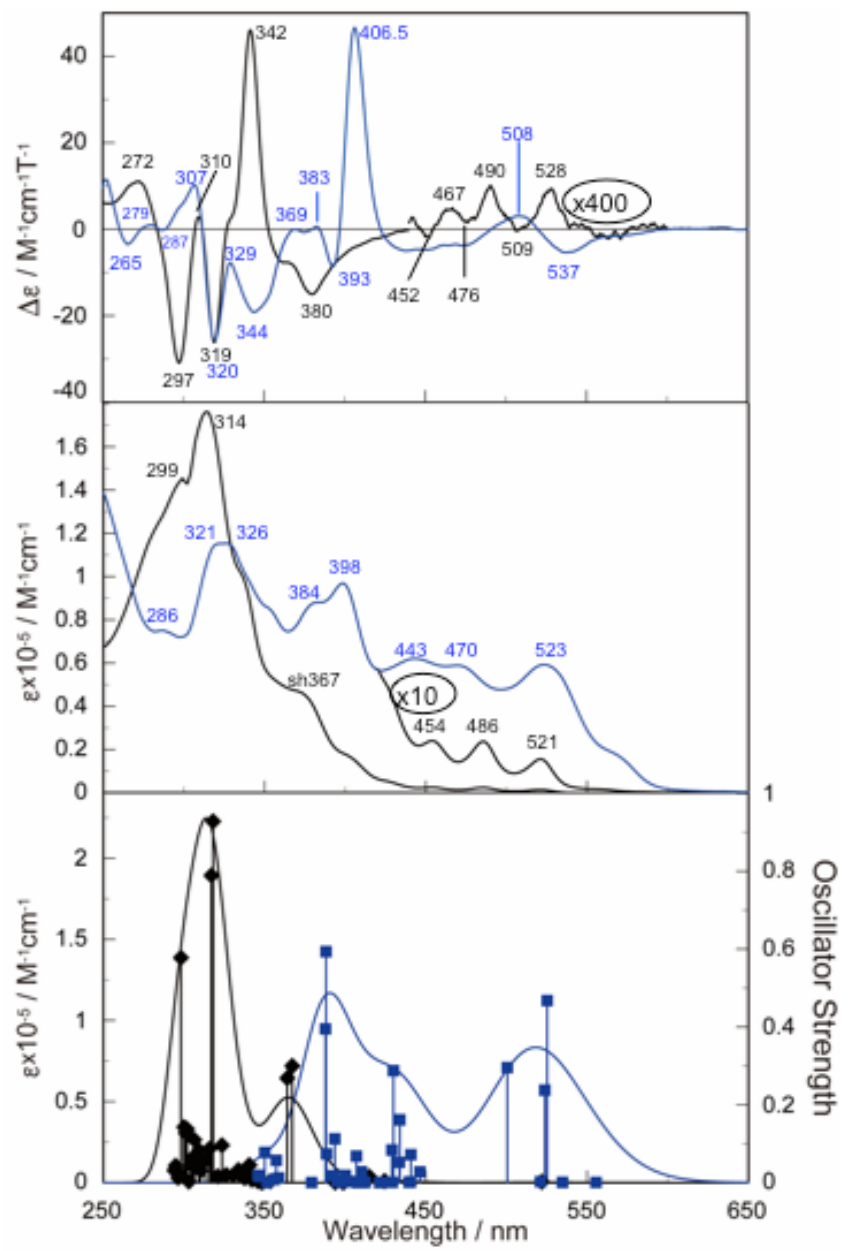

Figure S13. MCD (top) and UV-vis (middle) spectra in $\mathrm{CH}_{2} \mathrm{Cl}_{2}$ and TD-DFT calculation results (bottom) of 1a (black) and 2 (blue). 
Table S12. Calculated transition energies, oscillator strength $(f)$, assignment, and composition for $1 a^{a}{ }^{a}$

\begin{tabular}{|c|c|c|c|c|}
\hline Energy/eV (nm) & $f$ & $\mathrm{MCD}^{b)}$ & Assign. & Composition (weight \%) \\
\hline $2.376(521.9)$ & 0.0021 & & S & $\mathrm{H}\left(s^{-}\right) \rightarrow \mathrm{L}\left(s^{+}\right)(99.0 \%)$ \\
\hline $3.377(367.2)$ & 0.2995 & -(ca. 380) & $\mathrm{P} 1\left(+\mathrm{N}_{1}\right)$ & $\mathrm{H}(s-) \rightarrow \mathrm{L}+3(l-)(83.9), \mathrm{H}-1(h+) \rightarrow \mathrm{L}(s+)(11.9)$ \\
\hline $3.406(364.1)$ & 0.2684 & $-($ ca. 380$)$ & $\mathrm{P}_{2}\left(+\mathrm{N}_{2}\right)$ & $\mathrm{H}(s-) \rightarrow \mathrm{L}+4(l+)(83.8), \mathrm{H}-2(h+) \rightarrow \mathrm{L}(s+)(11.3)$ \\
\hline $3.832(323.5)$ & 0.0958 & & $\mathrm{CT}_{1}$ & $\mathrm{H}\left(s_{-}\right) \rightarrow \mathrm{L}+12(69.6), \mathrm{H}\left(s_{-}\right) \rightarrow \mathrm{L}+13(10.8)$ \\
\hline $3.899(318.0)$ & 0.9282 & $+(342)$ & $\begin{array}{l}\pi \pi *(+\mathrm{CT}) \\
\left(+\mathrm{N}_{2}\right)\end{array}$ & $\begin{array}{l}\mathrm{H}-1(h+) \rightarrow \mathrm{L}+2(l-)(15.1), \mathrm{H}-4(h-) \rightarrow \mathrm{L}(s+)(12.8), \\
\mathrm{H}-2(h+) \rightarrow \mathrm{L}+2(l-)(11.2), \mathrm{H}-3(h-) \rightarrow \mathrm{L}(s+)(9.9), \\
\mathrm{H}-1(h+) \rightarrow \mathrm{L}+1(l-)(6.0), \mathrm{H}-3(h-) \rightarrow \mathrm{L}+2(l-)(9.0), \\
\mathrm{H}(s-) \rightarrow \mathrm{L}+14(6.2), \mathrm{H}-5(h-) \rightarrow \mathrm{L}(s+)(3.4)\end{array}$ \\
\hline $3.911(317.0)$ & 0.7892 & $-(319)$ & $\begin{array}{l}\pi \pi *(+\mathrm{CT}) \\
\left(+\mathrm{N}_{2}\right)\end{array}$ & $\begin{array}{l}\mathrm{H}-2(h+) \rightarrow \mathrm{L}+2(l-)(14.4), \mathrm{H}-4(h-) \rightarrow \mathrm{L}(s+)(11.0), \\
\mathrm{H}-1(h+) \rightarrow \mathrm{L}+1(l-)(9.9), \mathrm{H}-3(h-) \rightarrow \mathrm{L}(s+)(9.1), \\
\mathrm{H}(s-) \rightarrow \mathrm{L}+15(8.0), \mathrm{H}-1(h+) \rightarrow \mathrm{L}+2(l-)(6.8), \\
\mathrm{H}(s-) \rightarrow \mathrm{L}+12(5.6), \mathrm{H}-2(h+) \rightarrow \mathrm{L}+1(l-)(5.1), \\
\mathrm{H}(s-) \rightarrow \mathrm{L}+1(l-)(5.1), \mathrm{H}-5 \rightarrow \mathrm{L}(s+)(4.9), \mathrm{H}(s-) \rightarrow \mathrm{L}+16(3.9), \\
\mathrm{H}-4(h-) \rightarrow \mathrm{L}+1(l-)(3.4)\end{array}$ \\
\hline $3.928(315 \cdot 7)$ & 0.0865 & & $\pi \pi *(+\mathrm{CT})$ & $\mathrm{H}_{-5} \rightarrow \mathrm{L}\left(s^{+}\right)(70.5), \mathrm{H}\left(s^{-}\right) \rightarrow \mathrm{L}+14(6.1), \mathrm{H}\left(s^{-}\right) \rightarrow \mathrm{L}+12(4.4)$ \\
\hline $4.010(309.2)$ & 0.0836 & & $\pi \pi *(+\mathrm{CT})$ & $\mathrm{H}\left(s^{-}\right) \rightarrow \mathrm{L}+15(70.4), \mathrm{H}\left(s^{-}\right) \rightarrow \mathrm{L}+16(8.9), \mathrm{H}\left(s^{-}\right) \rightarrow \mathrm{L}+17$ (6.1) \\
\hline $4.047(306.4)$ & 0.1100 & & & $\begin{array}{l}\mathrm{H}-2(h+) \rightarrow \mathrm{L}+3(l+)(22.5), \mathrm{H}-1(h+) \rightarrow \mathrm{L}+4(1+)(18.3) \\
\mathrm{H}(s-) \rightarrow \mathrm{L}+16(16.9), \mathrm{H}(s-) \rightarrow \mathrm{L}+17(16.6)\end{array}$ \\
\hline 4.108 (301.9) & 0.1373 & & & $\begin{array}{l}\mathrm{H}-3(h-) \rightarrow \mathrm{L}+1(l-)(51.0), \mathrm{H}(s-) \rightarrow \mathrm{L}+19(20.2) \\
\mathrm{H}-2(l+) \rightarrow \mathrm{L}+4(l+)(18.3)\end{array}$ \\
\hline 4.119 (301.0) & 0.1277 & & & $\begin{array}{l}\mathrm{H}(s-) \rightarrow \mathrm{L}+19(66.6), \mathrm{H}-3(h-) \rightarrow \mathrm{L}+1(l-)(10.3), \\
\mathrm{H}(s-) \rightarrow \mathrm{L}+2 \mathrm{O}(6.6)\end{array}$ \\
\hline $4.132(300.1)$ & 0.1422 & & & $\begin{array}{l}\mathrm{H}(s-) \rightarrow \mathrm{L}+2 \mathrm{O}(68.3), \mathrm{H}-3(h-) \rightarrow \mathrm{L}+1(l-)(9.7), \\
\mathrm{H}-4(h-) \rightarrow \mathrm{L}+1(l-)(5.6)\end{array}$ \\
\hline $4.160(298.1)$ & 0.5779 & $-(297)$ & $\mathrm{CT}_{1}$ & $\begin{array}{l}\mathrm{H}-4(h-) \rightarrow \mathrm{L}+1(l-)(40.5), \mathrm{H}-3(h-) \rightarrow \mathrm{L}+2(l-)(25.8), \\
\mathrm{H}(s-) \rightarrow \mathrm{L}+2 \mathrm{O}(5.9), \mathrm{H}\left(s_{-}\right) \rightarrow \mathrm{L}+27 \text { (3.0) }\end{array}$ \\
\hline
\end{tabular}

\footnotetext{
a) Except the transition at $2.376 \mathrm{eV}$, transitions with oscillator strength $\boldsymbol{f}$ greater than 0.07 and contribution (weight \%) larger than $3 \%$ are collected. Note in this molecule, $\mathrm{H}-1$ and $\mathrm{H}-2, \mathrm{H}-3$ and $\mathrm{H}-4, \mathrm{~L}+1$ and $\mathrm{L}+2$, and $\mathrm{L}+3$ and $\mathrm{L}+4$ are each nearly doubly degenerate. Although $\mathrm{L}+1 \sim \mathrm{L}+4$ orbitals have large coefficients in the outer $\mathrm{CF}_{3} \mathrm{Ph}$ moiety (Fig. S6a), we judged that $\mathrm{L}+1$ and $\mathrm{L}+2$ correspond to the $l$ - orbital while $\mathrm{L}+3$ and $\mathrm{l}+4$ are $l+$ orbital since they also have large coefficients in the inner perimeter. From the shape of MOs, the calculated L+3 (orbital No. 553) and L+4 (554) having seven nodal planes appear to correspond to spectroscopic L+1 (i.e. $l$-) and L+2 (i.e. $l+$ ) orbital, respectively. CT means charge-transfer from the inner perimeter including central benzene molecule to outer $\mathrm{CF}_{3} \mathrm{Ph}$ moiety, while $\mathrm{CT}_{1}$ is that from the inner perimeter without central benzene moiety to outer $\mathrm{CF}_{3} \mathrm{Ph}$ moiety. The contribution in parenthesis is smaller than that without it.
}

b) Observed MCD sign and wavelength. 
Table S13. Calculated transition energies, oscillator strength $(f)$, assignment, and composition for $\mathbf{2}^{a}{ }^{a}$

Energy/eV (nm) $\quad \boldsymbol{f} \quad \mathrm{MCD}^{b)} \quad$ Assign. Composition (weight \%)

\begin{tabular}{|c|c|c|c|c|}
\hline $2.360(525 \cdot 3)$ & 0.4402 & $-(537)$ & $\mathrm{CT}_{1}\left(+\mathrm{N}_{1}\right)$ & $\mathrm{H}(s-) \rightarrow \mathrm{L}+3(94.0), \mathrm{H}-1(h+) \rightarrow \mathrm{L}+5(s+)(2.8)$ \\
\hline $2.361(525.1)$ & 0.4391 & $+(508)$ & $\mathrm{CT}_{1}\left(+\mathrm{N}_{1}\right)$ & $\mathrm{H}(s-) \rightarrow \mathrm{L}+4(94.0), \mathrm{H}-2(h+) \rightarrow \mathrm{L}+5(s+)(2.8)$ \\
\hline $2.371(522.9)$ & 0.0782 & - & $\mathrm{CT}_{1}$ & $\mathrm{H}(s-) \rightarrow \mathrm{L}+2(99.1)$ \\
\hline $2.854(434.4)$ & 0.3206 & - & $\mathrm{CT}\left(+\pi \pi^{*}\right)$ & $\begin{array}{l}\mathrm{H}-2(h+) \rightarrow \mathrm{L}+1(43.0), \mathrm{H}-1(h+) \rightarrow \mathrm{L}(40.5), \mathrm{H}-2(h+) \rightarrow \mathrm{L}(3.6), \\
\mathrm{H}-1(h+) \rightarrow \mathrm{L}+1(3.6), \mathrm{H}-3(h-) \rightarrow \mathrm{L}+5(s+)(3.1)\end{array}$ \\
\hline $2.854(434.3)$ & 0.3225 & - & $\mathrm{CT}\left(+\pi \pi^{*}\right)$ & $\begin{array}{l}\mathrm{H}-1(h+) \rightarrow \mathrm{L}+1(43.0), \mathrm{H}-2(h+) \rightarrow \mathrm{L}(41.3), \mathrm{H}-2(h+) \rightarrow \mathrm{L}+1(3.7), \\
\mathrm{H}-1(h+) \rightarrow \mathrm{L}(3.5)\end{array}$ \\
\hline $3.167(391.5)$ & 0.0793 & - & $\mathrm{CT}\left(+\pi \pi^{*}\right)$ & $\begin{array}{l}\mathrm{H}-3(h-) \rightarrow \mathrm{L}+1(45.2), \mathrm{H}-4(h-) \rightarrow \mathrm{L}(45.0), \mathrm{H}-4(h-) \rightarrow \mathrm{L}+1(3.6), \\
\mathrm{H}-3(h-) \rightarrow \mathrm{L}(3.6)\end{array}$ \\
\hline $3.212(386.1)$ & 0.5770 & $+(406.5)$ & $\mathrm{N}_{2}$ & $\mathrm{H}-3(h-) \rightarrow \mathrm{L}+5(s+)(81.8), \mathrm{H}-4(h-) \rightarrow \mathrm{L}+5(s+)(7.4)$ \\
\hline $3.212(386.0)$ & 0.5774 & $-(393)$ & $\mathrm{N}_{2}$ & $\mathrm{H}-4(h-) \rightarrow \mathrm{L}+5(s+)(81.8), \mathrm{H}-3(h-) \rightarrow \mathrm{L}+5(s+)(7.4)$ \\
\hline $3.600(344.4)$ & 0.0971 & & & $\begin{array}{l}\mathrm{H}-2(h+) \rightarrow \mathrm{L}+8(19.4), \mathrm{H}-1(h+) \rightarrow \mathrm{L}+7(19.1), \mathrm{H}-5 \rightarrow \mathrm{L}+3(18.8), \\
\mathrm{H}-4(h-) \rightarrow \mathrm{L}+6(10.1), \mathrm{H}(s-) \rightarrow \mathrm{L}+1 \mathrm{O}(l+)(4.6), \\
\mathrm{H}-1(h+) \rightarrow \mathrm{L}+5(s+)(4.6)\end{array}$ \\
\hline $3.601(344 \cdot 3)$ & 0.0969 & & & $\begin{array}{l}\mathrm{H}-2(h+) \rightarrow \mathrm{L}+7(19.3), \mathrm{H}-1(h+) \rightarrow \mathrm{L}+8(19.2), \mathrm{H}-5 \rightarrow \mathrm{L}+4(18.6) \\
\mathrm{H}-3(h-) \rightarrow \mathrm{L}+6(10.2), \mathrm{H}(s-) \rightarrow \mathrm{L}+9(l-)(4.7) \\
\mathrm{H}-2(h+) \rightarrow \mathrm{L}+6(4.6), \mathrm{H}-9 \rightarrow \mathrm{L}(3.5)\end{array}$ \\
\hline $3.701(335.1)$ & 0.2583 & $-(344)$ & $\mathrm{CT}\left(+\pi \pi^{*}\right)$ & $\begin{array}{l}\mathrm{H}-2(h+) \rightarrow \mathrm{L}+8(16.3), \mathrm{H}-1(h+) \rightarrow \mathrm{L}+7(16.1), \mathrm{H}-5 \rightarrow \mathrm{L}+3(15.2) \\
\mathrm{H}-1(h+) \rightarrow \mathrm{L}+6(13.9), \mathrm{H}-6 \rightarrow \mathrm{L}+2(8.8), \mathrm{H}-9 \rightarrow \mathrm{L}+1(5.6) \\
\mathrm{H}-8 \rightarrow \mathrm{L}(3.7), \mathrm{H}(s-) \rightarrow \mathrm{L}+10(l+)(3.0)\end{array}$ \\
\hline $3.701(335.0)$ & 0.2599 & $-(344)$ & $\mathrm{CT}\left(+\pi \pi^{*}\right)$ & $\begin{array}{l}\mathrm{H}-2(h+) \rightarrow \mathrm{L}+7(16.3), \mathrm{H}-1(h+) \rightarrow \mathrm{L}+8(16.0), \mathrm{H}-5 \rightarrow \mathrm{L}+4(14.8), \\
\mathrm{H}-2(h+) \rightarrow \mathrm{L}+6(13.9), \mathrm{H}-7 \rightarrow \mathrm{L}+2(8.9), \mathrm{H}-9 \rightarrow \mathrm{L}(5.8), \\
\mathrm{H}-8 \rightarrow \mathrm{L}+1(5.8), \mathrm{H}\left(s_{-}\right) \rightarrow \mathrm{L}+9(l-)(3.1)\end{array}$ \\
\hline
\end{tabular}

\footnotetext{
a) Except transitions at $2.360 \& 2.361 \mathrm{eV}$, transitions with oscillator strength $f$ greater than 0.07 and contribution (weight \%) larger than 3.0\% are collected. Note in this molecule, $\mathrm{H}-1$ and $\mathrm{H}-2$, and $\mathrm{H}-3$ and $\mathrm{H}-4$ are each doubly degenerate, and L+1 $\sim \mathrm{L}+4$ are practically all acenaphthene-centered orbitals although they have some coefficients along the inner perimeter (Fig. S6b). CT means charge-transfer from the inner perimeter including central benzene molecule to outer acenaphthene moiety, while $\mathrm{CT}_{1}$ is that from the inner perimeter without central benzene molecule to outer acenaphthene moiety. From the shape of MOs, the calculated L+9 (orbital No. 319) and L+10 (320) having seven nodal planes appear to correspond to spectroscopic L+1 (i.e. $l$-) and L+2 (i.e. $l+$ ) orbital, respectively. The contribution in parenthesis is smaller than that without it.
}

b) Observed MCD sign and wavelength. 


\section{Atomic coordinates of $2-\mathrm{H}, 2-\mathrm{H}^{++}$, and $2-\mathrm{H}^{2+}$}

The calculations were performed at the R(U)B3LYP/6-31G(d) level of theory.

\section{Neutral 2-H}

Total energy $=-3779.8824$ a.u., Imaginary frequency $=0$

\begin{tabular}{|c|c|c|c|c|}
\hline 70 & Symbol & $\mathrm{X}$ & $\mathrm{Y}$ & Z \\
\hline 1 & $\mathrm{C}$ & 1.1953040 & 0.6902300 & 0.0239120 \\
\hline$?$ & $\mathrm{C}$ & -0.0000110 & 1.3803640 & -0.0282920 \\
\hline & $\mathrm{C}$ & -1.1953320 & 0.6902560 & 0.0235350 \\
\hline & $\mathrm{C}$ & -1.1953040 & -0.6902300 & -0.0239120 \\
\hline & $\mathrm{C}$ & 0.0000110 & -1.3803640 & 0.0282920 \\
\hline & $\mathrm{C}$ & 1.1953320 & -0.6902560 & -0.0235350 \\
\hline & $\mathrm{N}$ & 2.3804480 & -1.3746890 & -0.0472480 \\
\hline & $\mathrm{N}$ & 2.3804270 & 1.3746490 & 0.0479890 \\
\hline & $\mathrm{N}$ & -0.0000290 & 2.7488770 & -0.0560600 \\
\hline & $\mathrm{N}$ & -2.3804480 & 1.3746890 & 0.0472480 \\
\hline & $\mathrm{N}$ & -2.3804270 & -1.3746490 & -0.0479890 \\
\hline & $\mathrm{N}$ & 0.0000290 & -2.7488770 & 0.0560600 \\
\hline & $\mathrm{C}$ & -1.1804060 & -3.5023890 & -0.0768530 \\
\hline & $\mathrm{C}$ & -0.7281620 & -4.7846760 & -0.3815910 \\
\hline & $\mathrm{C}$ & 0.7281900 & -4.7847500 & -0.3814710 \\
\hline & $\mathrm{C}$ & 1.1804820 & -3.5024320 & -0.0767510 \\
\hline & $\mathrm{C}$ & -3.6229270 & -0.7286050 & 0.0838310 \\
\hline 8 & $\mathrm{C}$ & -2.4428590 & -2.7733950 & 0.0873720 \\
\hline 9 & $\mathrm{C}$ & -3.7798400 & -3.0222860 & 0.3913300 \\
\hline & $\mathrm{C}$ & -4.5075130 & -1.7607210 & 0.3898020 \\
\hline & $\mathrm{C}$ & 2.4429180 & -2.7734710 & 0.0878960 \\
\hline & $\mathrm{C}$ & 3.6229460 & -0.7286550 & 0.0844980 \\
\hline & $\mathrm{C}$ & 4.5075300 & -1.7607400 & 0.3906060 \\
\hline & $\mathrm{C}$ & 3.7799160 & -3.0223530 & 0.3919990 \\
\hline & $\mathrm{C}$ & 2.4428590 & 2.7733950 & -0.0873720 \\
\hline & $\mathrm{C}$ & -1.1804820 & 3.5024320 & 0.0767510 \\
\hline & $\mathrm{C}$ & -2.4429180 & 2.7734710 & -0.0878960 \\
\hline & $\mathrm{C}$ & -3.6229460 & 0.7286550 & -0.0844980 \\
\hline & $\mathrm{C}$ & -4.5075300 & 1.7607400 & -0.3906060 \\
\hline 0 & $\mathrm{C}$ & -3.7799160 & 3.0223530 & -0.3919990 \\
\hline & $\mathrm{C}$ & -0.7281900 & 4.7847500 & 0.3814710 \\
\hline & $\mathrm{C}$ & 0.7281620 & 4.7846760 & 0.3815910 \\
\hline 33 & $\mathrm{C}$ & 1.1804060 & 3.5023890 & 0.0768530 \\
\hline 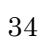 & $\mathrm{C}$ & 3.6229270 & 0.7286050 & -0.0838310 \\
\hline & $\mathrm{C}$ & 4.5075130 & 1.7607210 & -0.3898020 \\
\hline 5 & $\mathrm{C}$ & 3.7798400 & 3.0222860 & -0.3913300 \\
\hline 7 & $\mathrm{C}$ & -5.8713460 & 2.0176890 & -0.8536870 \\
\hline 8 & $\mathrm{C}$ & -5.9330170 & 3.4222090 & -1.0968560 \\
\hline 9 & $\mathrm{C}$ & -4.6867490 & 4.0734530 & -0.8548480 \\
\hline 0 & $\mathrm{C}$ & -4.6864820 & -4.0733500 & 0.8546630 \\
\hline 1 & $\mathrm{C}$ & -5.9328080 & -3.4221820 & 1.0965680 \\
\hline & $\mathrm{C}$ & -5.8712170 & -2.0176680 & 0.8533100 \\
\hline 43 & $\mathrm{C}$ & -1.1864250 & -6.0938340 & -0.8475780 \\
\hline & $\mathrm{C}$ & -0.0000870 & -6.8476040 & -1.0927650 \\
\hline 45 & $\mathrm{C}$ & 1.1863260 & -6.0939920 & -0.8474090 \\
\hline & $\mathrm{C}$ & 5.8713460 & -2.0176890 & 0.8536870 \\
\hline & $\mathrm{C}$ & 5.9330170 & -3.4222090 & 1.0968560 \\
\hline & $\mathrm{C}$ & 4.6867490 & -4.0734530 & 0.8548480 \\
\hline & $\mathrm{C}$ & 5.8712170 & 2.0176680 & -0.8533100 \\
\hline U & $\mathrm{C}$ & 5.9328080 & 3.4221820 & -1.0965680 \\
\hline
\end{tabular}

\begin{tabular}{|c|c|c|c|c|}
\hline 1 & $\mathrm{C}$ & 4.6864820 & 4.0733500 & 6630 \\
\hline 5 & $\mathrm{C}$ & -1.1863260 & 6.0939920 & 0.8474090 \\
\hline 53 & $\mathrm{C}$ & 0.0000870 & 6.8476040 & 1.0927650 \\
\hline & $\mathrm{C}$ & 1.1864250 & 6.0938340 & 0.8475780 \\
\hline & $\mathrm{C}$ & 6.9718100 & -1.2478860 & 1.1871470 \\
\hline & $\mathrm{C}$ & 8.1356730 & -1.8908040 & 1.6895920 \\
\hline & $\mathrm{C}$ & 8.1966670 & -3.2563350 & 1.8992270 \\
\hline & $\mathrm{C}$ & 7.0599540 & -4.0708930 & 1.6296860 \\
\hline & $\mathrm{C}$ & 6.9261540 & -5.4627160 & 1.8998400 \\
\hline & $\mathrm{C}$ & 5.7143630 & -6.0953660 & 1.6907630 \\
\hline & $\mathrm{C}$ & 4.5737790 & -5.4116120 & 1.1887020 \\
\hline & $\mathrm{C}$ & -0.0001470 & -8.1448740 & -1.6327120 \\
\hline & $\mathrm{C}$ & 1.2727710 & -8.7219930 & -1.9061740 \\
\hline & $\mathrm{C}$ & 2.4258420 & -7.9888420 & -1.6933510 \\
\hline & $\mathrm{C}$ & 2.4023840 & -6.6620050 & -1.1841050 \\
\hline & $\mathrm{C}$ & -2.4025300 & -6.6617500 & -1.1842400 \\
\hline & $\mathrm{C}$ & -2.4261200 & -7.9886030 & -1.6934110 \\
\hline 8 & $\mathrm{C}$ & -1.2731210 & -8.7218810 & -1.9061860 \\
\hline & $\mathrm{C}$ & -7.0596560 & -4.0708810 & 1.6295940 \\
\hline & $\mathrm{C}$ & -8.1963530 & -3.2563440 & 1.8992650 \\
\hline & $\mathrm{C}$ & -8.1353930 & -1.8908090 & 1.6896330 \\
\hline & $\mathrm{C}$ & -6.9715910 & -1.2478580 & 1.1870540 \\
\hline & $\mathrm{C}$ & -4.5733400 & -5.4114050 & 1.1888430 \\
\hline & $\mathrm{C}$ & -5.7138630 & -6.0952050 & 1.6909890 \\
\hline & $\mathrm{C}$ & -6.9257270 & -5.4626570 & 1.8999590 \\
\hline & $\mathrm{C}$ & -7.0599540 & 4.0708930 & -1.6296860 \\
\hline 7 & $\mathrm{C}$ & -6.9261540 & 5.4627160 & -1.8998400 \\
\hline & $\mathrm{C}$ & -5.7143630 & 6.0953660 & -1.6907630 \\
\hline 79 & $\mathrm{C}$ & -4.5737790 & 5.4116120 & -1.1887020 \\
\hline ) & $\mathrm{C}$ & -6.9718100 & 1.2478860 & -1.1871470 \\
\hline & $\mathrm{C}$ & -8.1356730 & 1.8908040 & -1.6895920 \\
\hline & $\mathrm{C}$ & -8.1966670 & 3.2563350 & -1.8992270 \\
\hline 8 & $\mathrm{C}$ & 0.0001470 & 8.1448740 & 1.6327120 \\
\hline & $\mathrm{C}$ & 1.2731210 & 8.7218810 & 1.9061860 \\
\hline 85 & $\mathrm{C}$ & 2.4261200 & 7.9886030 & 1.6934110 \\
\hline & $\mathrm{C}$ & 2.4025300 & 6.6617500 & 1.1842400 \\
\hline 87 & $\mathrm{C}$ & -2.4023840 & 6.6620050 & 1.1841050 \\
\hline & $\mathrm{C}$ & -2.4258420 & 7.9888420 & 1.6933510 \\
\hline 89 & $\mathrm{C}$ & -1.2727710 & 8.7219930 & 1.9061740 \\
\hline & $\mathrm{C}$ & 6.9715910 & 1.2478580 & -1.1870540 \\
\hline 91 & $\mathrm{C}$ & 8.1353930 & 1.8908090 & -1.6896330 \\
\hline & $\mathrm{C}$ & 8.1963530 & 3.2563440 & -1.8992650 \\
\hline 93 & $\mathrm{C}$ & 7.0596560 & 4.0708810 & -1.6295940 \\
\hline 94 & $\mathrm{C}$ & 6.9257270 & 5.4626570 & -1.8999590 \\
\hline & $\mathrm{C}$ & 5.7138630 & 6.0952050 & -1.6909890 \\
\hline 9 & $\mathrm{C}$ & 4.5733400 & 5.4114050 & -1.1888430 \\
\hline & $\mathrm{H}$ & 6.9612120 & -0.1674400 & 1.0965310 \\
\hline & $\mathrm{H}$ & 8.9991000 & -1.2770040 & 1.9322670 \\
\hline & $\mathrm{H}$ & 9.1006090 & -3.7082150 & 2.3001360 \\
\hline & $\mathrm{H}$ & 7.7707650 & -6.0175830 & 2.3008060 \\
\hline & $\mathrm{H}$ & 5.6169660 & -7.1501740 & 1.9337370 \\
\hline
\end{tabular}




$\begin{array}{lllll}102 & \mathrm{H} & 3.6341390 & -5.9451240 & 1.0987700 \\ 103 & \mathrm{H} & 1.3319010 & -9.7286690 & -2.3125540 \\ 104 & \mathrm{H} & 3.3884420 & -8.4294740 & -1.9390610 \\ 105 & \mathrm{H} & 3.3332430 & -6.1137620 & -1.0918880 \\ 106 & \mathrm{H} & -3.3333530 & -6.1134050 & -1.0921560 \\ 107 & \mathrm{H} & -3.3887720 & -8.4291270 & -1.9391090 \\ 108 & \mathrm{H} & -1.3323680 & -9.7285560 & -2.3125500 \\ 109 & \mathrm{H} & -9.1002110 & -3.7082460 & 2.3003360 \\ 110 & \mathrm{H} & -8.9988100 & -1.2770370 & 1.9324140 \\ 111 & \mathrm{H} & -6.9610590 & -0.1674280 & 1.0962450 \\ 112 & \mathrm{H} & -3.6336680 & -5.9448790 & 1.0988860 \\ 113 & \mathrm{H} & -5.6163480 & -7.1499660 & 1.9341150 \\ 114 & \mathrm{H} & -7.7702570 & -6.0175320 & 2.3010860 \\ 115 & \mathrm{H} & -7.7707650 & 6.0175830 & -2.3008060 \\ 116 & \mathrm{H} & -5.6169660 & 7.1501740 & -1.9337370 \\ 117 & \mathrm{H} & -3.6341390 & 5.9451240 & -1.0987700\end{array}$

$\begin{array}{lllll}118 & \mathrm{H} & -6.9612120 & 0.1674400 & -1.0965310 \\ 119 & \mathrm{H} & -8.9991000 & 1.2770040 & -1.9322670 \\ 120 & \mathrm{H} & -9.1006090 & 3.7082150 & -2.3001360 \\ 121 & \mathrm{H} & 1.3323680 & 9.7285560 & 2.3125500 \\ 122 & \mathrm{H} & 3.3887720 & 8.4291270 & 1.9391090 \\ 123 & \mathrm{H} & 3.3333530 & 6.1134050 & 1.0921560 \\ 124 & \mathrm{H} & -3.3332430 & 6.1137620 & 1.0918880 \\ 125 & \mathrm{H} & -3.3884420 & 8.4294740 & 1.9390610 \\ 126 & \mathrm{H} & -1.3319010 & 9.7286690 & 2.3125540 \\ 127 & \mathrm{H} & 6.9610590 & 0.1674280 & -1.0962450 \\ 128 & \mathrm{H} & 8.9988100 & 1.2770370 & -1.9324140 \\ 129 & \mathrm{H} & 9.1002110 & 3.7082460 & -2.3003360 \\ 130 & \mathrm{H} & 7.7702570 & 6.0175320 & -2.3010860 \\ 131 & \mathrm{H} & 5.6163480 & 7.1499660 & -1.9341150 \\ 132 & \mathrm{H} & 3.6336680 & 5.9448790 & -1.0988860\end{array}$

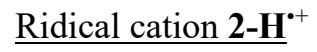

$\underline{\text { Total energy }=-3779.6904 \text { a.u., Imaginary frequency }=0}$

\begin{tabular}{|c|c|c|c|c|c|c|c|c|c|}
\hline 1 & $\mathrm{C}$ & 1.1931790 & 0.6889900 & 0.0221380 & 38 & $\mathrm{C}$ & -5.9341600 & 3.4232150 & -1.0834400 \\
\hline 2 & $\mathrm{C}$ & 0.0000000 & 1.3778330 & -0.0275630 & 39 & $\mathrm{C}$ & -4.6892940 & 4.0735860 & -0.8425810 \\
\hline 3 & $\mathrm{C}$ & -1.1931790 & 0.6889900 & 0.0221330 & 40 & $\mathrm{C}$ & -4.6892850 & -4.0735770 & 0.8426250 \\
\hline 4 & $\mathrm{C}$ & -1.1931790 & -0.6889900 & -0.0221380 & 41 & $\mathrm{C}$ & -5.9341520 & -3.4232040 & 1.0834770 \\
\hline 5 & $\mathrm{C}$ & 0.0000000 & -1.3778330 & 0.0275630 & 42 & $\mathrm{C}$ & -5.8729690 & -2.0203980 & 0.8408510 \\
\hline 6 & $\mathrm{C}$ & 1.1931790 & -0.6889900 & -0.0221330 & 43 & $\mathrm{C}$ & -1.1850220 & -6.0961770 & -0.8347210 \\
\hline 7 & $\mathrm{~N}$ & 2.3760850 & -1.3720720 & -0.0375130 & 44 & $\mathrm{C}$ & 0.0000050 & -6.8488090 & -1.0792490 \\
\hline 8 & $\mathrm{~N}$ & 2.3760860 & 1.3720730 & 0.0375190 & 45 & $\mathrm{C}$ & 1.1850290 & -6.0961830 & -0.8346900 \\
\hline 9 & $\mathrm{~N}$ & 0.0000000 & 2.7437910 & -0.0473840 & 46 & $\mathrm{C}$ & 5.8729720 & -2.0204040 & 0.8408380 \\
\hline 10 & $\mathrm{~N}$ & -2.3760850 & 1.3720720 & 0.0375130 & 47 & $\mathrm{C}$ & 5.9341600 & -3.4232150 & 1.0834400 \\
\hline 11 & $\mathrm{~N}$ & -2.3760860 & -1.3720730 & -0.0375190 & 48 & $\mathrm{C}$ & 4.6892940 & -4.0735860 & 0.8425810 \\
\hline 12 & $\mathrm{~N}$ & 0.0000000 & -2.7437910 & 0.0473840 & 49 & $\mathrm{C}$ & 5.8729690 & 2.0203980 & -0.8408510 \\
\hline 13 & $\mathrm{C}$ & -1.1818040 & -3.4961320 & -0.0726030 & 50 & $\mathrm{C}$ & 5.9341520 & 3.4232040 & -1.0834770 \\
\hline 14 & $\mathrm{C}$ & -0.7242700 & -4.7898310 & -0.3680400 & 51 & $\mathrm{C}$ & 4.6892850 & 4.0735770 & -0.8426250 \\
\hline 15 & $\mathrm{C}$ & 0.7242710 & -4.7898320 & -0.3680290 & 52 & $\mathrm{C}$ & -1.1850290 & 6.0961830 & 0.8346900 \\
\hline 16 & $\mathrm{C}$ & 1.1818040 & -3.4961310 & -0.0726000 & 53 & $\mathrm{C}$ & -0.0000050 & 6.8488090 & 1.0792490 \\
\hline 17 & $\mathrm{C}$ & -3.6183520 & -0.7243350 & 0.0808770 & 54 & $\mathrm{C}$ & 1.1850220 & 6.0961770 & 0.8347210 \\
\hline 18 & $\mathrm{C}$ & -2.4368350 & -2.7714530 & 0.0845270 & 55 & $\mathrm{C}$ & 6.9709970 & -1.2484360 & 1.1824580 \\
\hline 19 & $\mathrm{C}$ & -3.7862710 & -3.0215250 & 0.3794280 & 56 & $\mathrm{C}$ & 8.1323810 & -1.8885740 & 1.6896940 \\
\hline 20 & $\mathrm{C}$ & -4.5101550 & -1.7667720 & 0.3777410 & 57 & $\mathrm{C}$ & 8.1923660 & -3.2555480 & 1.8982610 \\
\hline 21 & $\mathrm{C}$ & 2.4368350 & -2.7714530 & 0.0845270 & 58 & $\mathrm{C}$ & 7.0580770 & -4.0703590 & 1.6230450 \\
\hline 22 & $\mathrm{C}$ & 3.6183530 & -0.7243350 & 0.0808800 & 59 & $\mathrm{C}$ & 6.9223490 & -5.4600900 & 1.8993180 \\
\hline 23 & $\mathrm{C}$ & 4.5101570 & -1.7667740 & 0.3777340 & 60 & $\mathrm{C}$ & 5.7095170 & -6.0938990 & 1.6917480 \\
\hline 24 & $\mathrm{C}$ & 3.7862740 & -3.0215270 & 0.3794100 & 61 & $\mathrm{C}$ & 4.5727600 & -5.4105710 & 1.1849600 \\
\hline 25 & $\mathrm{C}$ & 2.4368350 & 2.7714530 & -0.0845270 & 62 & $\mathrm{C}$ & 0.0000090 & -8.1426070 & -1.6261180 \\
\hline 26 & $\mathrm{C}$ & -1.1818040 & 3.4961310 & 0.0726000 & 63 & $\mathrm{C}$ & 1.2720450 & -8.7170920 & -1.9055700 \\
\hline 27 & $\mathrm{C}$ & -2.4368350 & 2.7714530 & -0.0845270 & 64 & $\mathrm{C}$ & 2.4267050 & -7.9837780 & -1.6940060 \\
\hline 28 & $\mathrm{C}$ & -3.6183530 & 0.7243350 & -0.0808800 & 65 & $\mathrm{C}$ & 2.4018220 & -6.6605500 & -1.1798790 \\
\hline 29 & $\mathrm{C}$ & -4.5101570 & 1.7667740 & -0.3777340 & 66 & $\mathrm{C}$ & -2.4018090 & -6.6605370 & -1.1799430 \\
\hline 30 & $\mathrm{C}$ & -3.7862740 & 3.0215270 & -0.3794100 & 67 & $\mathrm{C}$ & -2.4266850 & -7.9837650 & -1.6940710 \\
\hline 31 & $\mathrm{C}$ & -0.7242710 & 4.7898320 & 0.3680290 & 68 & $\mathrm{C}$ & -1.2720230 & -8.7170850 & -1.9056050 \\
\hline 32 & $\mathrm{C}$ & 0.7242700 & 4.7898310 & 0.3680400 & 69 & $\mathrm{C}$ & -7.0580640 & -4.0703410 & 1.6231010 \\
\hline 33 & $\mathrm{C}$ & 1.1818040 & 3.4961320 & 0.0726030 & 70 & $\mathrm{C}$ & -8.1923540 & -3.2555280 & 1.8983080 \\
\hline 34 & $\mathrm{C}$ & 3.6183520 & 0.7243350 & -0.0808770 & 71 & $\mathrm{C}$ & -8.1323730 & -1.8885570 & 1.6897180 \\
\hline 35 & $\mathrm{C}$ & 4.5101550 & 1.7667720 & -0.3777410 & 72 & $\mathrm{C}$ & -6.9709930 & -1.2484260 & 1.1824640 \\
\hline 36 & $\mathrm{C}$ & 3.7862710 & 3.0215250 & -0.3794280 & 73 & $\mathrm{C}$ & -4.5727450 & -5.4105540 & 1.1850340 \\
\hline 37 & $\mathrm{C}$ & -5.8729720 & 2.0204040 & -0.8408380 & 74 & $\mathrm{C}$ & -5.7094980 & -6.0938750 & 1.6918400 \\
\hline
\end{tabular}




$\begin{array}{ll}-5.4600670 & 1.8994010 \\ 4.0703590 & -1.6230450 \\ 5.4600900 & -1.8993180 \\ 6.0938990 & -1.6917480 \\ 8.1426070 & 1.6261180 \\ 8.7170850 & 1.9056050 \\ 7.9837650 & 1.6940710 \\ 6.6605370 & 1.1799430 \\ 6.6605500 & 1.1798790 \\ 7.9837780 & 1.6940060 \\ 8.7170920 & 1.9055700 \\ 1.2484260 & -1.1824640 \\ 1.8885570 & -1.6897180 \\ 3.2555280 & -1.8983080 \\ 4.0703410 & -1.6231010 \\ 5.4600670 & -1.8994010 \\ 6.0938750 & -1.6918400 \\ 5.4105540 & -1.1850340 \\ -0.1677390 & 1.0953350 \\ -1.2749830 & 1.9390740 \\ -3.7060340 & 2.3039090 \\ -6.0142000 & 2.3050040 \\ -7.1462790 & 1.9416880 \\ -5.9430870 & 1.0990180 \\ -9.7212770 & -2.3167960 \\ -8.4219620 & -1.9465830 \\ -6.1111850 & -1.0911900 \\ -6.1111680 & -1.0912790 \\ -8.4219440 & -1.9466750 \\ -9.7212700 & -2.3168320\end{array}$

$\begin{array}{lllll}79 & \mathrm{C} & -4.5727600 & 5.4105710 & -1.1849600 \\ 80 & \mathrm{C} & -6.9709970 & 1.2484360 & -1.1824580 \\ 81 & \mathrm{C} & -8.1323810 & 1.8885740 & -1.6896940 \\ 82 & \mathrm{C} & -8.1923660 & 3.2555480 & -1.8982610 \\ 109 & \mathrm{H} & -9.0943210 & -3.7060090 & 2.3039700 \\ 110 & \mathrm{H} & -8.9932330 & -1.2749640 & 1.9390920 \\ 111 & \mathrm{H} & -6.9594790 & -0.1677310 & 1.0953250 \\ 112 & \mathrm{H} & -3.6321740 & -5.9430680 & 1.0991010 \\ 113 & \mathrm{H} & -5.6105750 & -7.1462500 & 1.9418020 \\ 114 & \mathrm{H} & -7.7645910 & -6.0141710 & 2.3051020 \\ 115 & \mathrm{H} & -7.7646120 & 6.0142000 & -2.3050040 \\ 116 & \mathrm{H} & -5.6105990 & 7.1462790 & -1.9416880 \\ 117 & \mathrm{H} & -3.6321910 & 5.9430870 & -1.0990180 \\ 118 & \mathrm{H} & -6.9594810 & 0.1677390 & -1.0953350 \\ 119 & \mathrm{H} & -8.9932420 & 1.2749830 & -1.9390740 \\ 120 & \mathrm{H} & -9.0943370 & 3.7060340 & -2.3039090 \\ 121 & \mathrm{H} & 1.3316050 & 9.7212700 & 2.3168320 \\ 122 & \mathrm{H} & 3.3879010 & 8.4219440 & 1.9466750 \\ 123 & \mathrm{H} & 3.3323380 & 6.1111680 & 1.0912790 \\ 124 & \mathrm{H} & -3.3323510 & 6.1111850 & 1.0911900 \\ 125 & \mathrm{H} & -3.3879250 & 8.4219620 & 1.9465830 \\ 126 & \mathrm{H} & -1.3316330 & 9.7212770 & 2.3167960 \\ 127 & \mathrm{H} & 6.9594790 & 0.1677310 & -1.0953250 \\ 128 & \mathrm{H} & 8.9932330 & 1.2749640 & -1.9390920 \\ 129 & \mathrm{H} & 9.0943210 & 3.7060090 & -2.3039700 \\ 130 & \mathrm{H} & 7.7645910 & 6.0141710 & -2.3051020 \\ 131 & \mathrm{H} & 5.6105750 & 7.1462500 & -1.9418020 \\ 132 & \mathrm{H} & 3.6321740 & 5.9430680 & -1.0991010\end{array}$

Dication 2-H ${ }^{2+}$

$\underline{\text { Total energy }}=\underline{-3779.4193 \text { a.u., Imaginary frequency }=0}$

$\begin{array}{lllll}1 & \mathrm{C} & 1.1912800 & 0.6878860 & 0.0206590 \\ 2 & \mathrm{C} & -0.0000010 & 1.3756330 & -0.0262490 \\ 3 & \mathrm{C} & -1.1912810 & 0.6878870 & 0.0206680 \\ 4 & \mathrm{C} & -1.1912800 & -0.6878860 & -0.0206590 \\ 5 & \mathrm{C} & 0.0000010 & -1.3756330 & 0.0262490 \\ 6 & \mathrm{C} & 1.1912810 & -0.6878870 & -0.0206680 \\ 7 & \mathrm{~N} & 2.3722280 & -1.3698150 & -0.0271860 \\ 8 & \mathrm{~N} & 2.3722270 & 1.3698140 & 0.0271740 \\ 9 & \mathrm{~N} & -0.0000010 & 2.7393430 & -0.0371540 \\ 10 & \mathrm{~N} & -2.3722280 & 1.3698150 & 0.0271860 \\ 11 & \mathrm{~N} & -2.3722270 & -1.3698140 & -0.0271740 \\ 12 & \mathrm{~N} & 0.0000010 & -2.7393430 & 0.0371540 \\ 13 & \mathrm{C} & -1.1837090 & -3.4905030 & -0.0702400 \\ 14 & \mathrm{C} & -0.7214110 & -4.7954790 & -0.3578260 \\ 15 & \mathrm{C} & 0.7214090 & -4.7954810 & -0.3578260 \\ 16 & \mathrm{C} & 1.1837090 & -3.4905050 & -0.0702370 \\ 17 & \mathrm{C} & -3.6144520 & -0.7198860 & 0.0785000 \\ 18 & \mathrm{C} & -2.4310050 & -2.7702860 & 0.0821510 \\ 19 & \mathrm{C} & -3.7926010 & -3.0219100 & 0.3690740 \\ 20 & \mathrm{C} & -4.5136340 & -1.7721230 & 0.3674510 \\ 21 & \mathrm{C} & 2.4310080 & -2.7702880 & 0.0821460\end{array}$

$\begin{array}{lll}22 & \mathrm{C} & 3.6144520 \\ 23 & \mathrm{C} & 4.5136420 \\ 24 & \mathrm{C} & 3.7926060 \\ 25 & \mathrm{C} & 2.4310050 \\ 26 & \mathrm{C} & -1.1837090 \\ 27 & \mathrm{C} & -2.4310080 \\ 28 & \mathrm{C} & -3.6144520 \\ 29 & \mathrm{C} & -4.5136420 \\ 30 & \mathrm{C} & -3.7926060 \\ 31 & \mathrm{C} & -0.7214090 \\ 32 & \mathrm{C} & 0.7214110 \\ 33 & \mathrm{C} & 1.1837090 \\ 34 & \mathrm{C} & 3.6144520 \\ 35 & \mathrm{C} & 4.5136340 \\ 36 & \mathrm{C} & 3.7926010 \\ 37 & \mathrm{C} & -5.8735540 \\ 38 & \mathrm{C} & -5.9342120 \\ 39 & \mathrm{C} & -4.6910450 \\ 40 & \mathrm{C} & -4.6910390 \\ 41 & \mathrm{C} & -5.9342080 \\ 42 & \mathrm{C} & -5.8735330\end{array}$

$-0.7198850$

0.0785080

$-1.7721270$

0.3674240

$-3.0219130$

0.3690610

2.7702860

$-0.0821510$

3.4905050

0.0702370

2.7702880

0.7198850

$-0.0821460$

1.7721270

$-0.0785080$

3.0219130

$-0.3674240$

4.7954810

$-0.3690610$

4.7954790

0.3578260

3.4905030

0.3578260

0.7198860

0.0702400

1.7721230

$-0.0785000$

3.0219100

$-0.3674510$

$-0.3690740$

2.0222410

$-0.8321020$

3.4233440

$-1.0753630$

4.0732490

$-0.8338880$

$-4.0732490$

0.8338940

$-3.4233480$

1.0753650

$-2.0222340$

0.8321750 


\begin{tabular}{|c|c|c|c|c|c|c|c|c|c|}
\hline 43 & $\mathrm{C}$ & -1.1837930 & -6.0975200 & -0.8261010 & 88 & $\mathrm{C}$ & -2.4279600 & 7.9783610 & 1.6989580 \\
\hline 44 & $\mathrm{C}$ & -0.0000040 & -6.8488710 & -1.0712840 & 89 & $\mathrm{C}$ & -1.2708210 & 8.7116270 & 1.9108100 \\
\hline 45 & $\mathrm{C}$ & 1.1837880 & -6.0975200 & -0.8261140 & 90 & $\mathrm{C}$ & 6.9708300 & 1.2480890 & -1.1813930 \\
\hline 46 & $\mathrm{C}$ & 5.8735540 & -2.0222410 & 0.8321020 & 91 & $\mathrm{C}$ & 8.1284350 & 1.8849650 & -1.6945070 \\
\hline 47 & $\mathrm{C}$ & 5.9342120 & -3.4233440 & 1.0753630 & 92 & $\mathrm{C}$ & 8.1870890 & 3.2540590 & -1.9034170 \\
\hline 48 & $\mathrm{C}$ & 4.6910450 & -4.0732490 & 0.8338880 & 93 & $\mathrm{C}$ & 7.0551840 & 4.0688380 & -1.6222950 \\
\hline 49 & $\mathrm{C}$ & 5.8735330 & 2.0222340 & -0.8321750 & 94 & $\mathrm{C}$ & 6.9182020 & 5.4564680 & -1.9045450 \\
\hline 50 & $\mathrm{C}$ & 5.9342080 & 3.4233480 & -1.0753650 & 95 & $\mathrm{C}$ & 5.7041540 & 6.0924030 & -1.6966860 \\
\hline 51 & $\mathrm{C}$ & 4.6910390 & 4.0732490 & -0.8338940 & 96 & $\mathrm{C}$ & 4.5721590 & 5.4106560 & -1.1839670 \\
\hline 52 & $\mathrm{C}$ & -1.1837880 & 6.0975200 & 0.8261140 & 97 & $\mathrm{H}$ & 6.9592930 & -0.1672780 & 1.0955210 \\
\hline 53 & $\mathrm{C}$ & 0.0000040 & 6.8488710 & 1.0712840 & 98 & $\mathrm{H}$ & 8.9870900 & -1.2718240 & 1.9503670 \\
\hline 54 & $\mathrm{C}$ & 1.1837930 & 6.0975200 & 0.8261010 & 99 & $\mathrm{H}$ & 9.0869800 & -3.7029850 & 2.3145760 \\
\hline 55 & $\mathrm{C}$ & 6.9708670 & -1.2480960 & 1.1812700 & 100 & $\mathrm{H}$ & 7.7580530 & -6.0095540 & 2.3158130 \\
\hline 56 & $\mathrm{C}$ & 8.1284540 & -1.8849580 & 1.6944410 & 101 & $\mathrm{H}$ & 5.6044100 & -7.1425690 & 1.9533450 \\
\hline 57 & $\mathrm{C}$ & 8.1870900 & -3.2540430 & 1.9034220 & 102 & $\mathrm{H}$ & 3.6314140 & -5.9431140 & 1.0995040 \\
\hline 58 & $\mathrm{C}$ & 7.0551790 & -4.0688220 & 1.6223230 & 103 & $\mathrm{H}$ & 1.3307370 & -9.7132340 & -2.3274690 \\
\hline 59 & $\mathrm{C}$ & 6.9181860 & -5.4564410 & 1.9046220 & 104 & $\mathrm{H}$ & 3.3876730 & -8.4147760 & -1.9581100 \\
\hline 60 & $\mathrm{C}$ & 5.7041400 & -6.0923800 & 1.6967590 & 105 & $\mathrm{H}$ & 3.3327030 & -6.1105760 & -1.0916150 \\
\hline 61 & $\mathrm{C}$ & 4.5721600 & -5.4106490 & 1.1839840 & 106 & $\mathrm{H}$ & -3.3327130 & -6.1105810 & -1.0915660 \\
\hline 62 & $\mathrm{C}$ & -0.0000070 & -8.1392820 & -1.6254150 & 107 & $\mathrm{H}$ & -3.3876930 & -8.4147820 & -1.9580570 \\
\hline 63 & $\mathrm{C}$ & 1.2708210 & -8.7116270 & -1.9108100 & 108 & $\mathrm{H}$ & -1.3307610 & -9.7132370 & -2.3274470 \\
\hline 64 & $\mathrm{C}$ & 2.4279600 & -7.9783610 & -1.6989580 & 109 & $\mathrm{H}$ & -9.0869870 & -3.7030110 & 2.3145460 \\
\hline 65 & $\mathrm{C}$ & 2.4021130 & -6.6600790 & -1.1789300 & 110 & $\mathrm{H}$ & -8.9870710 & -1.2718350 & 1.9504440 \\
\hline 66 & $\mathrm{C}$ & -2.4021230 & -6.6600830 & -1.1788950 & 111 & $\mathrm{H}$ & -6.9592540 & -0.1672690 & 1.0956690 \\
\hline 67 & $\mathrm{C}$ & -2.4279760 & -7.9783650 & -1.6989220 & 112 & $\mathrm{H}$ & -3.6314180 & -5.9431280 & 1.0994650 \\
\hline 68 & $\mathrm{C}$ & -1.2708400 & -8.7116290 & -1.9107910 & 113 & $\mathrm{H}$ & -5.6044340 & -7.1426030 & 1.9532300 \\
\hline 69 & $\mathrm{C}$ & -7.0551840 & -4.0688380 & 1.6222950 & 114 & $\mathrm{H}$ & -7.7580770 & -6.0095910 & 2.3157060 \\
\hline 70 & $\mathrm{C}$ & -8.1870890 & -3.2540590 & 1.9034170 & 115 & $\mathrm{H}$ & -7.7580530 & 6.0095540 & -2.3158130 \\
\hline 71 & $\mathrm{C}$ & -8.1284350 & -1.8849650 & 1.6945070 & 116 & $\mathrm{H}$ & -5.6044100 & 7.1425690 & -1.9533450 \\
\hline 72 & $\mathrm{C}$ & -6.9708300 & -1.2480890 & 1.1813930 & 117 & $\mathrm{H}$ & -3.6314140 & 5.9431140 & -1.0995040 \\
\hline 73 & $\mathrm{C}$ & -4.5721590 & -5.4106560 & 1.1839670 & 118 & $\mathrm{H}$ & -6.9592930 & 0.1672780 & -1.0955210 \\
\hline 74 & $\mathrm{C}$ & -5.7041540 & -6.0924030 & 1.6966860 & 119 & $\mathrm{H}$ & -8.9870900 & 1.2718240 & -1.9503670 \\
\hline 75 & $\mathrm{C}$ & -6.9182020 & -5.4564680 & 1.9045450 & 120 & $\mathrm{H}$ & -9.0869800 & 3.7029850 & -2.3145760 \\
\hline 76 & $\mathrm{C}$ & -7.0551790 & 4.0688220 & -1.6223230 & 121 & $\mathrm{H}$ & 1.3307610 & 9.7132370 & 2.3274470 \\
\hline 77 & $\mathrm{C}$ & -6.9181860 & 5.4564410 & -1.9046220 & 122 & $\mathrm{H}$ & 3.3876930 & 8.4147820 & 1.9580570 \\
\hline 78 & $\mathrm{C}$ & -5.7041400 & 6.0923800 & -1.6967590 & 123 & $\mathrm{H}$ & 3.3327130 & 6.1105810 & 1.0915660 \\
\hline 79 & $\mathrm{C}$ & -4.5721600 & 5.4106490 & -1.1839840 & 124 & $\mathrm{H}$ & -3.3327030 & 6.1105760 & 1.0916150 \\
\hline 80 & $\mathrm{C}$ & -6.9708670 & 1.2480960 & -1.1812700 & 125 & $\mathrm{H}$ & -3.3876730 & 8.4147760 & 1.9581100 \\
\hline 81 & $\mathrm{C}$ & -8.1284540 & 1.8849580 & -1.6944410 & 126 & $\mathrm{H}$ & -1.3307370 & 9.7132340 & 2.3274690 \\
\hline 82 & $\mathrm{C}$ & -8.1870900 & 3.2540430 & -1.9034220 & 127 & $\mathrm{H}$ & 6.9592540 & 0.1672690 & -1.0956690 \\
\hline 83 & $\mathrm{C}$ & 0.0000070 & 8.1392820 & 1.6254150 & 128 & $\mathrm{H}$ & 8.9870710 & 1.2718350 & -1.9504440 \\
\hline 84 & $\mathrm{C}$ & 1.2708400 & 8.7116290 & 1.9107910 & 129 & $\mathrm{H}$ & 9.0869870 & 3.7030110 & -2.3145460 \\
\hline 85 & $\mathrm{C}$ & 2.4279760 & 7.9783650 & 1.6989220 & 130 & $\mathrm{H}$ & 7.7580770 & 6.0095910 & -2.3157060 \\
\hline 86 & $\mathrm{C}$ & 2.4021230 & 6.6600830 & 1.1788950 & 131 & $\mathrm{H}$ & 5.6044340 & 7.1426030 & -1.9532300 \\
\hline 87 & $\mathrm{C}$ & -2.4021130 & 6.6600790 & 1.1789300 & 132 & $\mathrm{H}$ & 3.6314180 & 5.9431280 & -1.0994650 \\
\hline
\end{tabular}




\section{References}

[S1] SHELXT: G. M. Sheldrick, Acta Crystallogr. 2014, A70, C1437.

[S2] SIR2004: M. C. Burla, R. Caliandro, M. Camalli, B. Carrozzini, G. L. Cascarano, L. De Caro, C. Giacovazzo, G. Polidori, and R. Spagna, J. Appl. Crystllogr., 2005, 38, 381.

[S3] SHELXD97: G. M. Sheldrick, Acta Crystallography. 2008, A64, 112.

[S4] SHELXL (2016): G. M. Sheldrick, Acta Crystallogr. 2008, A64, 112.

[S5] Gaussian 09, Revision D.01, Frisch, M. J.; Trucks, G. W.; Schlegel, H. B.; Scuseria, G. E.; Robb, M. A.; Cheeseman, J. R.; Scalmani, G.; Barone, V.; Mennucci, B.; Petersson, G. A.; Nakatsuji, H.; Caricato, M.; Li, X.; Hratchian, H. P.; Izmaylov, A. F.; Bloino, J.; Zheng, G.; Sonnenberg, J. L.; Hada, M.; Ehara, M.; Toyota, K.; Fukuda, R.; Hasegawa, J.; Ishida, M.; Nakajima, T.; Honda, Y.; Kitao, O.; Nakai, H.; Vreven, T.; Montgomery, Jr.J. A.; Peralta, J. E.; Ogliaro, F.; Bearpark, M.; Heyd, J. J.; Brothers, E.; Kudin, K. N.; Staroverov, V. N.; Keith, T.; Kobayashi, R.; Normand, J.; Raghavachari, K.; Rendell, A.; Burant, J. C.; Iyengar, S. S.; Tomasi, J.; Cossi, M.; Rega, N.; Millam, J. M.; Klene, M.; Knox, J. E.; Cross, J. B.; Bakken, V.; Adamo, C.; Jaramillo, J.; Gomperts, R.; Stratmann, R. E.; Yazyev, O.; Austin, A. J.; Cammi, R.; Pomelli, C.; Ochterski, J. W.; Martin, R. L.; Morokuma, K.; Zakrzewski, V. G.; Voth, G. A.; Salvador, P.; Dannenberg, J. J.; Dapprich, S.; Daniels, A. D.; Farkas, O.; Foresman, J. B.; Ortiz, J. V.; Cioslowski, J. and Fox, D. J. Gaussian, Inc., Wallingford CT, 2013. 